\title{
Bis-Phosphonate Polymeric Ligands on Inorganic Nanoparticles
}

Supporting Information -

Authors: Karan Malhotra, Richard Fuku, Tsz Shan Chan, Nicole Kraljevic, Abootaleb Sedighi, Paul A. E. Piunno, and Ulrich J. Krull*

Affiliation:

Chemical Sensors Group, Department of Chemical and Physical Sciences, University of Toronto Mississauga, 3359 Mississauga Road North, Mississauga, Ontario, Canada, L5L 1C6.

* corresponding author; Email: ulrich.krull@utoronto.ca 


\section{Materials}

Multicolour quantum dots, nanoparticles and nanourchins were from Cytodiagnostics (Burlington, ON, Canada). Capped alkyl groups, oleic acid, were placed on $\mathrm{CdSe}_{1-x} \mathrm{~S}_{\mathrm{x}} / \mathrm{ZnS}$ (core/shell) with peak photoluminescence (PL) at $525 \mathrm{~nm}, 575 \mathrm{~nm}$, and $630 \mathrm{~nm}$. Iron oxide nanoparticles (IONPs) were capped oleic acid, and the gold nanourchins (AuNUs) were $50 \mathrm{~nm}, 60$ $\mathrm{nm}, 80 \mathrm{~nm}$, and $100 \mathrm{~nm}$. Cy3-DBCO (cyanine 3, dibenzocycloooctyne) was from Lumiprobe (Hunt Valley, Maryland, USA), and was dissolved in DMSO (dimethylsulfoxide) at a concentration of $1 \mathrm{~g} / \mathrm{L}$. DPS (4,4' - (1,2-ethenediyl)bis-1,1'-biphenyl) was from Alfa Aesar (Ward Hill, MA, USA) and was dissolved in dioxane to yield a saturated solution. Fluorescein reference dye was obtained from Invitrogen Thermo Fisher Scientific (Waltham, MA, USA) as a 1 mM solution in DMSO. 1Boc-ethylenediamine (98 \%) was from Combi-blocks (San Diego, CA, USA). Diethyl vinylphosphonate (97\%) was from TCI America (Portland, OR, USA). Deuterated DMSO$d 6$ and deuterated chloroform $\left(\mathrm{CDCl}_{3}\right)$ was from Cambridge Isotope Laboratories (Cambridge, MA). All other chemicals and solvents were from Sigma-Aldrich (Oakville, ON, Canada), Caledon Laboratories Ltd. (Georgetown, ON, Canada) and Fisher Scientific (Oakville, ON, Canada). ACS Reagent grade chemicals were used for all solvents unless otherwise stated. Buffer solutions were autoclaved prior to use and prepared using a Millipore water (MQ) purification system (Milli-Q, $18 \mathrm{M} \Omega \mathrm{cm}^{-1}$; Milipore Corp., Mississauga, ON, Canada). The following solutions were prepared for investigations related to the colloidal stability of polymer coated nanoparticles: $1 x$ PBS buffer, 5x PBS buffer, $1 \mathrm{M} \mathrm{NaCl}$, autoclaved MQ water, and Mouse Serum. 


\section{Instrumentation and Characterization Techniques}

NPs were characterized for their structural and colloidal properties using dynamic light scattering (DLS), ultraviolet-visible spectroscopy (UV-vis), transmission electron microscopy (TEM), and luminescence/lifetime spectroscopy. For QDs, the extinction coefficients at the first exciton peak were obtained from literature (summarized in Table S1) [1]. Spectral properties of Cy3-DBCO dye were obtained from Lumiprobe methods, and the data includes excitation maximum at $555 \mathrm{~nm}, \varepsilon(\lambda=555 \mathrm{~nm}) 150000 \mathrm{~L} \mathrm{~mol}^{-1} \mathrm{~cm}^{-1}$, emission maximum at $570 \mathrm{~nm}$, and fluorescence quantum yield ( $Q Y, \Phi)$ of $0.31 .^{a}$

Table S1 Extinction Coefficients for Alloyed Semiconductor Nanocrystals CdSeS/ZnS from Ref [1]

\begin{tabular}{|l|l|l|}
\hline Quantum Dots & $\lambda$ at first exciton peak $(\mathrm{nm})$ & $\varepsilon$ at first exciton peak $\left(\mathrm{M}^{-1} \mathrm{~cm}^{-1}\right)$ \\
\hline QD525 & 504 & $2.1 \pm 0.1 \times 10^{5}$ \\
\hline QD575 & 552 & $2.2 \pm 0.2 \times 10^{5}$ \\
\hline QD630 & 612 & $2.2 \pm 0.2 \times 10^{5}$ \\
\hline
\end{tabular}

The DLS measurements for gold nanoparticles were done using Malvern Zetasizer NanoZS with the following software parameters; sample refractive index of 1.496 , viscosity of $0.5900 \mathrm{cP}$, refractive index of 1.496 , and absorbance of 0.00 for Milli-Q water and Toluene at $25^{\circ} \mathrm{C}$. All measurements for hydrodynamic diameter were made in triplicate using polystyrene (PS) disposable cuvettes and quartz cuvettes.

UV-vis absorption spectroscopy of the nanoparticles was done using a VWR UV-vis spectrophotometer UV-1600PC (Mississauga, ON, Canada) with Milli-Q water as the blank solution.

a Cy3-DBCO spectral properties URL: https://www.lumiprobe.com/p/cy3-dbco 
Steady-state luminescence measurements were made using QuantaMaster Photon Technology International spectrofluorometer (London, ON, Canada). The excitation source, used for steady-state measurements was a xenon arc lamp (Ushio, Cypress, CA, USA) and the detector was a red-sensitive R928P photomultiplier tube (PMT, Hamamatsu, Bridgewater, NJ).

Luminescence lifetime measurements were made using a nitrogen-dye laser (via the stroboscopic method). The lasing dye used in these measurements was DPS $\left(4,4^{\prime}-(1,2-\right.$ ethenediyl)bis-1,1'-biphenyl) dissolved in dioxane at saturating conditions. An excitation wavelength of $402 \mathrm{~nm}$ was used and lifetime of the green QDs was measured at $530 \mathrm{~nm} .^{\mathrm{b}}$

Images related to the luminescence of QDs in gel electrophoresis experiments were captured using a BioRad Molecular Imager ${ }^{\circledR}$ GeIDoc $^{T M}$ XR (BioRad, Hercules, CA, USA). Additional images associated with ligand exchange and colloidal stability were obtained using an iPhone 8 (Apple, Cupertino, CA, USA).

UV-vis absorption and luminescence plate reader measurements were obtained from the BioTek Cytation 3 (Winooski, VT, USA) microplate reader. Images from the gel experiments, colloidal stability, and TEM were processed using ImageJ software (version 1.49u, National Institute of Health, USA) for additional clarity and NP size determination.

Aqueous samples for EM analysis were prepared by pre-treating carbon support grids (Product \# 01824 Ted Pella) with $1 \%$ acetic acid solution for 10 minutes. Excess solution was removed using a Kimwipe before adding of $7 \mu \mathrm{L}$ of QDs solution in water. The grids were

\footnotetext{
${ }^{\boldsymbol{b}}$ Note: The software used for all solution phase luminescence and lifetime measurements was FelixGX. The PMT response was adjusted across the spectral range using a response curve supplied by the manufacturer.
} 
incubated with the solution until dry. TEM images were obtained from a Talos L120C Transmission Electron Microscope (Thermo Fisher Scientific, Waltham, Mass, USA), and the instrument was located at the Microscopy Imaging Laboratory at the University of Toronto, Faculty of Medicine (Toronto, ON, Canada).

${ }^{1} \mathrm{H}$ NMR $(400 \mathrm{MHz}),{ }^{13} \mathrm{C}$ NMR $(101 \mathrm{MHz})$, and ${ }^{31} \mathrm{P}$ NMR $(162 \mathrm{MHz})$ spectra were recorded on a Bruker NMR spectrometer (Billerica, Mass, USA). $\mathrm{CDCl}_{3}$ and DMSO-d6 were used as solvents to prepare all NMR samples. Peak multiplicities were recorded using the following abbreviations: $\mathrm{s}$, singlet; bs, broad singlet; $\mathrm{d}$, doublet; $\mathrm{t}$, triplet; $\mathrm{m}$, multiplet, and the J coupling constant in $\mathrm{Hz}$.

Fourier transform infrared spectroscopy was done using a Thermo Fisher Scientific Nicolet iS20 spectrometer with the ATR attachment (Thermo Fisher Scientific, Waltham, Mass, USA). Spectra were collected using the OMNIC 9.9.549 software package.

The reverse-phase chromatography experiments were done using a BEH C-18 column (Waters, Milford, USA). The mass spectrometric data was acquired in the MSE mode using a quadrupole-time of flight LC-MS/MS instrument (XEVO G2-XS Q Tof, Waters). The collected MSMS data was processed in UNIFI software (Waters, Milford, USA).

\section{Experimental Methods}

Methoxy terminated polyethylene glycol derivatives (mPEG; MW of $750 \mathrm{~g} / \mathrm{mol}$ ) were synthesized from the mPEG-OH starting material using modified synthetic protocols based on reports previously presented in literature [2-4]. Other, bifunctional PEG derivatives (MW of $600 \mathrm{~g} / \mathrm{mol}$ ) were synthesized using HO-PEG-OH as a starting material, following established synthetic protocols [5]. Note that characterization data for the following reactions are presented in the appendix. 
3.1. Synthesis of amine functionalized mono-phosphonate ester anchoring group via a Michael type addition reaction

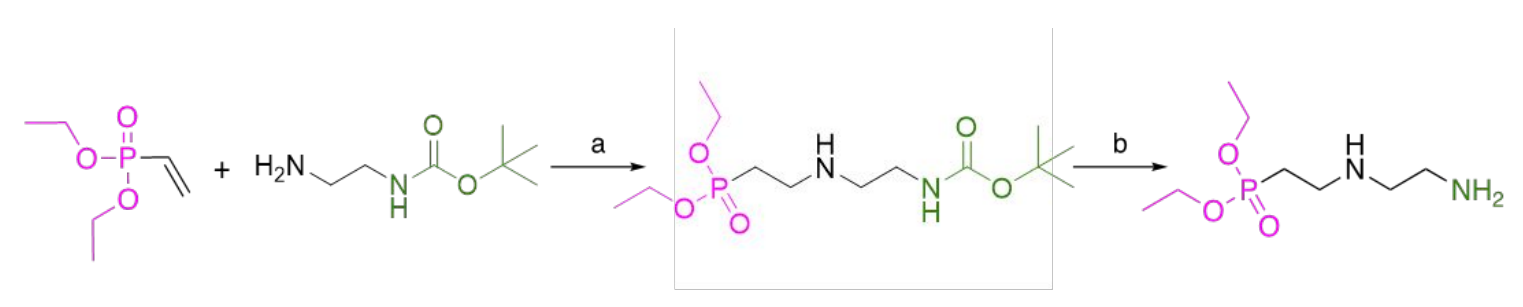

Scheme 1. Michael addition of vinyl phosphonate groups to 1-Boc-ethylenediamine.

\section{1a. Synthesis of mono-phosphonate-NHBoc [MPAOEt-NHBoc]}

$0.50 \mathrm{~g}(494 \mu \mathrm{L})$ of 1-Boc-ethylenediamine $(1 \mathrm{eq})$ and $0.54 \mathrm{~g}(505 \mu \mathrm{L})$ of diethyl vinylphosphonate $(1.1 \mathrm{eq})$ were added to $8 \mathrm{~mL} \mathrm{DI}$ water under an inert nitrogen atmosphere. The reaction was heated to $60^{\circ} \mathrm{C}$ and stirred over 24 hours. After heating, the solvent was removed under vacuum by rotary distillation (using toluene as an azeotrope for water). The crude product was obtained and purified by silica gel column chromatography $\left(10: 1 \mathrm{CHCl}_{3}: \mathrm{MeOH}\right)$ to yield a pale-yellow oil. (666 mg, Yield $66 \%$ ).

${ }^{1} \mathrm{H}$ NMR $(400 \mathrm{MHz}$, Chloroform-d) $\delta 5.05(\mathrm{~s}, 1 \mathrm{H}), 4.22-4.04(\mathrm{~m}, 4 \mathrm{H}), 3.24(\mathrm{q}, J=5.8 \mathrm{~Hz}$, 2H), $2.93(\mathrm{dt}, J=16.3,7.1 \mathrm{~Hz}, 2 \mathrm{H}), 2.75(\mathrm{t}, J=5.8 \mathrm{~Hz}, 2 \mathrm{H}), 1.98(\mathrm{dt}, J=18.2,7.1 \mathrm{~Hz}, 2 \mathrm{H}), 1.46(\mathrm{~s}, 9 \mathrm{H})$, $1.35(t, J=7.1 \mathrm{~Hz}, 6 \mathrm{H}) .{ }^{31} \mathrm{P}$ NMR $\left(162 \mathrm{MHz}\right.$, Chloroform-d) $\delta 30.53 .{ }^{13} \mathrm{C}$ NMR $(101 \mathrm{MHz}$, Chloroformd) $\delta 156.09,61.67,61.60,58.42,48.63,42.94,42.90,28.43,27.21,25.82,18.45,16.50,16.45$. LCMS-MS $(\mathrm{m} / \mathrm{z}$ for $[\mathrm{C} 13 \mathrm{H} 29 \mathrm{~N} 2 \mathrm{O} 5 \mathrm{P}])=325.19$, theoretical $=324.18$. $\mathrm{LC}$ conditions: Mobile phase consisted of a gradient of acetonitrile ( $5 \%-95 \%$ of $\mathrm{CH}_{3} \mathrm{CN}+0.1 \%$ formic acid from $0-3$ minutes) in $\mathrm{H}_{2} \mathrm{O}+0.1 \%$ formic acid. Retention time for MPAOEt-NHBoc in BEH C-18 column for LC-MS/MS was 4.48 minutes. 


\section{1b. Synthesis of mono-phosphonate amine, Boc deprotected [MPAOEt- $\mathrm{NH}_{2}$ ]}

$1 \mathrm{~mL}$ of anhydrous DCM was added to the $666 \mathrm{mg}$ of MPAOEt-NHBoc in a scintillation vial.

Next, $1 \mathrm{~mL}$ dry TFA was added dropwise and the reaction mixture was stirred for a further 12 hours. Separately, $40 \mathrm{~g}$ of Amberlite (IRN-78, ion exchange resin, nuclear grade) was stirred in a solution of $\mathrm{MeOH}$ for 30 minutes followed by transfer of the amberlite into a column with a fritted disk (column diameter was 1"). The reaction mixture was concentrated in vacuo and the remaining residue was transferred to the amberlite column for removal of TFA. The product was eluted in $\mathrm{MeOH}$ and the obtained residue was reduced in vacuo to obtain a clear yellow oil (622 mg, Yield $75 \%)$.

${ }^{1} \mathrm{H}$ NMR $(400 \mathrm{MHz}$, Chloroform-d) $\delta 4.23-4.04(\mathrm{~m}, 4 \mathrm{H}), 2.94(\mathrm{~m}, 2 \mathrm{H}), 2.82(\mathrm{~m}, 2 \mathrm{H}), 2.70$ $(\mathrm{m}, 2 \mathrm{H}), 2.00(\mathrm{~m}, 2 \mathrm{H}), 1.35(\mathrm{~m}, 6 \mathrm{H}), 1.28(\mathrm{~s}, 1 \mathrm{H}), 0.94-0.83(\mathrm{~m}, 1 \mathrm{H}), 0.09(\mathrm{~s}, 1 \mathrm{H}) .{ }^{31} \mathrm{P}$ NMR $(162$ $\mathrm{MHz}$, Chloroform-d) $\delta$ 30.62. ${ }^{13} \mathrm{C}$ NMR (101 MHz, Chloroform-d) $\delta 61.62,61.56,52.12,43.24$, $43.21,41.65,27.33,25.95,16.50,16.44$. LC-MS-MS $(\mathrm{m} / \mathrm{z}$ for $[\mathrm{C} 8 \mathrm{H} 21 \mathrm{~N} 2 \mathrm{O} 3 \mathrm{P}])=225.14$, theoretical $=224.24$. LC conditions: Mobile phase consisted of a gradient of acetonitrile $\left(5 \%-95 \%\right.$ of $\mathrm{CH}_{3} \mathrm{CN}$ $+0.1 \%$ formic acid from $0-3$ minutes) in $\mathrm{H}_{2} \mathrm{O}+0.1 \%$ formic acid. Retention time for MPAOEt$\mathrm{NH}_{2}$ in BEH C-18 column for LC-MS/MS was 1.12 minutes. 
3.2. Synthesis of amine functionalized bis-phosphonate ester anchoring group via a Michael type addition reaction

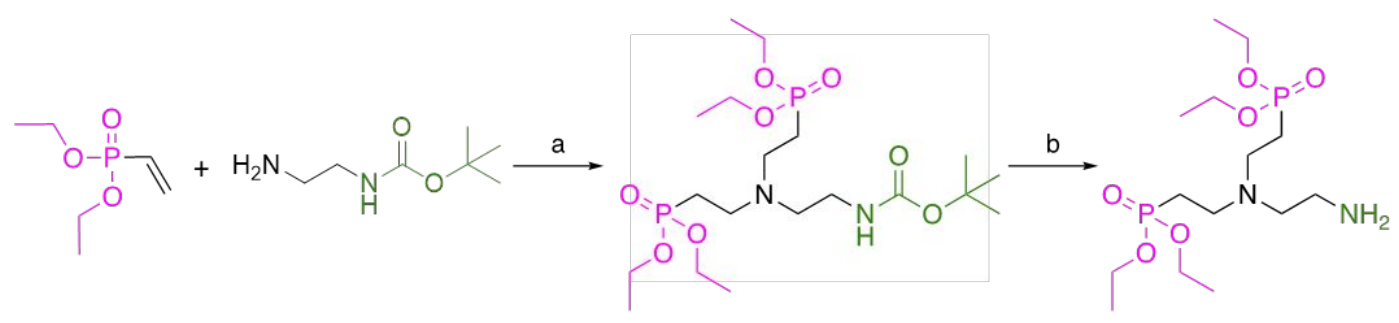

Scheme 2. Michael addition of vinyl phosphonate groups to 1-Boc-ethylenediamine.

\section{2a Synthesis of bis-phosphonate-NHBoc [BPAOEt-NHBoc]}

$0.50 \mathrm{~g}(494 \mu \mathrm{L})$ of 1-Boc-ethylenediamine $(1 \mathrm{eq})$ and $1.08 \mathrm{~g}(1.01 \mathrm{~mL})$ of diethyl vinylphosphonate ( $2.1 \mathrm{eq}$ ) were added to $8 \mathrm{~mL} \mathrm{DI}$ water under an inert nitrogen atmosphere. The reaction was heated to $60^{\circ} \mathrm{C}$ and stirred over 48 hours. After heating, the solvent was removed under vacuum by rotary distillation (using toluene as an azeotrope for water). The crude product was obtained and purified using a silica gel column chromatography (column diameter $1 \frac{1}{2}$ " with $70 \mathrm{~g}$ of silica gel 35-70 micron particle size; $10: 1 \mathrm{CH}_{2} \mathrm{Cl}_{2}: \mathrm{MeOH}$ ) to yield a clear pale-yellow oil. $(1.17 \mathrm{~g}$, Yield $78 \%)$.

${ }^{1} \mathrm{H}$ NMR $(400 \mathrm{MHz}$, Chloroform-d) $\delta 6.00(\mathrm{~s}, 1 \mathrm{H}), 4.26-4.07(\mathrm{~m}, 8 \mathrm{H}), 3.55-3.46(\mathrm{~m}, 2 \mathrm{H})$, $3.32(\mathrm{q}, J=7.5 \mathrm{~Hz}, 4 \mathrm{H}), 3.19(\mathrm{t}, J=5.7 \mathrm{~Hz}, 2 \mathrm{H}), 2.32-2.17(\mathrm{~m}, 4 \mathrm{H}), 1.45(\mathrm{~s}, 9 \mathrm{H}), 1.37(\mathrm{t}, J=7.1 \mathrm{~Hz}$, 12H). ${ }^{31} \mathrm{P}$ NMR (162 MHz, Chloroform-d) $\delta$ 30.65. ${ }^{13} \mathrm{C}$ NMR (101 MHz, Chloroform-d) $\delta$ 156.21, $80.08,62.72,62.66,52.46,48.05,36.08,28.23,21.61,20.20,16.34,16.28$. LC-MS-MS (m/z for $[\mathrm{C} 19 \mathrm{H} 42 \mathrm{~N} 2 \mathrm{O} 8 \mathrm{P} 2])=489.25$, theoretical $=488.50 . \mathrm{LC}$ conditions: Mobile phase consisted of a gradient of acetonitrile ( $5 \%-95 \%$ of $\mathrm{CH}_{3} \mathrm{CN}+0.1 \%$ formic acid from $0-3$ minutes) in $\mathrm{H}_{2} \mathrm{O}+0.1 \%$ formic acid. Retention time for BPAOEt-NHBoc was 6.30 minutes. 


\section{2b Synthesis of bis-phosphonate amine, Boc deprotected [BPAOEt- $\mathrm{NH}_{2}$ ]}

$1 \mathrm{~mL}$ of anhydrous DCM was added to the BPAOEt-NH $\mathrm{NH}_{2}$ a scintillation vial. Next, $1 \mathrm{~mL}$ of dry TFA was added dropwise and the reaction mixture, which was stirred for a further 12 hours. Separately, $40 \mathrm{~g}$ of Amberlite (IRN-78, ion exchange resin, nuclear grade) was stirred in a solution of $\mathrm{MeOH}$ for 30 minutes followed by transfer of the amberlite into a column with a fritted disk (column diameter was 1"). The reaction mixture was concentrated in vacuo and the remaining residue was transferred to the amberlite column for removal of TFA. The product was eluted in $\mathrm{MeOH}$ and the obtained residue was reduced in vacuo to obtain a clear yellow oil $(622 \mathrm{mg}$, Yield $75 \%)$.

${ }^{1} \mathrm{H}$ NMR (400 MHz, Chloroform-d) $\delta 4.20-4.01(\mathrm{~m}, 8 \mathrm{H}), 2.86-2.72(\mathrm{~m}, 6 \mathrm{H}), 2.54-2.46(\mathrm{~m}$, 2H), $2.00-1.86(m, 4 H), 1.34(t, J=7.1 \mathrm{~Hz}, 12 \mathrm{H}) .{ }^{31} \mathrm{P}$ NMR $\left(162 \mathrm{MHz}\right.$, Chloroform-d) $\delta 30.55 .{ }^{13} \mathrm{C}$ NMR $(101 \mathrm{MHz}$, Chloroform-d) $\delta 61.60,61.54,55.86,46.54,39.50,24.05,22.68,16.49,16.43$. LCMS-MS (m/z for [C14H34N2O6P2]) = 389.20, theoretical = 389.38. LC conditions: Mobile phase consisted of a gradient of acetonitrile ( $5 \%-95 \%$ of $\mathrm{CH}_{3} \mathrm{CN}+0.1 \%$ formic acid from $0-3$ minutes) in $\mathrm{H}_{2} \mathrm{O}+0.1 \%$ formic acid. Retention time for BPAOEt- $\mathrm{NH}_{2}$ was 5.47 minutes. 


\subsection{Synthesis of M/BPAPH-PIMA-PEG Polymers}

The synthesis of phosphonic acid-based PIMA polymers was realized via a two-step strategy summarized in Figure 3 of the main manuscript. Here, mPEG-NH${ }_{2}, \mathrm{M} / \mathrm{BPAOEt}-\mathrm{NH}_{2}$, and $\mathrm{N}_{3}$-PEG-NH $\mathrm{N}_{2}$ were reacted in various quantities with PIMA to yield the final PAOH-PIMA-PEG copolymers.

3.3a General Ligand Synthesis of Phosphonic Acid:mPEG-NH $\mathrm{N}_{2}$ (50:50 or 25:75), or Phosphonic Acid:mPEG-NH ${ }_{2}: \mathrm{N}_{3}-\mathrm{PEG}-\mathrm{NH}_{2}(50: 45: 5) .{ }^{c}$

Briefly, poly(isobutylene-alt-maleic anhydride) (0.25 g, $1.625 \mathrm{mmol}$ monomer units) was dissolved using $2 \mathrm{~mL}$ DMF in a $25 \mathrm{~mL}$ round bottom flask equipped with a magnetic stirring bar. The solution was subsequently heated to $65^{\circ} \mathrm{C}$. Separately, BPAOEt- $\mathrm{NH}_{2}$ (315 mg, $0.8125 \mathrm{mmol}$ ) and $\mathrm{NH}_{2}-\mathrm{PEG}-\mathrm{OCH}_{3}(610 \mathrm{mg}, 0.8125 \mathrm{mmol})$ were mixed in a vial with $1 \mathrm{~mL}$ of DMF. The contents of the vial were added dropwise to the PIMA solution and the reaction mixture was further stirred overnight at $60^{\circ} \mathrm{C}$. After removal of the solvent under vacuum for 24 hours, the residue was purified by silica gel column chromatography (column diameter 1 1 1/2" with $100 \mathrm{~g}$ of silica gel 3570 micron particle size; mobile phase: $100 \% \mathrm{CHCl}_{3}$ to $70: 30 \mathrm{CHCl}_{3}$ : $\mathrm{MeOH}$ ) to yield Bis-PAOEtPIMA-PEG as a pale-yellow oil (738 mg, Yield $65 \%$ ). NMR analysis of the synthesized polymer found a grafting ratio of $46 \%$ for bis-phosphonate amine and $54 \% \mathrm{mPEG}-\mathrm{NH}_{2}$ (MW of $750 \mathrm{~g} / \mathrm{mol}$ ). The column purified product was dissolved into a $25 \mathrm{~mL}$ round bottom flask, with $5 \mathrm{~mL}$ of $\mathrm{DCM}$, and placed in an ice bath $\left(0^{\circ} \mathrm{C}\right) . \mathrm{TMSBr}(8.125 \mathrm{mmol}, 0.873 \mathrm{~mL})$ was added dropwise to the

\footnotetext{
${ }^{\mathrm{C}}$ The following example is for the synthesis of BPAOEt-PIMA-PEG however, the general protocol is valid for the entire series of mono- or bis- polymers.
} 
reaction mixture and the solution was brought to room temperature overnight. Subsequently, the solvent was removed under reduced pressure, followed by addition of $5 \mathrm{~mL}$ of $\mathrm{MeOH}$. The reaction mixture was stirred for a further 8 hours. The solvent was again removed under reduced pressure and the resulting residue was redissolved in $\mathrm{MeOH}$. The mixture was vortexed, and the solvent was removed again via rotovap (this step was repeated for a total of three times). Finally, the resulting residue was concentrated via rotovap to yield a yellow oil (700 mg, yield $100 \%$ ).

Table S2 Summary of the Reaction Yields for the Synthesis of PA-PIMA-mPEG Polymers ${ }^{d}$

\begin{tabular}{|c|c|c|c|}
\hline \multirow{2}{*}{$\begin{array}{c}\text { Grafting Ratio of PA: } \\
\text { PEG / PA:PEG:N }\end{array}$} & Protected vs. & \multicolumn{2}{|c|}{ Yield } \\
\cline { 2 - 4 } & Deprotected & Mono- & Bis- \\
\hline \multirow{2}{*}{$25: 75$} & PAOEt & $72 \%$ & $61 \%$ \\
\cline { 2 - 4 } & PAOH & $100 \%$ & $100 \%$ \\
\hline \multirow{2}{*}{$50: 50$} & PAOEt & $65 \%$ & $67 \%$ \\
\cline { 2 - 4 } & PAOH & $100 \%$ & $100 \%$ \\
\hline \multirow{2}{*}{$50: 45: 5\left(\mathrm{~N}_{3}\right)$} & $\mathrm{PAOEt}$ & & $76 \%$ \\
\cline { 2 - 4 } & $\mathrm{PAOH}$ & & $100 \%$ \\
\hline
\end{tabular}

Table S3 Summary of the Theoretical and Experimental Grafting Ratios for the Synthesis of PAPIMA-PEG based Polymers

\begin{tabular}{|c|c|c|c|}
\hline \multirow{2}{*}{$\begin{array}{c}\text { Type of Anchoring } \\
\text { Group }\end{array}$} & $\begin{array}{c}\text { Reagent Ratio } \\
\text { (PA:PEG) }\end{array}$ & \%PAOEt & Actual Grafting Ratios \\
\cline { 2 - 4 } & $25: 75$ & $23 \%$ & $77 \%$ \\
\hline MPAOEt-NH & $50: 50$ & $51 \%$ & $49 \%$ \\
\cline { 2 - 4 } & $25: 75$ & $19 \%$ & $81 \%$ \\
\hline \multirow{2}{*}{ BPAOEt-NH } & $50: 50$ & $54 \%$ & 46 \\
\cline { 2 - 4 } & \multicolumn{2}{|c}{} \\
\hline
\end{tabular}

\footnotetext{
${ }^{d}$ Note that we have only investigated the synthesis of bis-phosphonic acid-based azide polymers. The synthesis of mono-phosphonic acid-based azide polymers have been reported elsewhere ref [6].
} 


\subsection{Synthesis of AuNPs (13 nm) using the Turkevich-Frens Method from Ref [7]}

AuNPs in this investigation were prepared using the citrate reduction technique commonly described in literature. Briefly, a $250 \mathrm{~mL}$ Erlenmeyer flask equipped with a 1-inch stir bar and a glass rod was filled with $98 \mathrm{~mL}$ of Milli-Q filtered $\mathrm{H}_{2} \mathrm{O} .1 \mathrm{~mL}$ of trisodium citrate dihydrate $(3 \% \mathrm{w} / \mathrm{v})$ was added to this solution and the resulting mixture was brought to a boil under vigorous stirring. Next, a $1 \mathrm{~mL}$ aliquot of $\mathrm{HAuCl}_{4}(1 \% \mathrm{w} / \mathrm{v})$ was quickly added to the rapidly boiling solution. The reaction was allowed to proceed for approximately seven minutes before it was removed and cooled using an ice bath to $25^{\circ} \mathrm{C}$. At this point, the solution was noted to have a red-wine colour. The NP dispersion was then characterized using UV-vis, DLS, and TEM.

\subsection{General Ligand Exchange of AuNPs/AuNUs with MP- or BP-PIMA-PEG}

Excess citrate from synthesized gold nanoparticles was removed by using centrifugation concentration devices from Millipore (product code: UFC910024; MW cutoff of 100 kDa). Briefly, centrifugation devices were rinsed with $\mathrm{MQ}$ water for 15 mins at 5,000 x g ( $3 \mathrm{~mL}$ in a $15 \mathrm{~mL}$ device). The remaining MQ water was removed from the device and synthesized citrate stabilized gold nanoparticles were added to the filter. The nanoparticles were concentrated at 5,000 $\times \mathrm{g}$ for 15 minutes and excess citrate solution was removed. The concentrate gold nanoparticles were then diluted in autoclaved MQ water and used for ligand exchange.

Citrate stabilized AuNPs (13 nm) in Milli-Q $\mathrm{H}_{2} \mathrm{O}$ were diluted to $14 \mathrm{nM}$ and a final volume of $20 \mathrm{~mL}$ was in a 1 neck, $100 \mathrm{~mL}$ round bottom flask that contained a stir bar. Next, approximately $8 \mathrm{mg}$ of PAOH-PIMA-PEG polymer in $10 \mathrm{~mL}$ of $1 \times$ PBS buffer was added dropwise to the stirring solution of AuNPs. The reaction was then left to stir overnight at room temperature. Then the excess free ligands were removed via three rounds of centrifugation to 
the nanoparticle dispersion (diluting with Milli-Q $\mathrm{H}_{2} \mathrm{O}$ each time) over a centrifugal filtration device from Millipore (product code: UFC910024; MW cutoff of 100 kDa). ${ }^{e}$

\subsection{Generalized Ligand Exchange of QDs/IONPs with MP- or BP-PIMA-PEG}

Ligand exchange for QDs/IONPs was done by a biphasic ligand exchange strategy, with hydrophobic QDs/IONPs in the organic phase and polymer in the aqueous phase. Briefly, $125 \mu \mathrm{g}$ of quantum dots/IONPs in toluene (stock solution of $5 \mathrm{mg} / \mathrm{mL}$ ) were precipitated with $\sim 1 \mathrm{~mL}$ of $95 \%$ ethanol using centrifugation ( $12400 \times \mathrm{g}$ for 15 minutes in a $1.7 \mathrm{~mL}$ centrifuge tube). The resulting supernatant was discarded, and the pellet of quantum dots/IONPs was dissolved in $300 \mu \mathrm{L}$ of hexanes. Next, $125 \mu \mathrm{g}$ of polymer (mono- or bis- PAOH-PIMA-PEG 25:75 or 50:50) in $300 \mu \mathrm{L}$ of Milli-Q water was added dropwise to the solution of QDs followed by $15 \mu \mathrm{L}$ of tetramethyl ammonium hydroxide solution (25\% wt. solution in $\mathrm{MeOH}$ ). The mixture was then transferred to a glass vial and placed in an analog shaker overnight. The next day, the mixture was observed, and quantum dots/IONPs were noted to have transferred into the aqueous phase. The phases were separated, and the organic phase was disposed before further work-up. NPs coated with polymer were diluted with $\mathrm{MQ} \mathrm{H} \mathrm{H}_{2} \mathrm{O}$ before syringe filtration $(0.22 \mu \mathrm{m}$ sterile PES filters). NPs were concentrated from the solution of excess polymer via membrane centrifugation devices (product code: UFC510096; MW c/o of $100 \mathrm{kDa} 3770$ x g for 5 minutes). The resulting quantum dots/IONPs were diluted with $300 \mu \mathrm{L}$ of Milli-Q water and concentrated on membrane

\footnotetext{
e Technical Note: Gold nanoparticles without excess citrate are less stable and may aggregate during ligand exchange. To prevent aggregation, use glassware and stirbars that are washed with aqua regia and use the diluted gold nanoparticle solution shortly after preparation.
} 
centrifugation device a further two times (using the same centrifugation conditions as above). The QDs/IONPs were then characterized and used without further processing.

\subsection{Conjugation of QDs BP-PIMA-PEG-N 3 with DBCO-Cy3}

Preparation of QD-Dye conjugates was accomplished using QDs modified with BP-PIMAPEG-N $\mathrm{N}_{3}$ and DBCO-Cy3. Briefly, $50 \mu \mathrm{L}$ of $\sim 5 \mu \mathrm{M}$ of QDs was diluted in $1000 \mu \mathrm{L}$ of MQ $\mathrm{H}_{2} \mathrm{O}$. Separately, DBCO-Cy3 ( $1 \mathrm{mg}$ ) was dissolved in $1 \mathrm{~mL}$ of DMSO and the concentration was determined using UV-vis spectroscopy (2.5 mM). Next, 10 mol equivalents of DBCO-Cy3 solution was added to the QDs solution and the mixture was left mixing overnight. The next day, the QD conjugates were separated from excess DBCO dye using membrane centrifugation devices (product code: UFC510096; MW c/o of $100 \mathrm{kDa} ; 3770 \times \mathrm{g}$ for 5 minutes and 7 runs total) before further use.

\subsection{Colloidal Stability of AuNPs in Various Buffers}

Colloidal stability of AuNPs was investigated using UV-vis absorbance measurements and gel electrophoresis. AuNPs coated with M25, M50, B25, or B50 polymers were obtained after ligand exchange and their concentrations were determined using UV-vis spectroscopy. AuNP were diluted to a concentration of $8 \mathrm{nM}(300 \mu \mathrm{L})$ using stock solutions of concentrated buffers (e.g. $2 \mathrm{M} \mathrm{NaCl}, 10 \times \mathrm{PBS}$ ) and $\mathrm{MQ} \mathrm{H}_{2} \mathrm{O}$ to yield NP solutions in $1 \times \mathrm{PBS}, 5 \times \mathrm{PBS}, \mathrm{MQ} \mathrm{H}_{2} \mathrm{O}, 1 \mathrm{M} \mathrm{NaCl}$, and undiluted mouse serum. $50 \mu \mathrm{L}$ of the resulting NP solutions were transferred to a 384 well assay plate (black/clear bottom, non-treated, costar) for measurement with a plate reader (BioTek Cytation 3 microplate reader). 
The data from the plate reader was obtained as point measurements at $516 \mathrm{~nm}, 700 \mathrm{~nm}$, and spectral scans at $450-750 \mathrm{~nm}$ with $5 \mathrm{~nm}$ steps. ${ }^{f}$ The resulting intensity measurements $\left[I\left(A_{700 \mathrm{~nm}}\right), I\left(A_{516 \mathrm{~nm}}\right)\right]$ were used to obtain the aggregation factor (AF) for AuNPs, as shown in Equation S1. AF was plotted as a function of time to investigate the degree of aggregation due to the buffer environment (Figure 6 in main manuscript).

$$
\text { Equation S1 } \quad \text { Aggregation Factor }(A F)=\frac{I\left(A_{700 \mathrm{~nm}}\right)}{I\left(A_{516 \mathrm{~nm}}\right)}
$$

\subsection{Colloidal Stability of QDs in Various Buffers}

Colloidal stability of QDs was investigated using solution-based luminescence measurements and gel electrophoresis. QDs coated with M25, M50, B25, or B50 polymers were obtained after ligand exchange, and their concentrations were determined using UV-vis spectroscopy (refer to Table 1 for extinction coefficients for QDs). QDs were diluted to a concentration of $500 \mathrm{nM}(300 \mu \mathrm{L})$ using stock solutions of concentrated buffers (e.g. $2 \mathrm{M} \mathrm{NaCl}$, $10 \times \mathrm{PBS}$ ) and $\mathrm{MQ} \mathrm{H}_{2} \mathrm{O}$ to yield NP solutions in $1 \times \mathrm{PBS}, 5 \times \mathrm{PBS}, \mathrm{MQ} \mathrm{H}_{2} \mathrm{O}, 1 \mathrm{M} \mathrm{NaCl}$, and undiluted mouse serum. $50 \mu \mathrm{L}$ of the resulting NP solutions were transferred to a 384 well assay plate (black walls/bottom, non-treated, Grenier Bio-One) for measurement with a plate reader (BioTek Cytation 3 microplate reader). Additionally, $50 \mu \mathrm{L}$ of $0.02 \mathrm{mM}$ Fluorescein solution (pH 11) was used as an external standard to account for instrumental error via ratiometric analysis (Figure S1 shows the integrity of the external standard solution during colloidal stability investigations).

Equation S2

$$
\text { Ratiometric Intensity }(I)=\frac{\int_{650}^{450} I(Q D s)}{\left.\int_{650}^{450} I \text { (Fluorescein }\right)}
$$

\footnotetext{
${ }^{f}$ Note: All measurements were made with four replicates of each buffer condition.
} 
Equation S3 Normalized Intensity $(I)=\frac{\int_{650}^{450} I(Q D s)}{\int_{650}^{450} I_{o}(Q D s)} \times$ Ratiometric Intensity (I)

The data from the plate reader was obtained as point measurements at $490 \mathrm{~nm}, 520 \mathrm{~nm}$, $560 \mathrm{~nm}, 600 \mathrm{~nm}$, and spectral scans at $450-650 \mathrm{~nm}$ with $5 \mathrm{~nm}$ steps.g The luminescence measurements for the spectral scans were integrated over the wavelength range of interest and ratiometric analysis with external standard was used to obtain the instrument corrected signal for the buffer conditions (shown as Equation S2). Here, $\int_{650}^{450} I(Q D S)$ is the integrated intensity of QDs as a function of time and $\int_{650}^{450} I$ (Fluorescein) is the integrated intensity of the Fluorescein solution (0.02 mM at pH 11) was a function of time. Finally, the QD signal was normalized to the initial luminescence signal, using Equation S3, to track any loss of QD emission over time (Figure 7 in main manuscript). Here, $\int_{650}^{450} I(Q D s)$ is the integrated intensity of QDs as a function of time and $\int_{650}^{450} I_{o}(Q D s)$ is the integrated intensity of QDs at the first time measurement.

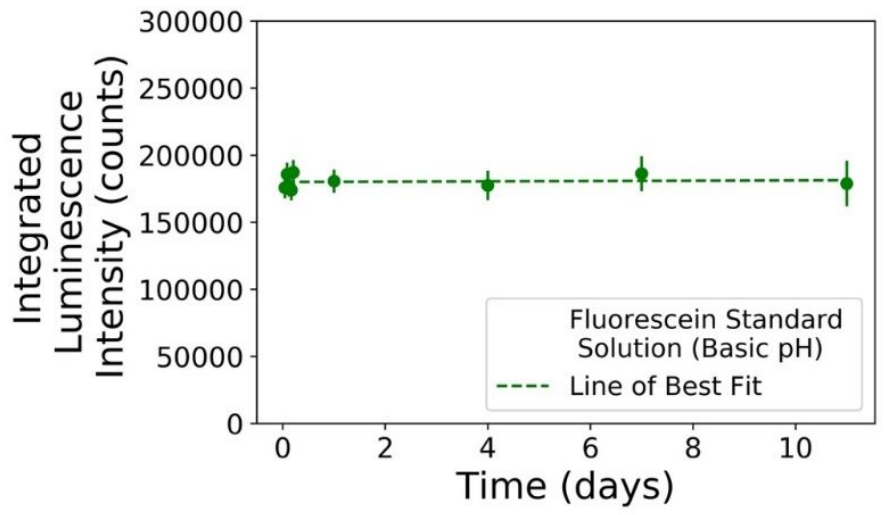

Figure S1 Integrated Luminescence Intensity (integrated signal over 450-650 nm) of Fluorescein Standard Solution ( $\mathrm{pH} \mathrm{11)} \mathrm{as} \mathrm{measured} \mathrm{by} \mathrm{the} \mathrm{BioTek} \mathrm{Cytation} 3$ microplate reader. Line of best

g Note: All measurements were made with four replicates of each buffer condition. 
fit: $y=(112) x+180000$. Signal integrity was retained over the span of the investigations of the colloidal stabilities.

\section{Characterization of AuNPs synthesized using the Turkevich-Frens Method}

The TEM, normalized UV-vis absorption spectra, and DLS profile of synthesized gold nanoparticles, are presented in Figures S2A-E respectively. From these different techniques, the size and shape of gold nanoparticles was confirmed to be $13 \mathrm{~nm} \pm 2 \mathrm{~nm}$ and spherical.
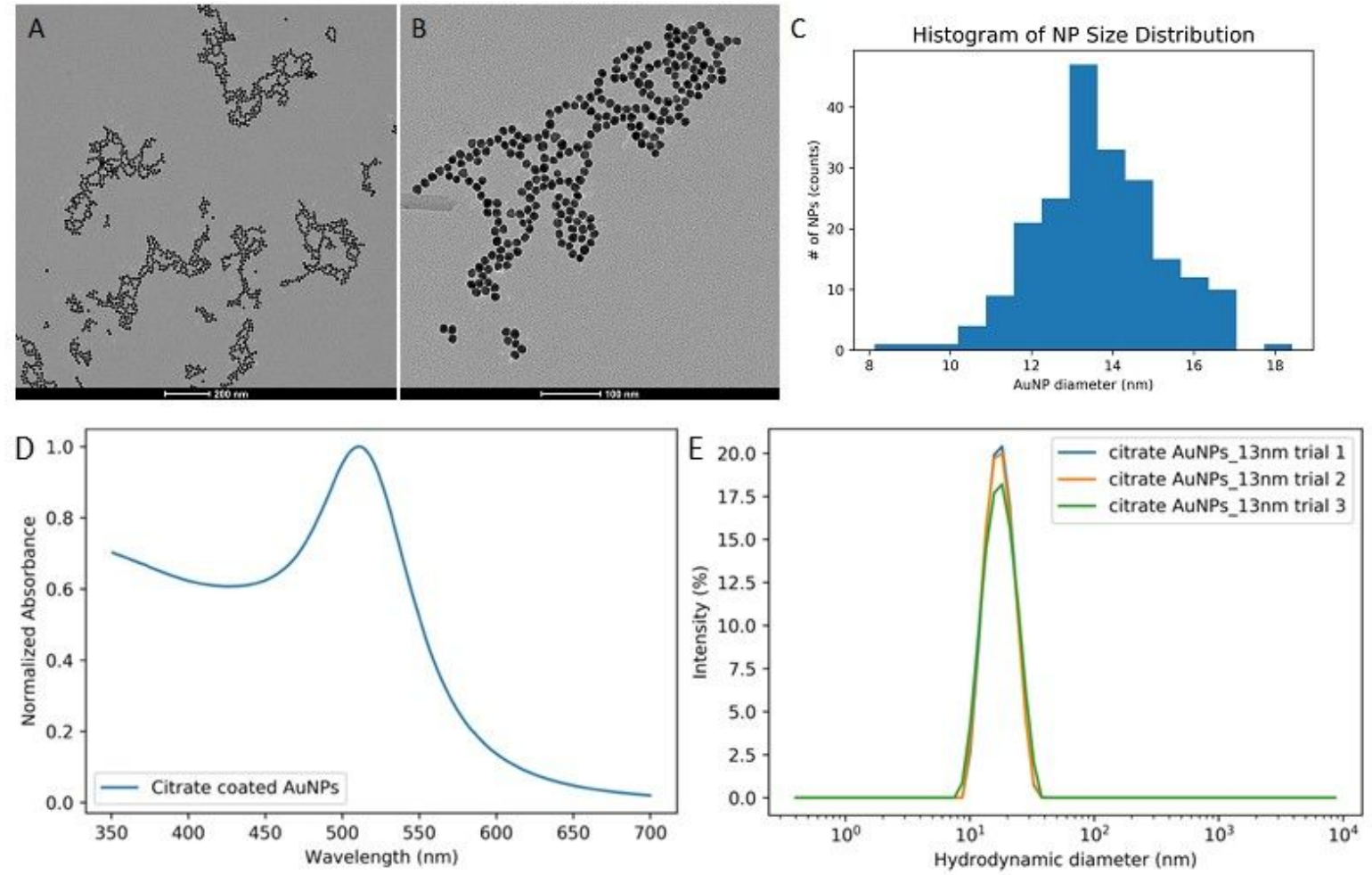

Figure S2 Transmission electron microscopy (TEM) images of citrate coated AuNPs in $M Q \mathrm{H}_{2} \mathrm{O}$. A. wide field image of the synthesized AuNPs (scale bar was $200 \mathrm{~nm}$ ). B. zoomed in image of the synthesized AuNPs (scale bar was $100 \mathrm{~nm}$ ). C. Histogram of synthesized nanoparticle diameters (average diameter was $13 \mathrm{~nm}$, standard deviation of $2 \mathrm{~nm}$ ). D. Normalized UV-vis spectra of citrate coated gold nanoparticles (AuNPs). Here, the LSPR peak was at $511 \mathrm{~nm}$. E. Dynamic light scattering data of citrate coated gold nanoparticles (AuNPs). Note that three trials were conducted for the gold nanoparticles for an average hydrodynamic diameter $\left(d_{H}\right)$ of $16.6 \mathrm{~nm} \pm$ 0.1 and an average PDI of $0.06 \pm 0.01$. 


\section{Characterization of Citrate Stabilized AuNUs $(50,60,80,100 \mathrm{~nm})$}

AuNUs coated with citrate were characterized using UV-vis spectroscopy, DLS, and TEM, data is presented as Figures S3A-F. From Figure S3, the hydrodynamic diameter (HD) and PDI of the various AuNUs were found to be: AuNU_50 nm_HD was $59 \pm 1$ (PDI = $0.12 \pm 0.005)$, AuNU_60 nm_HD was $68 \pm 2(P D I=0.12 \pm 0.005)$, AuNU_80 nm_HD was $92 \pm 3$ (PDI = 0.1 \pm 0.02), AuNU_100 nm_HD was $114 \pm 3($ PDI $=0.07 \pm 0.02)$
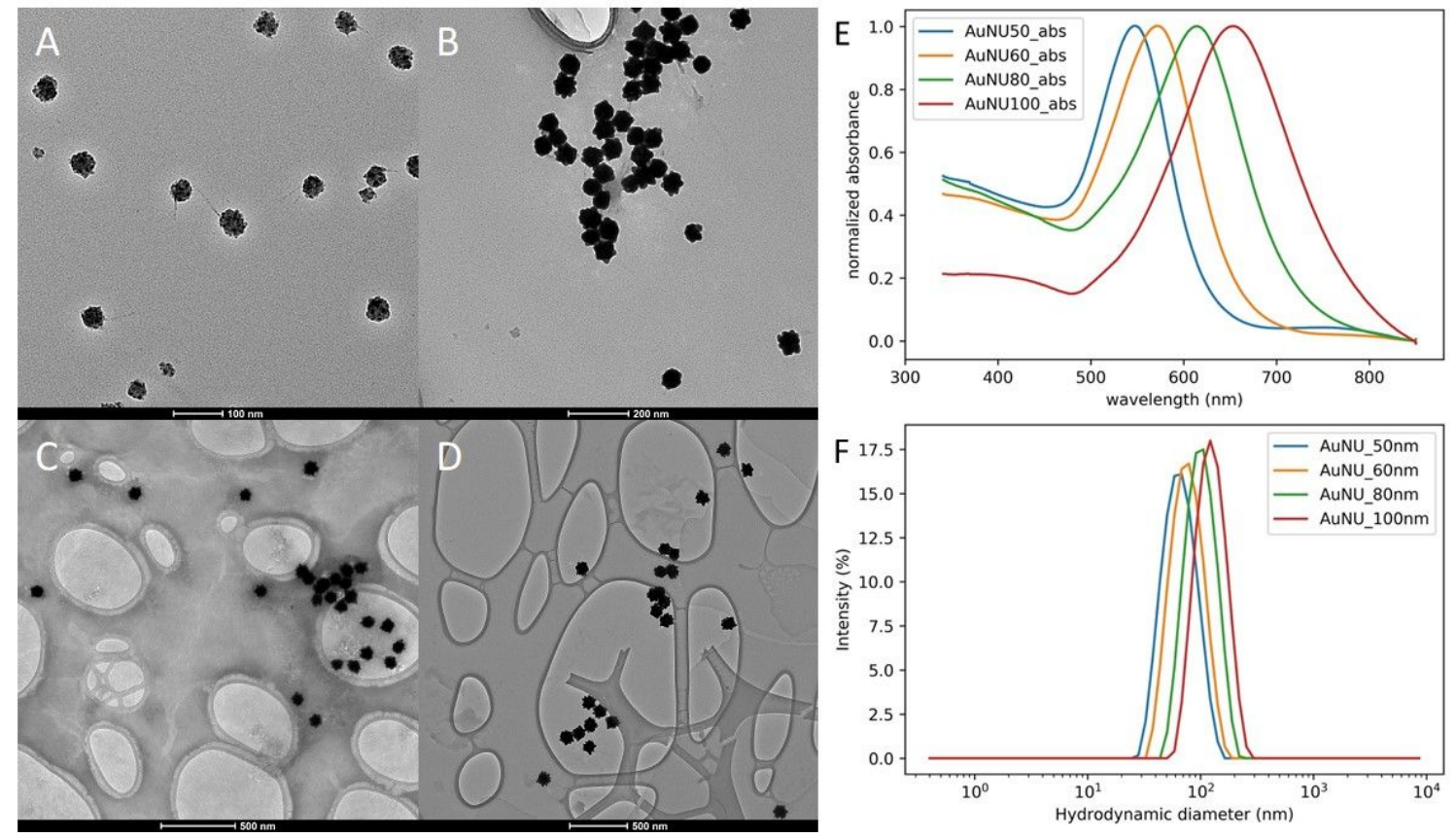

Figure S3 Transmission electron microscopy images of citrate stabilized gold nanourchins on carbon grids A. $50 \mathrm{~nm}$, B. $60 \mathrm{~nm}$, C. $80 \mathrm{~nm}$, D. $100 \mathrm{~nm}$. The scalebars were $100 \mathrm{~nm}$ (AuNU_50), 200 nm (AuNU_60), and 500 nm (for AuNU_80, AuNU_100), respectively. E. Normalized UV-vis spectra of citrate stabilized gold nanourchins. The LSPR peaks were found to be at $545,570,612$, and $650 \mathrm{~nm}$ for AuNUs $50 \mathrm{~nm}, 60 \mathrm{~nm}, 80 \mathrm{~nm}$, and $100 \mathrm{~nm}$, respectively. F. Dynamic light scattering data of citrate coated gold nanourchins. Note that one of the three trials are shown for each of the different sized AuNUs. The average hydrodynamic diameter ( $\mathrm{nm}$ ) and PDI values are shown in Table 3 of the main manuscript. 


\section{Characterization of Polymer-coated AuNUs (50, 60, 80, $100 \mathrm{~nm}$ )}

AuNUs coated with BPAOH-PIMA-PEG polymer were characterized using UV-vis spectroscopy, DLS, and TEM. The data are presented as Figures S4A-G. From Figure S4, the hydrodynamic diameter (HD) and PDI of the various AuNUs were found to be: AuNU_50 nm_HD was $76 \pm 2(P D I=0.206 \pm 0.07)$, AuNU_60 nm_HD was $87 \pm 3($ PDI $=0.135 \pm 0.015)$ AuNU_80 nm_HD was $99 \pm 3(P D I=0.09 \pm 0.01), A u N U \_100$ nm_HD was $124 \pm 3.5$ $(P D I=0.07 \pm 0.02)$
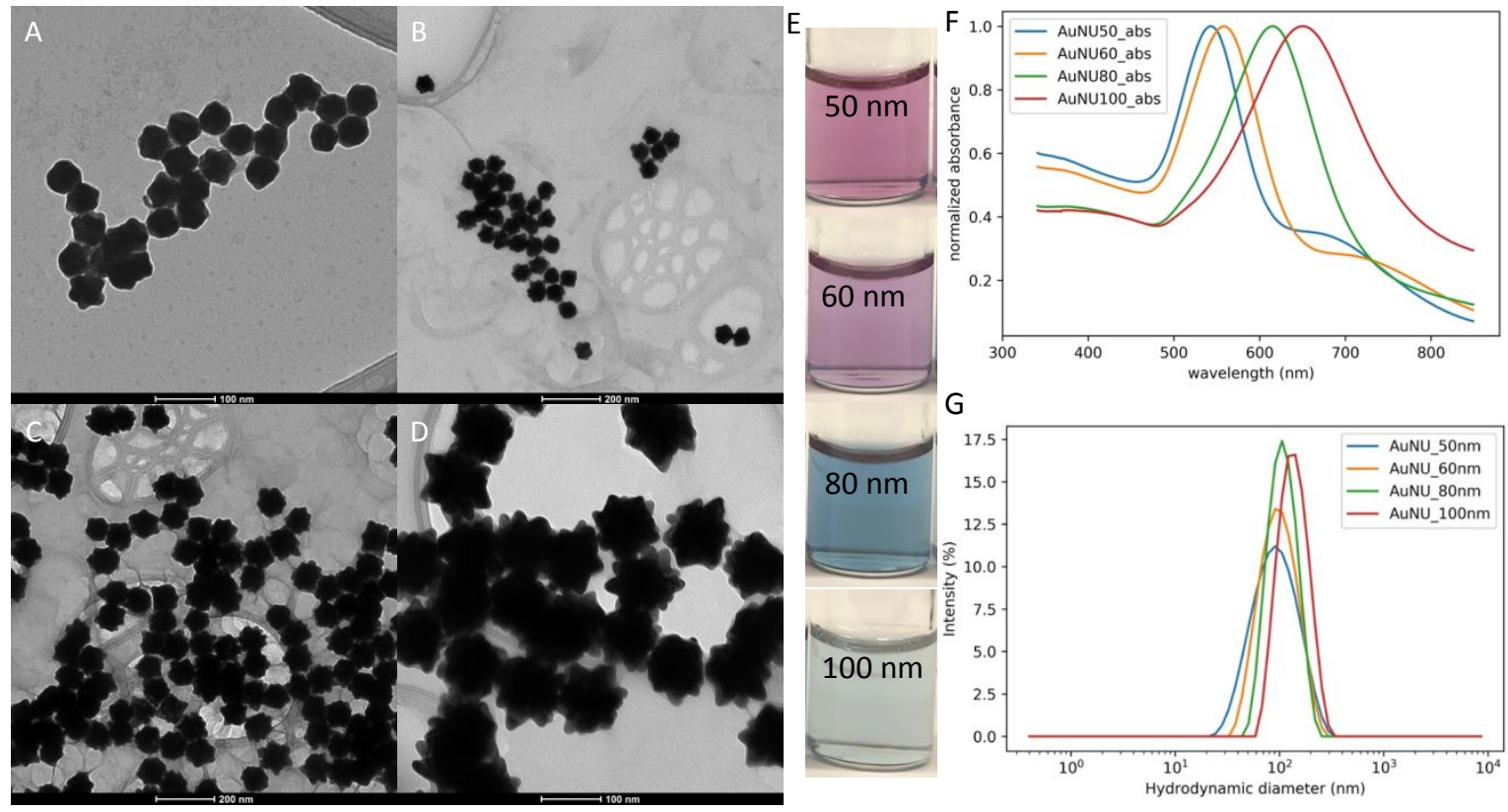

Figure S4 presents transmission electron microscopy images of BP-PIMA-PEG stabilized gold nanourchins (AuNUs) on carbon lacey grids A. $50 \mathrm{~nm}$, B. $60 \mathrm{~nm}$, C. $80 \mathrm{~nm}$, D. $100 \mathrm{~nm}$. The scalebars were $100 \mathrm{~nm}$ (AuNU_50), $200 \mathrm{~nm}$ (AuNU_60), $200 \mathrm{~nm}$ (AuNU_80), and $100 \mathrm{~nm}$ (AuNU_100), respectively. E. Camera images of BPA-PIMA-PEG stabilized gold nanourchins in MQ $\mathrm{H}_{2} \mathrm{O}$ (from top to bottom) $50 \mathrm{~nm}, 60 \mathrm{~nm}, 80 \mathrm{~nm}, 100 \mathrm{~nm}$. The images were taken using an iPhone 8 (Apple, California, U.S.A.). F. Normalized UV-vis spectra of citrate stabilized gold nanourchins. The LSPR peaks were found to be at 545, 570, 612, and $650 \mathrm{~nm}$ for AuNU $50 \mathrm{~nm}$, $60 \mathrm{~nm}, 80 \mathrm{~nm}$, and $100 \mathrm{~nm}$, respectively. G. Dynamic light scattering data of citrate coated gold 
nanourchins. Note that one of the three trials were shown in this figure for each of the different sized AuNUs.

7. Characterization of Iron Oxide Nanoparticles coated with Oleic Acid and BPPIMA-PEG (IONPS)

IONPs were characterized in the organic and also in aqueous/polymer material using DLS and TEM. From Figure S5A-C, the hydrodynamic diameter (HD) and PDI of the IONPs were found to be: IONP_oleic-acid_HD was $26.4 \pm 0.9$ (PDI $=0.34 \pm 0.03)$, and IONP_BPAOH-PIMAPEG_HD was $20.1 \pm 0.5(\mathrm{PDI}=0.27 \pm 0.01)$
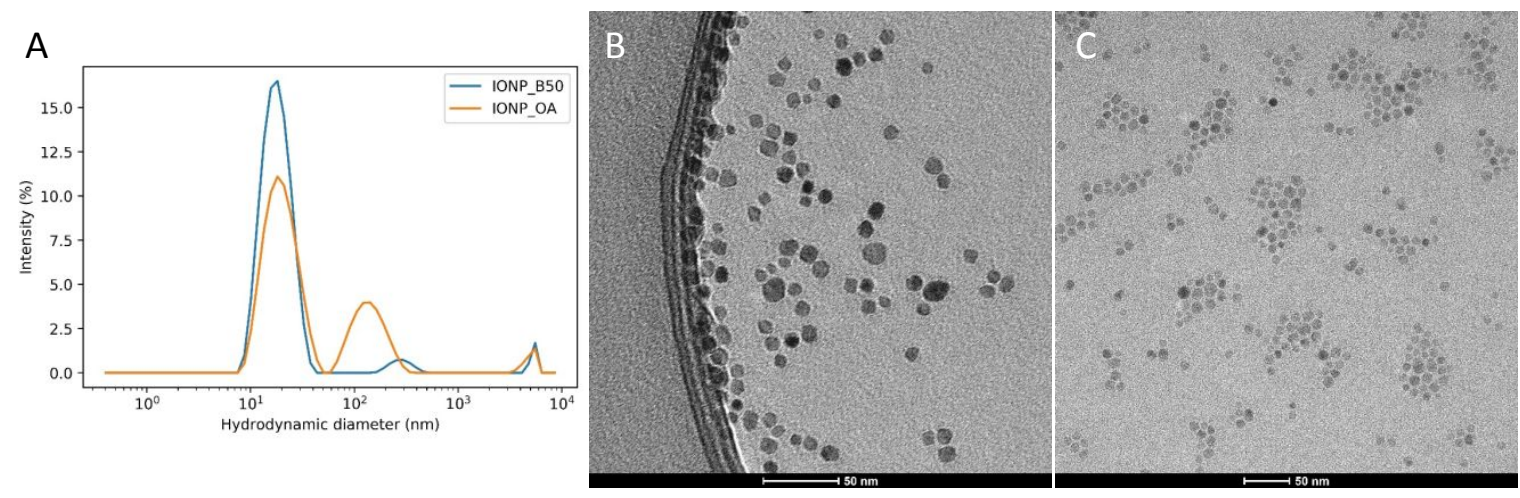

Figure S5A shows DLS data for IONPs coated with oleic acid and B50 polymer. B. Transmission electron microscopy images of iron oxide nanoparticles coated with oleic acid on carbon grids (scale bar is $50 \mathrm{~nm}$ ). C. Transmission electron microscopy images of iron oxide nanoparticles coated with BPAOH-PIMA-PEG (B50) on carbon grids (scale bar is $50 \mathrm{~nm}$ ). 


\section{Characterization of Alloyed Quantum Dots coated with Oleic Acid and with}

\section{Various Polymers (CdSeS/ZnS)}

The absorption and luminescence profiles of three different types of alloyed quantum dots was presented in Figure 5 of the main manuscript. Note that the alloyed quantum dots were initially coated with oleic acid. The TEM image for these quantum dots is also shown here in Figure S6. Quantum dots stabilized with various phosphonic acid-based polymers were characterized by UV-visible spectroscopy, luminescence spectroscopy, and gel electrophoresis. Spectroscopic analysis of QDs after ligand exchange produced absorption and luminescence profiles with shifted peaks relating to the first exciton, and for the wavelength for maximum emissions. These spectral shifts corresponded to the changes of surface chemistry for the nanocrystals (as related to the exchange of oleic acids with PIMA polymers) and are shown in Figures $5 \mathrm{~F}-\mathrm{G}$ and S7. A summary of the luminescence properties of polymer coated QDs is presented in Table S4.

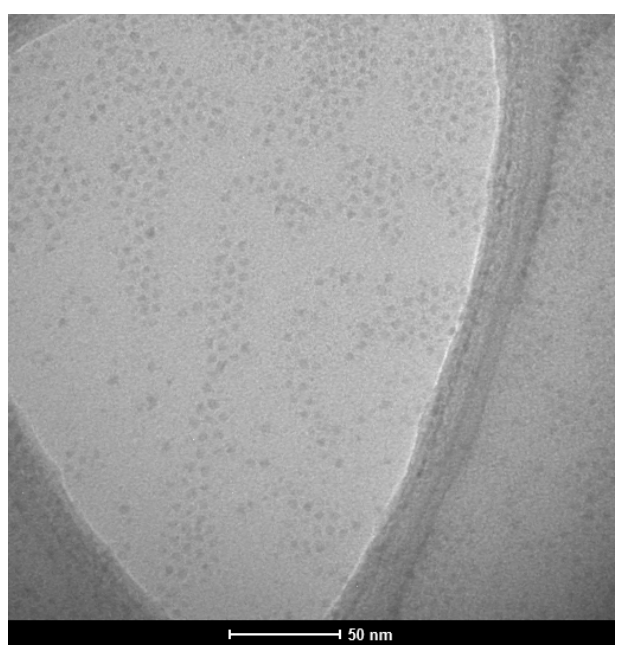

Figure $\mathbf{S 3}$ presents transmission electron microscopy images of oleic acid stabilized quantum dots on carbon grids (scale bar is $50 \mathrm{~nm}$ ). Here, the QDs were determined to be $5.3 \pm 0.6 \mathrm{~nm}$ in diameter from an average of 51 measurements. 

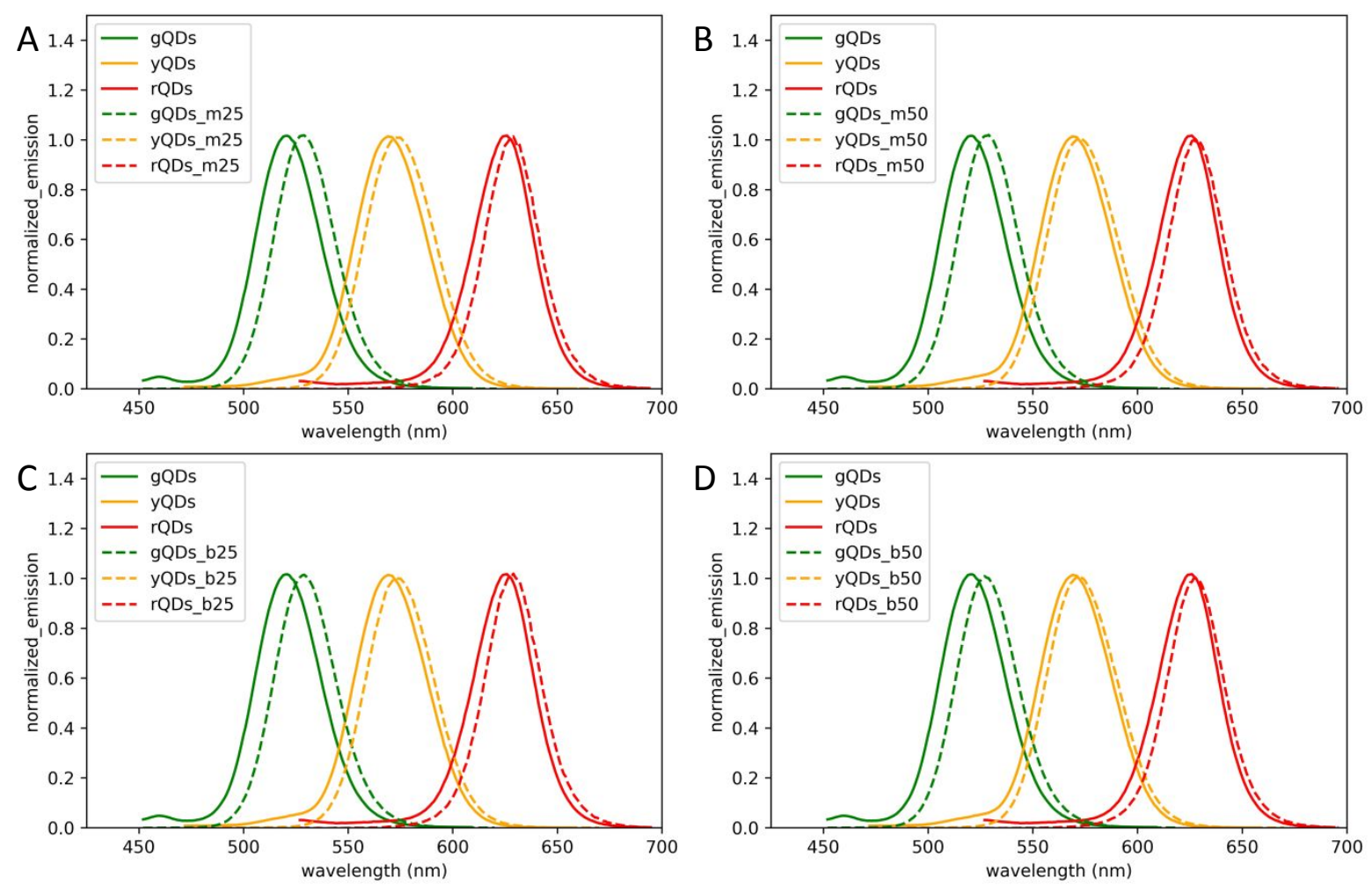

Figure S7 shows luminescence spectra for green, yellow, and red quantum dots (gQDs, yQDs, RQDs respectively) modified with mono- or bis-PAOH-PIMA-PEG normalized to the peak luminescence intensity. The solid lines represent the luminescence profile of oleic acid capped QDs and the dashed lines represent the QDs coated with PIMA polymer. A. M25, B. M50, C. B25, and D. B50.

Table S4 Peak Emission for Alloyed Semiconductor Nanocrystals CdSeS/ZnS Post Ligand Exchange

\begin{tabular}{|l|c|l|c|c|c|}
\hline $\begin{array}{l}\text { Quantum } \\
\text { Dots }\end{array}$ & $\begin{array}{l}\text { Organic QDs } \\
\text { (OA coating) } \\
\mathrm{nm}\end{array}$ & $\begin{array}{l}\text { Mono-PAOH- } \\
\text { PIMA-PEG } \\
(25: 75) \mathrm{nm}\end{array}$ & $\begin{array}{l}\text { Mono-PAOH- } \\
\text { PIMA-PEG } \\
(50: 50) \mathrm{nm}\end{array}$ & $\begin{array}{l}\text { Bis-PAOH- } \\
\text { PIMA-PEG } \\
(25: 75) \mathrm{nm}\end{array}$ & $\begin{array}{l}\text { Bis-PAOH- } \\
\text { PIMA-PEG } \\
(50: 50) \mathrm{nm}\end{array}$ \\
\hline QD525 & 521 & 530 & 530 & 530 & 528 \\
\hline QD570 & 569 & 575 & 573 & 564 & 573 \\
\hline QD630 & 626 & 629 & 628 & 630 & 628 \\
\hline
\end{tabular}


Confirmation of ligand exchange was also done using gel electrophoresis, where polymer coated QDs were loaded onto $1 \%$ Agarose gel slabs using 1× Tris Borate EDTA buffer (TBE, 44.5 $\mathrm{mM}$ tris borate, $1 \mathrm{mM}$ EDTA) as the running buffer. QD samples diluted to a concentration of $100 \mathrm{nM}$ before being diluted to a final concentration of $40 \mathrm{nM}$ with $\mathrm{MQ}$ water and $40 \%$ sucrose. The gel experiments were run for 30 minutes (maximum) at an electric field strength of $2.5 \mathrm{~V}$ $\mathrm{cm}^{-1}$. The resulting images were captured by use of a BioRad Molecular Imager ${ }^{\circledR}$ GeIDoc $^{\top M}$ XR and processed by ImageJ (version 1.49u, National Institute of Health, USA). Results of the gel electrophoresis experiments are presented as Figure 5C-E.

\section{Colloidal Stability of AuNPs and QDs in Biologically Relevant Buffers}

In addition to the spectroscopic investigations of AuNPs and QDs in various buffers, colloidal stability was studied using gel electrophoresis and transmission electron microscopy. Below are Figures S8-S11 which show the stability of AuNPs and QDs coated with M25, M50, B25, and $\mathrm{B} 50$ polymers in $\mathrm{MQ} \mathrm{H}_{2} \mathrm{O}, 1 \mathrm{M} \mathrm{NaCl}, 1 \times$ PBS, $5 \times$ PBS buffers, and Mouse serum.

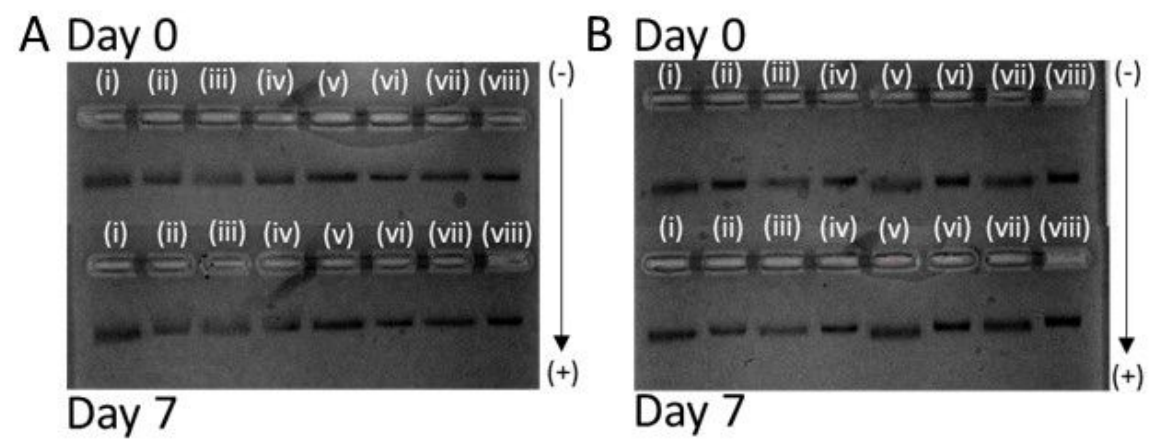

Figure S8A shows gel electrophoresis of AuNPs coated with (i, ii, iii, iv) M25, and (v, vi, vii, viii) M50 polymers. Samples were incubated in (i, v) $\mathrm{MQ} \mathrm{H}_{2} \mathrm{O}$, (ii, vi) $1 \mathrm{M} \mathrm{NaCl}$, (iii, vii) $1 \times$ PBS, and (iv, viii) $5 \times$ PBS buffers. Colloidal stability was measured on day 0 (fresh) and day 7. Electrode polarity is indicated on the right. B. presents gel electrophoresis of AuNPs coated with (i, ii, iii, iv) M25, and (v, vi, vii, viii) M50 polymers. Samples were incubated in (i, v) MQ $\mathrm{H}_{2} \mathrm{O}$, (ii, vi) $1 \mathrm{M} \mathrm{NaCl}$, (iii, 
vii) 1x PBS, and (iv, viii) 5x PBS buffers. Colloidal stability was measured on day 0 (fresh) and day

7. Electrode polarity is indicated on the right.

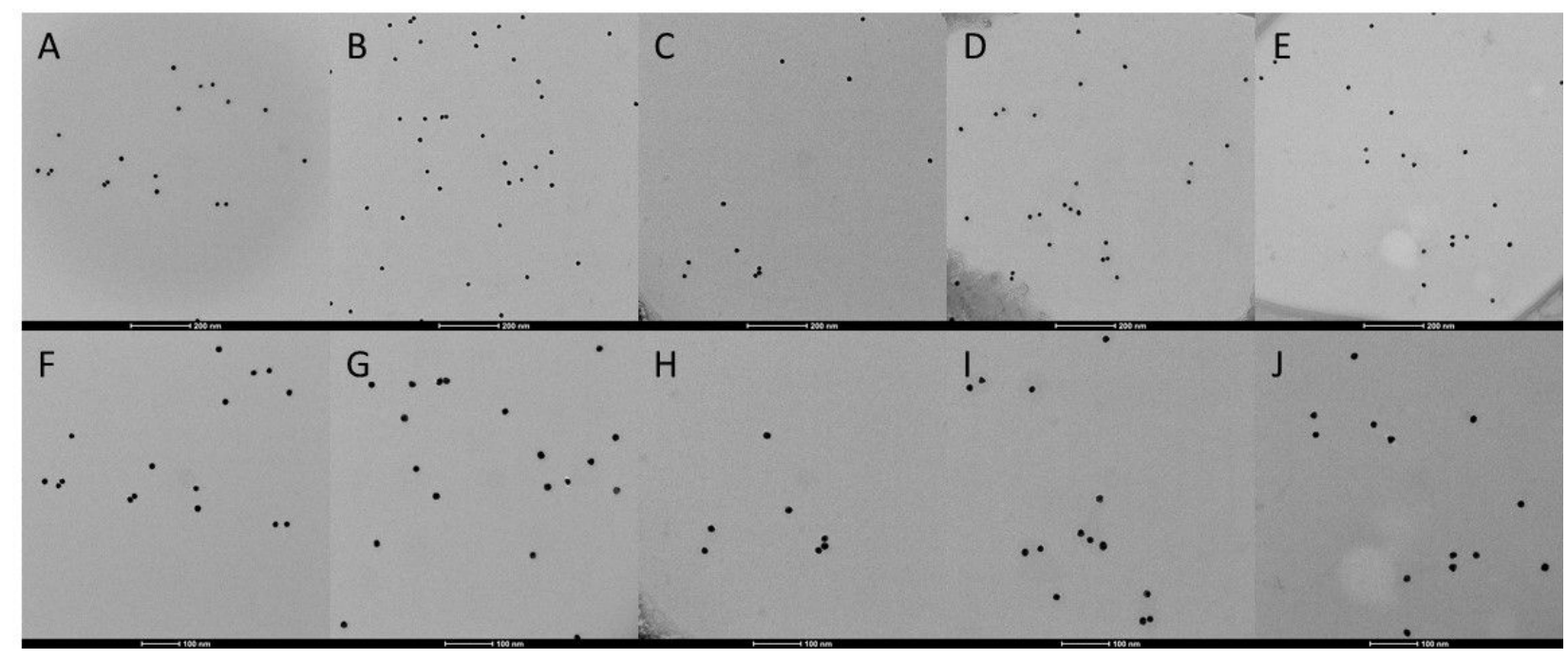

Figure S9A-E shows wide-field TEM images of AuNPs coated with B50 polymer, scalebar $200 \mathrm{~nm}$.

F-J are the same NPs, but the images are zoomed in for greater clarity, scalebar is $100 \mathrm{~nm}$. Here, NPs were incubated for 48 hours in the following buffers: A, F. MQ $\mathrm{H}_{2} \mathrm{O}, \mathbf{B}, \mathbf{G} .1 \mathrm{M} \mathrm{NaCl}, \mathbf{C}, \mathbf{H} .1 \times$ PBS, D, I. 5× PBS, and E, J. Mouse Serum.

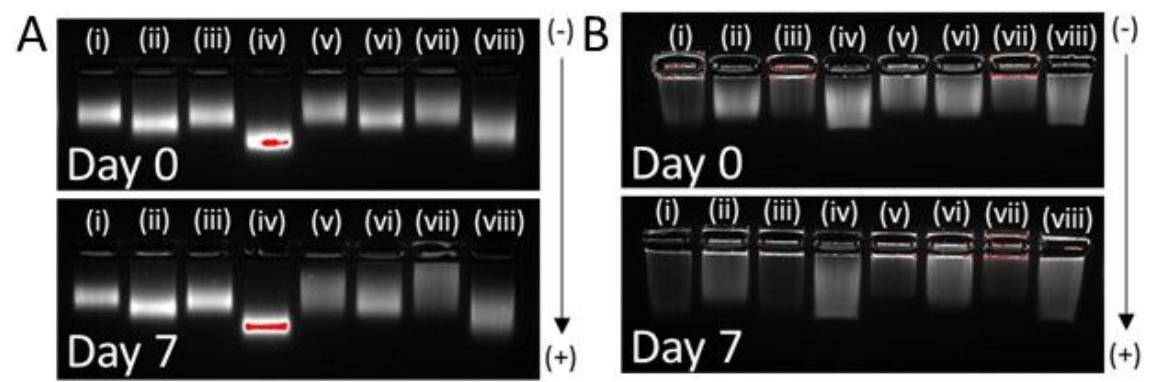

Figure S10A. shows gel electrophoresis of QDs coated with (i, v) M25, (ii, vi) M50, (iii, vii) B25, and (iv, viii) B50 polymers. Samples were incubated in (i, ii, iii, iv) $M Q \mathrm{H}_{2} \mathrm{O}$ and (v, vi, vii, viii) $1 \times$ PBS buffer. Colloidal stability was measured on day 0 (fresh) and day 7. Electrode polarity is indicated on the right. B. presents gel electrophoresis of QDs coated with (i, v) M25, (ii, vi) M50, (iii, vii) B25, and (iv, viii) B50 polymers. Samples were incubated in (i, ii, iii, iv) 5× PBS and (v, vi, 
vii, viii) $1 \mathrm{M} \mathrm{NaCl}$ buffer. Colloidal stability was measured on day 0 (fresh) and day 7 . Electrode polarity is indicated on the right.

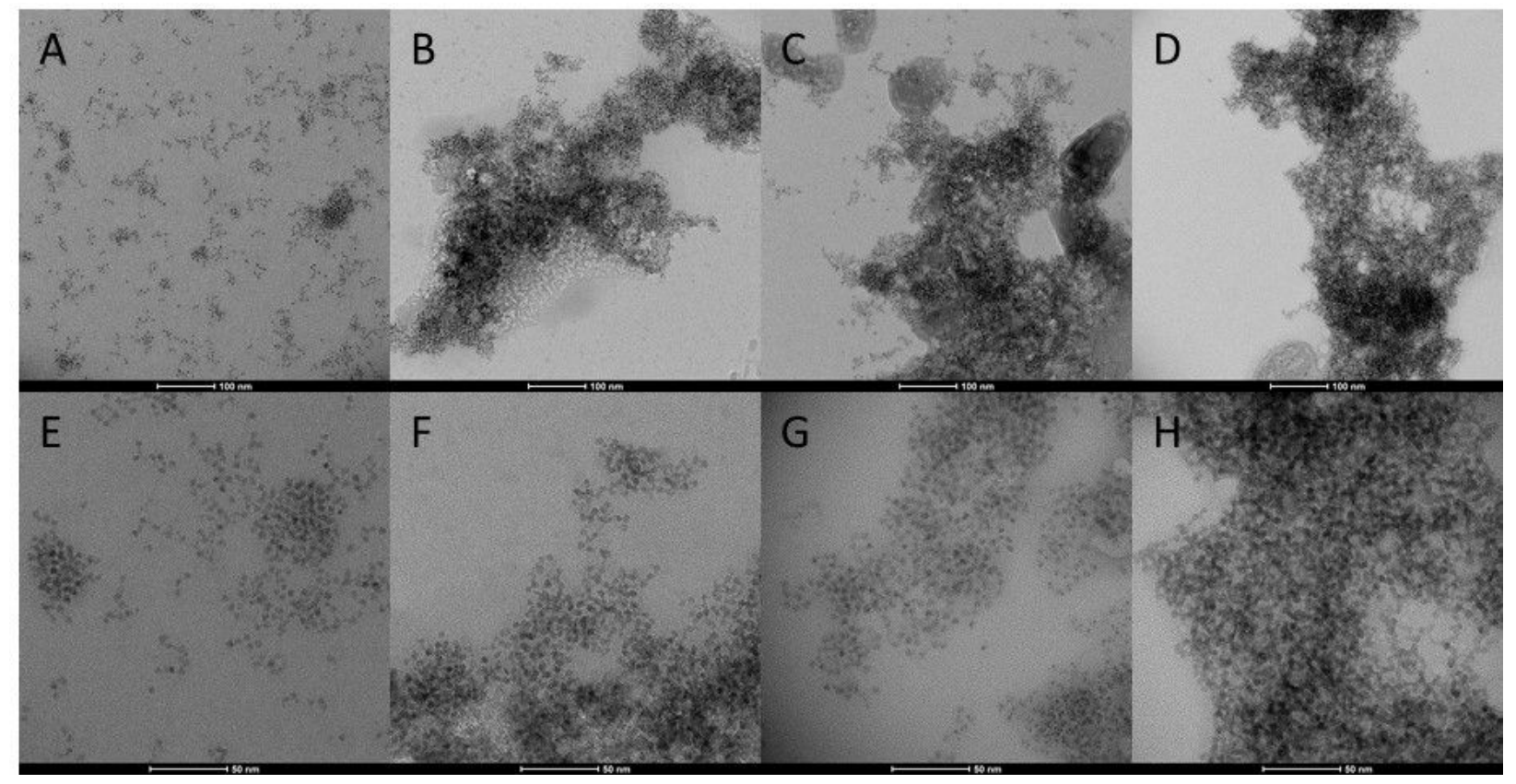

Figure S11A-D shows wide-field TEM images of AuNPs coated with B50 polymer, scalebar 100 $\mathrm{nm}$. E-H are the same NPs, but the images are zoomed in for greater clarity, scalebar is $50 \mathrm{~nm}$. Here, NPs were incubated for 48 hours in the following buffers: A, E. MQ $\mathrm{H}_{2} \mathrm{O}$, B, F. $1 \mathrm{M} \mathrm{NaCl}$, C, G. $1 \times$ PBS, and D, H. $5 \times$ PBS.

From Figure S9, we can see good stability for AuNPs coated with B50 polymer after they have been incubated in buffer for 48 hours. These results agree with the gel electrophoresis data from Figure $S 8$ and with the UV-vis spectroscopy data shown in Figure 6 of the main manuscript. Figure S11 shows the stability of QDs coated with B50 polymer after they have been incubated in the various buffers for 48 hours. Here, the QDs in $\mathrm{MQ} \mathrm{H}_{2} \mathrm{O}$ have relatively good stability as compared to QDs in the other buffers like $1 \mathrm{M} \mathrm{NaCl}, 1 \times \mathrm{PBS}$, and $5 \times \mathrm{PBS}$. These results are supported by the gel images in Figure S10 and the luminescence studies in Figure 7. 


\section{Quantum Yield Determination}

The quantum yield $(\Phi)$ of green emitting QDs stabilized with B50 polymer was determined using the single point approximation method [8] as shown in Equation S4. The $\Phi$ value represented quantum yield, $\mathrm{OD}(\lambda)$ represented absorption (in counts) at a specific wavelength ( $\lambda$ ), I represented the integrated fluorescence intensity (counts; as determined by trapezoidal approximation), $\mathrm{n}$ represented the refractive index, and the subscript $\mathrm{R}$ represented the reference fluorophore (with the known $\Phi$ ).

The reference fluorophore chosen for this determination was fluorescein (in $0.1 \mathrm{M} \mathrm{NaOH}$ ) at $\mathrm{pH} 11$ which has a reference $\Phi$ of 0.925 . The absorption wavelengths used for this determination were $406 \mathrm{~nm}$ and $470 \mathrm{~nm}$ for gQDs and fluorescein respectively. These same wavelengths were used for excitation of the gQDs and fluorescein when determining their integrated fluorescence intensities. The concentrations of gQDs and fluorescein used in all measurements were $250 \mathrm{nM}$ and $5 \mu \mathrm{M}$, respectively and the experimental $\Phi$ for $\mathrm{BN}_{3}$ coated QDs was found to be $45 \%$.

Equation S4

$$
\Phi=\Phi_{R}\left(\frac{I}{I_{R}}\right)\left(\frac{O D_{R}}{O D}\right)\left(\frac{n^{2}}{n_{R}^{2}}\right)
$$

\section{FRET Formalism}

Green emitting QDs stabilized with $\mathrm{BN}_{3}$ polymers were functionalized with Cy3-DBCO dye molecules using the azide groups on the polymer (copper-free click chemistry). Conjugation of dye molecules to QDs was confirmed using lifetime analysis of the QD-Cy3 conjugates. The Förster formalism was performed on these QD-Cy3 conjugates and values like the spectral overlap interval $(J)$ and Förster distance were determined using Equations S5 and S6. In Equation 
S5, $F_{D}$ was the fluorescence intensity associated with the donor, $\varepsilon_{A}$ was the molar extinction coefficient associated with the FRET acceptor, and $\lambda$ was the wavelength. Using Equation $\mathrm{S} 5$, the spectral overlap integral was calculated to be $8.1 \times 10^{15} \mathrm{M}^{-1} \mathrm{~cm}^{-1} \mathrm{~nm}^{4}$, as represented by the shaded region in Figure S12.

The Förster distance $\left(R_{0}\right)$ for the QD-Cy3 FRET pair was determined using the spectral overlap integral and Equation S6. Here, $n$ was refractive index of the surrounding medium (a value of 1.33 was used), $\kappa^{2}$ was the orientation factor (a random orientation was assumed, and a value of 2/3 was assigned) and the quantum yield $\left(\Phi_{D}\right)$ of $\mathrm{BN}_{3}$ QDs was earlier determined to be $45 \%$. Using this equation, the Förster distance was determined to be $6.4 \mathrm{~nm}$.

Equation S5

$$
J=\frac{\int F_{D}(\lambda) \varepsilon_{A}(\lambda) \lambda^{4} d \lambda}{\int F_{D}(\lambda) d \lambda}
$$

Equation S6

$$
R_{o}=0.211\left(\kappa^{2} n^{-4} \Phi_{D} J(\lambda)\right)^{1 / 6}
$$

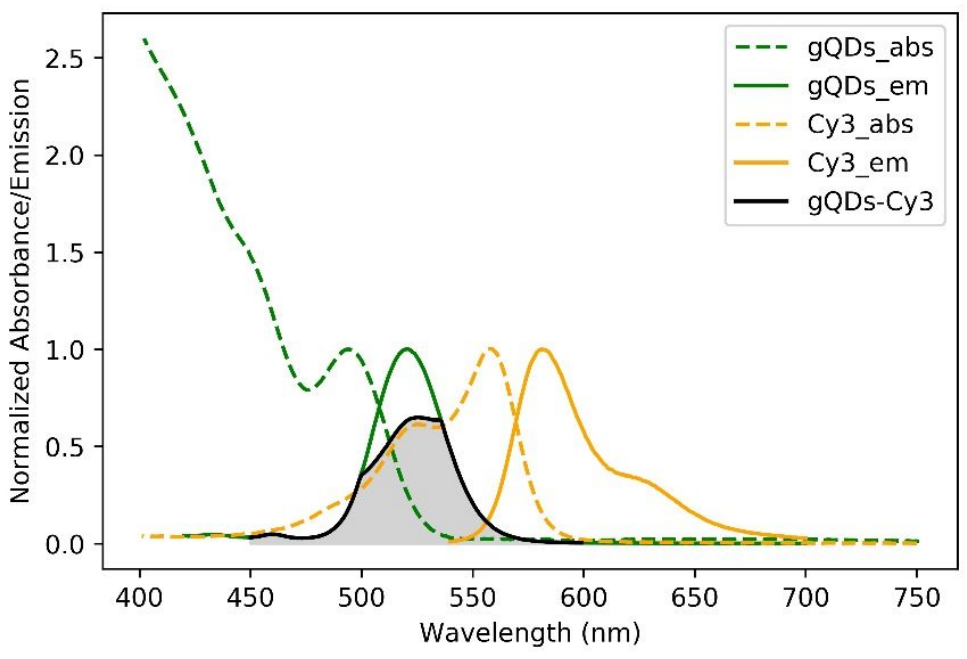

Figure S12 UV-vis and fluorescence data for $\mathrm{BN}_{3}$ coated quantum dots (CdSeS/ZnS) and DBCOCy3. The dashed lines represent the absorption spectra and the solid lines represent the emission profile. The shaded region represents the spectral overlap of the quantum dots and Cy3 dye. 


\section{Determination of Luminescence Decay and FRET Efficiency for gQD-Cy3 Pair}

The FRET efficiency $\left(E_{F R E T}\right)$ of gQDs-Cy3 conjugates were determined by measuring changes in the lifetime of the QD emission in the absence and presence of Cy3 dye molecules. Lifetime for these gQD or GQD-Cy3 conjugates were determined using the stroboscopic method with an excitation wavelength of $402 \mathrm{~nm}$ and an emission wavelength of $530 \mathrm{~nm}$. The resulting normalized lifetimes are presented in Figure S13 with their associated instrument response function (IRF). The $E_{F R E T}$ was calculated for the FRET pair using Equation S7; where $\tau_{D A}$ was the lifetime of the donor when acceptors were present, and $\tau_{D}$ was the lifetime of the donor when acceptors were absent.

Equation S5

$$
E_{F R E T}=1-\frac{\tau_{D A}}{\tau_{D}}
$$

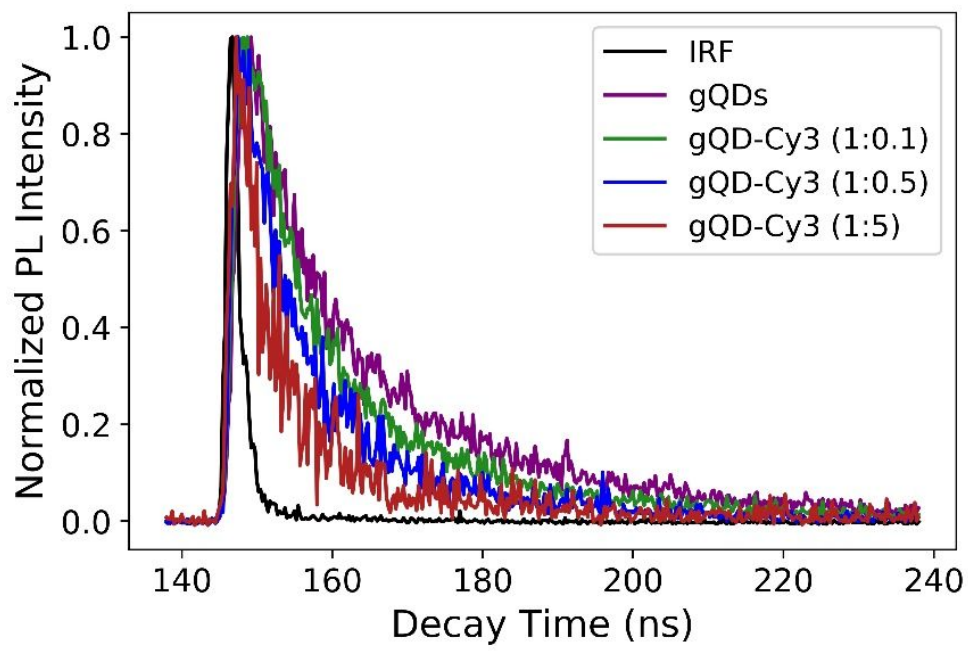

Figure S13 shows normalized PL decay profiles used for calculating the FRET efficiency of QDs conjugated with $\mathrm{Cy} 3$ dye molecules. The black trace represents the instrument response function (IRF), the purple trace represents the $\mathrm{BN}_{3}$ coated QDs, the green trace represents gQDs-Cy3 at a conjugation ratio of 1:0.1, the blue trace represents gQDs-Cy3 at a conjugation ratio of 1:0.5, and the red trace represents gQDs-Cy3 at a conjugation ratio of 1:5. 
In order to determine the dye loading capacity of the gQDs- $\mathrm{BN}_{3}$, different stoichiometric amounts of DBCO-Cy3 were incubated with the NPs. Specifically, DBCO-Cy3 was added at 0.1x, $0.5 \times$, and $5 \times$ molar quantities to gQDs. NPs were purified from free dye molecules using centrifugal filtration (as described in page S14) and analyzed for lifetime. Data analysis for the gQDs-Cy3 was accomplished using the Felix GX software by PTI and a mono-exponential and biexponential decay fit was performed. The resulting summary of decay times for the different samples of gQDs is presented in Table S5. Here, the FRET efficiencies were calculated using the mono-exponential decay time and the weighted average decay times yielded FRET efficiencies from $20-53 \%$ for the mono-exponential fit and $28-47 \%$ for the bi-exponential fit. In both cases, the average $E_{F R E T}$ for the 1:0.1, 1:0.5, and 1:5 gQDs-Cy3 sample were found to be approximately $20-28 \%, 33-42 \%$, and $47-53 \%$, respectively.

Table S5 Determination of Luminescence Decay Times and Associated FRET efficiencies for QDs coated with BPAOH-PIMA-PEG-N $\mathrm{N}_{3}$ and Conjugated with DBCO-Cy3.

\begin{tabular}{|l|l|l|l|l|l|l|}
\hline Sample $^{\mathrm{a}}$ & Mono. $\tau^{\mathrm{b}}$ & FRET Eff. $^{\mathrm{c}}$ & ${\text { Short } \tau(\%)^{\mathrm{d}}}^{\mathrm{L}}$ & ${\text { Long } \tau(\%)^{\mathrm{e}}}$ & W. Av. $\tau^{\mathrm{f}}$ & FRET Eff. $^{\mathrm{g}}$ \\
\hline gQD & $17.1 \mathrm{~ns}$ & - & $14.6 \mathrm{~ns}(36 \%)$ & $19.2 \mathrm{~ns} \mathrm{(64 \% )}$ & $17.5 \mathrm{~ns}$ & - \\
\hline $1: 0.1$ & $13.7 \mathrm{~ns}$ & $20 \%$ & $10.1 \mathrm{~ns}(41 \%)$ & $14.3 \mathrm{~ns} \mathrm{(59 \% )}$ & $12.6 \mathrm{~ns}$ & $28 \%$ \\
\hline $1: 0.5$ & $11.4 \mathrm{~ns}$ & $33 \%$ & $9.0 \mathrm{~ns}(55 \%)$ & $11.7 \mathrm{~ns} \mathrm{(45 \% )}$ & $10.2 \mathrm{~ns}$ & $42 \%$ \\
\hline $1: 5$ & $8.1 \mathrm{~ns}$ & $53 \%$ & $7.0 \mathrm{~ns}(74 \%)$ & $15.6 \mathrm{~ns}(26 \%)$ & $9.3 \mathrm{~ns}$ & $47 \%$ \\
\hline
\end{tabular}

a mol ratio for conjugation of Cy3 to gQD (gQD:Cy3), b lifetime of QDs using the monoexponential decay fit, c FRET efficiency determined using Mono. $\tau$, ${ }^{\mathrm{d}}$ decay time for QDs (short) using the bi-exponential decay fit (relative contribution), e decay time for QDs (long) using the bi-exponential decay fit (relative contribution), ${ }^{f}$ weighted average lifetime for QDs using the short and long decay times from the bi-exponential decay fit, g FRET efficiency determined using the weighted average $\tau$. 


\section{Dye Loading Capacity}

The FRET efficiency for gQD-Cy3 conjugates assumes a centrosymmetric arrangement of " $a$ " number of equivalent acceptors, around a single donor at a defined distance or $r$, seer Equation S8. This calculation also assumes that all the FRET acceptors are at the same defined distance away from the donor, and that the donor emission is from the centre of the NP core. The $r$ value used in these calculations were obtained by adding the radius of the QDs $(\approx 2.7 \mathrm{~nm}$; page S21) with the approximate size of the polymer coating as determined by DLS (avg HD of polymer is 7-10 $\mathrm{nm}$ yielding a radius of $\approx 3.5-5.0 \mathrm{~nm}$ ). This results in an average $r$ value of 6.1 $7.7 \mathrm{~nm}$. The Förster distance used in this calculation was $6.4 \mathrm{~nm}$ and $E_{\text {FRET }}$ values from Table S5 were used to determine the approximate number of FRET acceptors per conjugation ratio, summary in Table S6. Note that both $E_{F R E T}$ from the mono-exponential and bi-exponential decay fits are used in this set of calculations.

Equation S8

$$
E_{F R E T} \approx \frac{a R_{0}^{6}}{r^{6}+a R_{0}^{6}}
$$

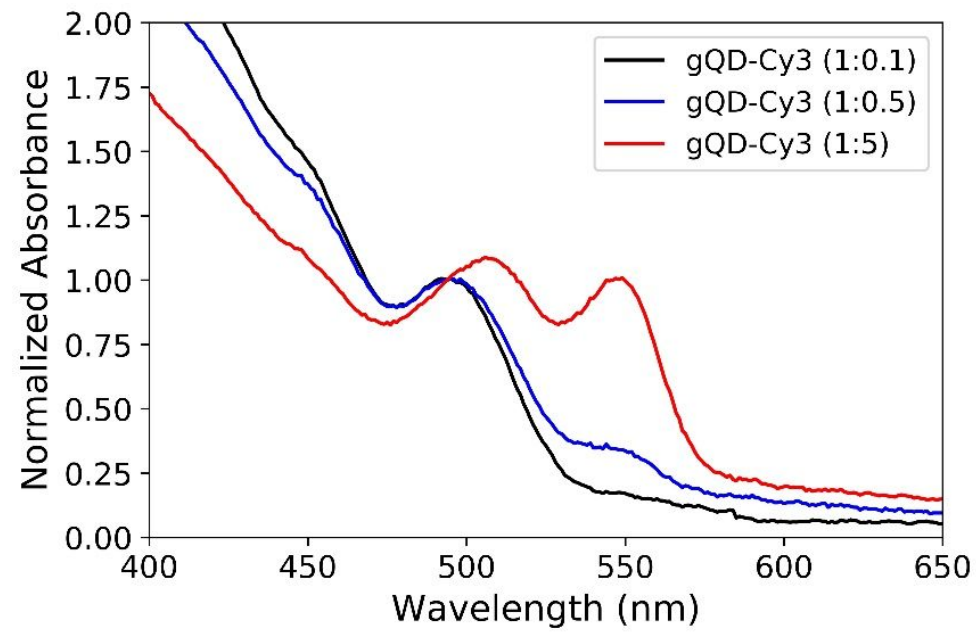

Figure S14 shows normalized absorbance profiles used for calculating the average number of Cy3 conjugated to QDs. The black trace represents gQDs-Cy3 prepared at a conjugation ratio of 1:0.1, 
the blue trace represents gQDs-Cy3 prepared at a conjugation ratio of 1:0.5, and the red trace represents gQDs-Cy3 prepared at a conjugation ratio of 1:5. From spectral analysis, the concentration ratio of gQDs-Cy3 was found to be $0.23,0.53$, and 2.0 Cy3 molecules per QD for the different conditions.

Table S6 Determination of Luminescence Decay Times and Associated FRET efficiencies for QDs coated with BPAOH-PIMA-PEG-N $\mathrm{N}_{3}$ and Conjugated with DBCO-Cy3.

\begin{tabular}{|l|l|l|l|l|l|l|}
\hline Sample $^{\mathrm{a}}$ & FRET Eff. $^{\mathrm{b}}$ & $\begin{array}{l}\text { FRET. D-A. } \\
\text { Sep. UV }\end{array}$ & $\begin{array}{l}\text { \# FRET. A. } \\
\text { T. }^{\mathrm{d}}\end{array}$ & FRET Eff. $^{\text {E }}$ & $\begin{array}{l}\text { FRET. D-A. } \\
\text { Sep. UV }\end{array}$ & $\begin{array}{l}\text { \# FRET. A. } \\
\text { T.g }\end{array}$ \\
\hline $1: 0.1$ & $20 \%$ & $\begin{array}{l}6.3 \mathrm{~nm} \\
(0.23)\end{array}$ & $0.19(0.76)$ & $28 \%$ & $\begin{array}{l}5.8 \mathrm{~nm} \\
(0.23)\end{array}$ & $0.29(1.2)$ \\
\hline $1: 0.5$ & $33 \%$ & $\begin{array}{l}6.5 \mathrm{~nm} \\
(0.53)\end{array}$ & $0.37(1.5)$ & $42 \%$ & $\begin{array}{l}6.1 \mathrm{~nm} \\
(0.53)\end{array}$ & $0.54(2.2)$ \\
\hline $1: 5$ & $53 \%$ & $\begin{array}{l}7.0 \mathrm{~nm} \\
(2.0)\end{array}$ & $0.85(3.4)$ & $47 \%$ & $\begin{array}{l}7.4 \mathrm{~nm} \\
(2.0)\end{array}$ & $0.67(2.7)$ \\
\hline
\end{tabular}

a mol ratio for conjugation of Cy3 to gQD (gQD:Cy3), b FRET Efficiency for QDs as determined in Table S5 using the mono-exponential decay fit, c Experimental FRET donor-acceptor separation distances using mono-exponential FRET efficiencies (number of Cy3 per QD from UV-vis), d number of FRET acceptors assuming that the donor-acceptor separation (D-A) is fixed at $6.1 \mathrm{~nm}$ (D-A = $7.7 \mathrm{~nm}$ ); using mono-functional fit, e FRET Efficiency for QDs as determined in Table S5 using the bi-exponential decay fit, ${ }^{f}$ Experimental FRET donor-acceptor separation distances using bi-exponential FRET efficiencies (number of Cy3 per QD from UV-vis), g number of FRET acceptors assuming that the donor-acceptor separation (D-A) is fixed at $6.1 \mathrm{~nm}(D-A=7.7 \mathrm{~nm})$; using bifunctional fit. Note that mono-exponential decay data is provided for reference but bi-functional is more accurate.

From Table S6, the number of Cy3 dyes loaded onto QDs was found to be from $0.19-3.4$ for mono-exponential decay fit and $0.29-2.7$ for bi-exponential decay fits; depending on the loading conditions. Additionally, the donor-acceptor separation was approximated for varying values of " $a$ ", as determined by UV-vis, and FRET efficiencies. The range of donor-acceptor separation values was found to be $6.3-7.0 \mathrm{~nm}$ for mono-exponential fits and $5.8-7.4 \mathrm{~nm}$ for bi-exponential fits. These values are in good agreement with the previously determined theoretical values for $\mathrm{D}-\mathrm{A}$ separation $(6.1-7.7 \mathrm{~nm})$ and suggest a theoretical dye loading capacity of 2.7 per QD. 
14.Additional Information (1H, 13C, 31P NMR, FTIR, and LC-MS-MS Data)

\section{Table of Contents}

14.1. Data for mPEG-OH (MW of $750 \mathrm{~g} / \mathrm{mol}$ ) derivatives; $1 \mathrm{H}$ NMR, ATR-FTIR ...............................34

14.2. Data for HO-PEG-OH (MW of $600 \mathrm{~g} / \mathrm{mol}$ ) derivatives; $1 \mathrm{H}$ NMR, ATR-FTIR ...............................36

14.3. Data for BOC amine protected mono-phosphonate ester [NHBOC-PAOEt]; 1H NMR, 13C NMR, 31P NMR, LC-MS/MS

14.4. Data for BOC deprotection of mono-phosphonate ester [ $\left.\mathrm{NH}_{2}-\mathrm{PAOEt}\right]$; $1 \mathrm{H}$ NMR, $13 \mathrm{C}$ NMR, 31P NMR, LC-MS/MS.

14.5. Data for BOC amine protected bis-phosphonate ester [NHBOC-(PAOEt) ${ }_{2}$ ]; 1 H NMR, $13 C$ NMR, 31P NMR, LC-MS/MS

14.6. Data for BOC deprotected bis-phosphonate ester $\left[\mathrm{NH}_{2}-(\mathrm{PAOEt})_{2}\right] ; 1 \mathrm{H} \mathrm{NMR}, 13 \mathrm{C} \mathrm{NMR}, 31 \mathrm{P}$ NMR, LC-MS/MS.

14.7. Data for MPAOEt-PIMA-PEG co-polymers (grafting ratio 25:75); 1H NMR, 31P NMR, ATR-FTIR

14.8. Data for MPAOEt-PIMA-PEG co-polymers (grafting ratio 50:50); 1H NMR, 31P NMR, ATR-FTIR

14.9. Data for BPAOEt-PIMA-PEG co-polymers (grafting ratio 25:75); 1H NMR, 31P NMR, ATR-FTIR... 50

14.10. Data for BPAOEt-PIMA-PEG co-polymers (grafting ratio 50:50); 1H NMR, 31P NMR, ATR-FTIR

14.11. Data for BPAOEt-PIMA-PEG-N ${ }_{3}$ co-polymers (grafting ratio 50:45:5); 1 H NMR, 31P NMR, ATR-FTIR.

14.12. Data for MPAOH-PIMA-PEG co-polymers (grafting ratio 25:75); $1 \mathrm{H}$ NMR, 31P NMR, ATRFTIR.

14.13. Data for MPAOH-PIMA-PEG co-polymers (grafting ratio 50:50); $1 \mathrm{H}$ NMR, 31P NMR, ATRFTIR.

14.14. Data for BPAOH-PIMA-PEG co-polymers (grafting ratio 25:75); 1H NMR, 31P NMR, ATRFTIR.

14.15. Data for BPAOH-PIMA-PEG co-polymers (grafting ratio 50:50); 1H NMR, 31P NMR, ATRFTIR..

14.16. Data for BPAOH-PIMA-PEG-N ${ }_{3}$ co-polymers (grafting ratio 50:45:5); 1 H NMR, 31P NMR, ATR-FTIR. .64

14.17. Summary of Characterization Data for Protected Polymers ............................................64

14.18. Summary of Characterization Data for Final Polymers .64 
14.1 Data for mPEG-OH (MW of $750 \mathrm{~g} / \mathrm{mol}$ ) derivatives

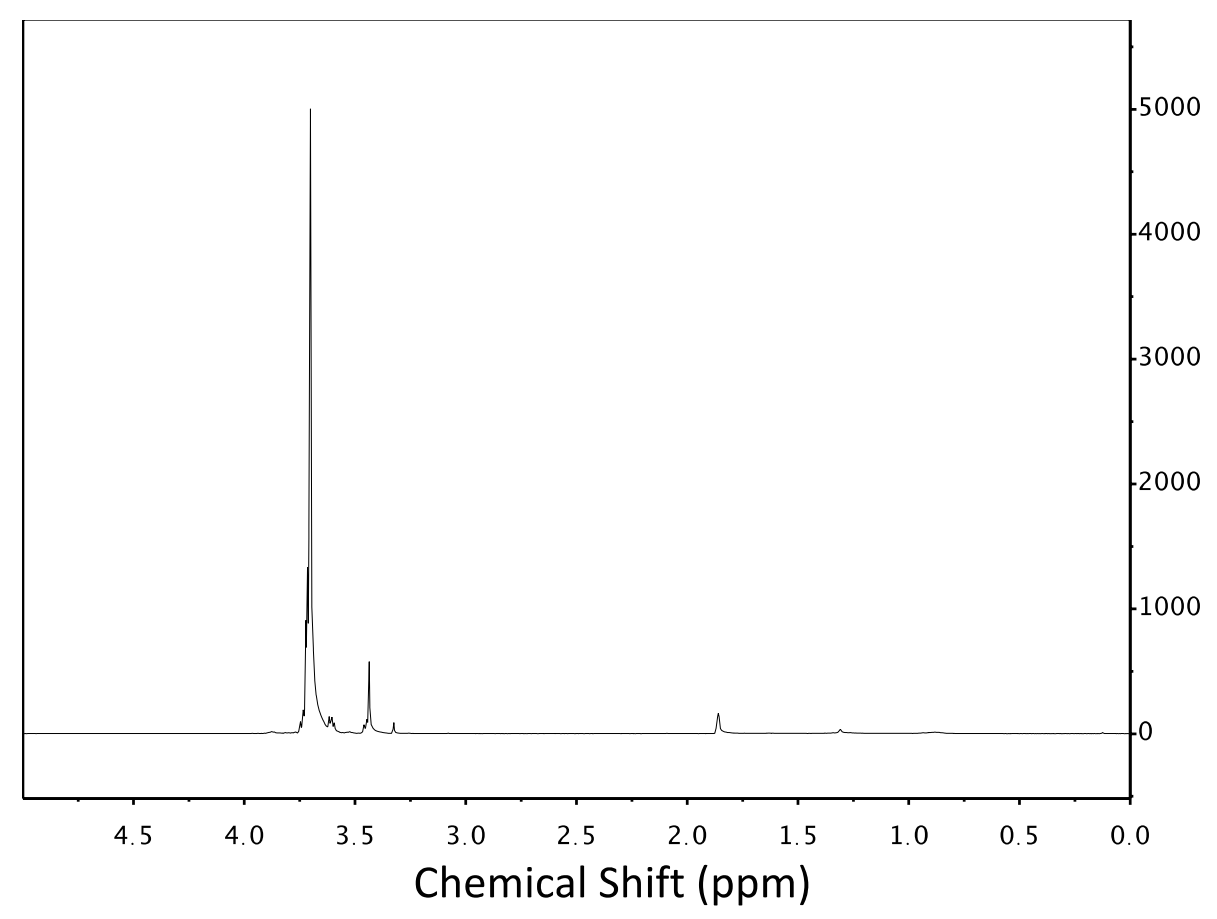

Figure S15. $1 \mathrm{H}$ NMR of $\mathrm{mPEG}-\mathrm{N}_{3}$ in $\mathrm{CDCl}_{3}$.

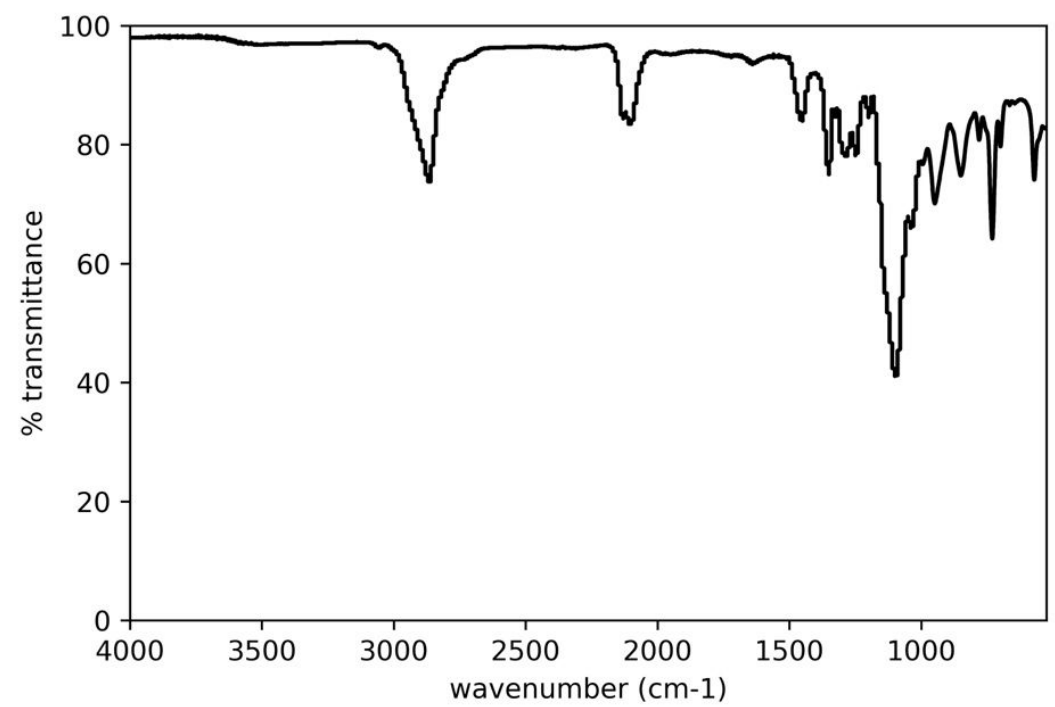

Figure S16. ATR-FTIR data for mPEG-N ${ }_{3}$ with the following peaks of interest: 2866 (C-H stretch), 2103 ( $\mathrm{N}_{3}$ stretch), 1451 (C-H bending), 1349, 1284, 1248, 1097 (C-O stretch), 949, $850 \mathrm{~cm}^{-1}$. 


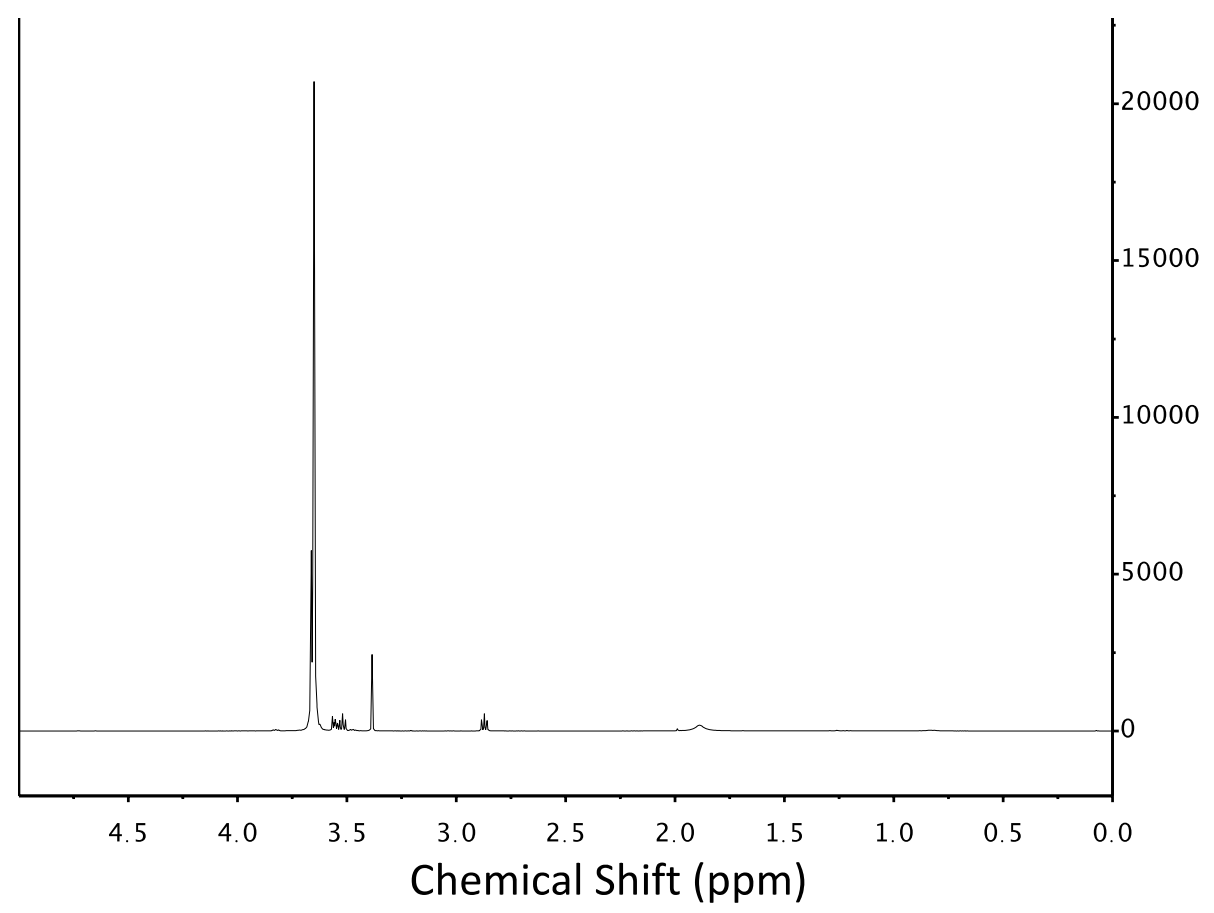

Figure S17. $1 \mathrm{H}$ NMR of mPEG-NH $\mathrm{N}_{2}$ in $\mathrm{CDCl}_{3}$.

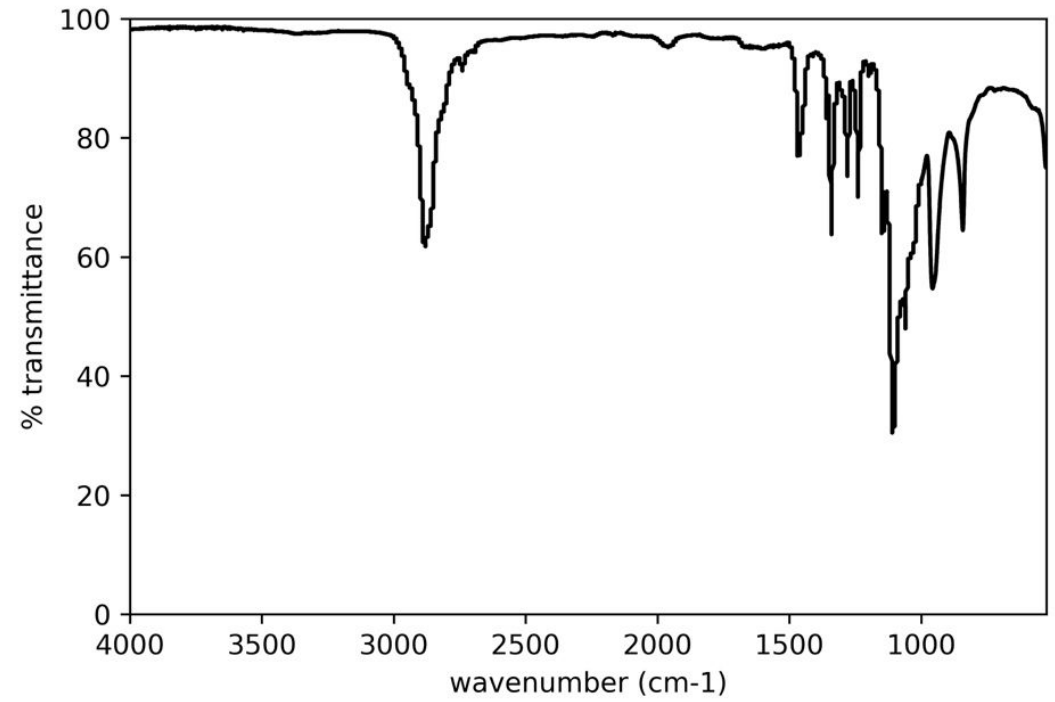

Figure S18. ATR-FTIR data for mPEG-NH 2 with the following peaks of interest: 2882 (C-H stretch), 1465 (C-H bending), 1340, 1280, 1240, 1107 (C-O stretch), 957, $842 \mathrm{~cm}^{-1}$. 


\subsection{Data for HO-PEG-OH (MW of $600 \mathrm{~g} / \mathrm{mol}$ ) derivatives}

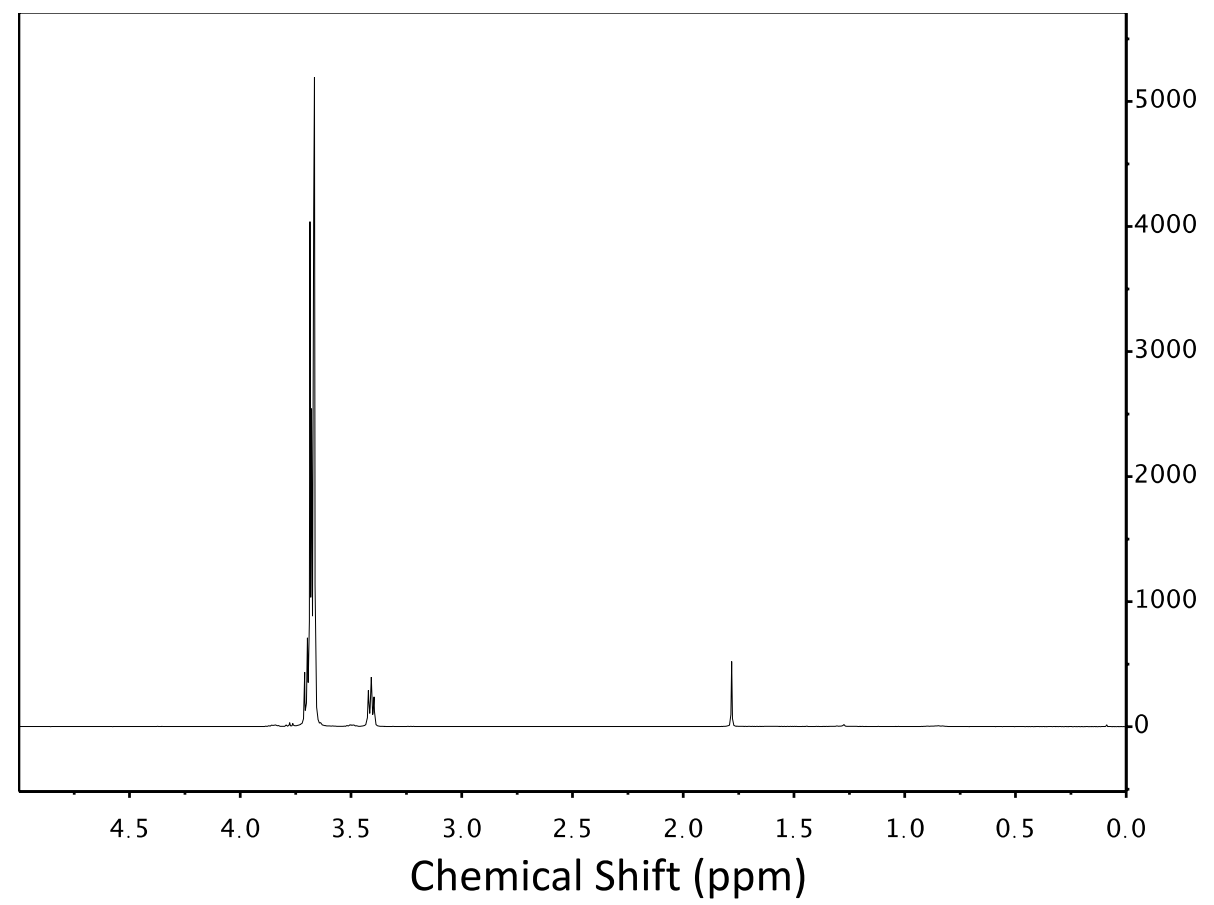

Figure S19. $1 \mathrm{H}$ NMR of $\mathrm{N}_{3}-\mathrm{PEG}-\mathrm{N}_{3}$ in $\mathrm{CDCl}_{3}$.

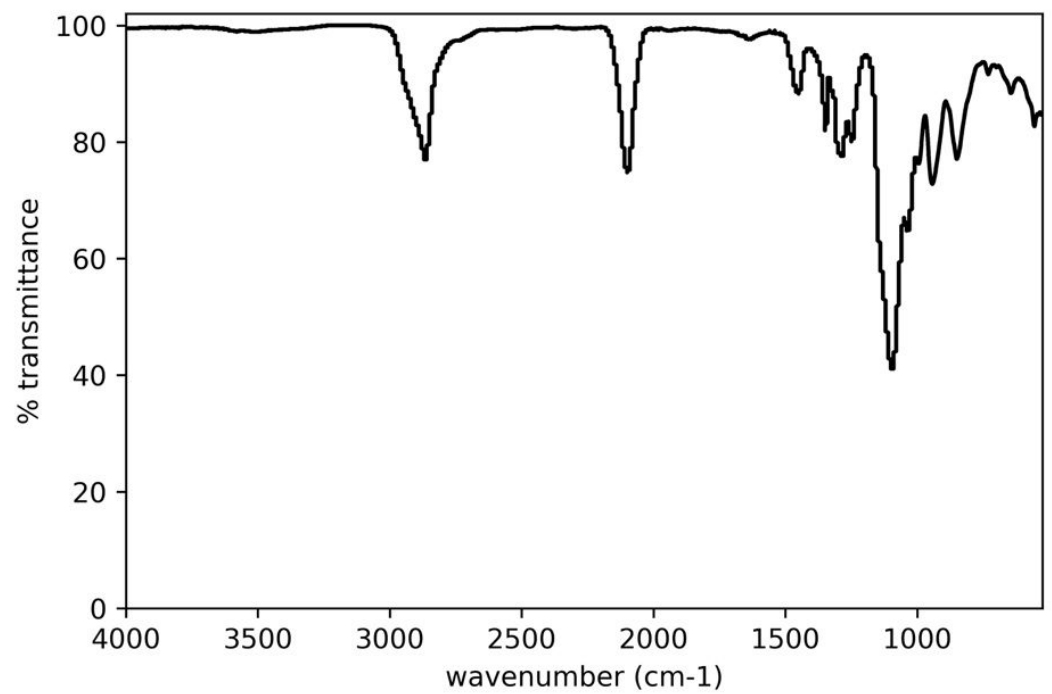

Figure S20. ATR-FTIR data for $\mathrm{N}_{3}-\mathrm{PEG}-\mathrm{N}_{3}$ with the following peaks of interest: 2865 (C-H stretch), 2099 ( $\mathrm{N}_{3}$ stretch), 1450 (C-H bending), 1347, 1285, 1249, 1096 (C-O stretch), 943, $850 \mathrm{~cm}^{-1}$. 


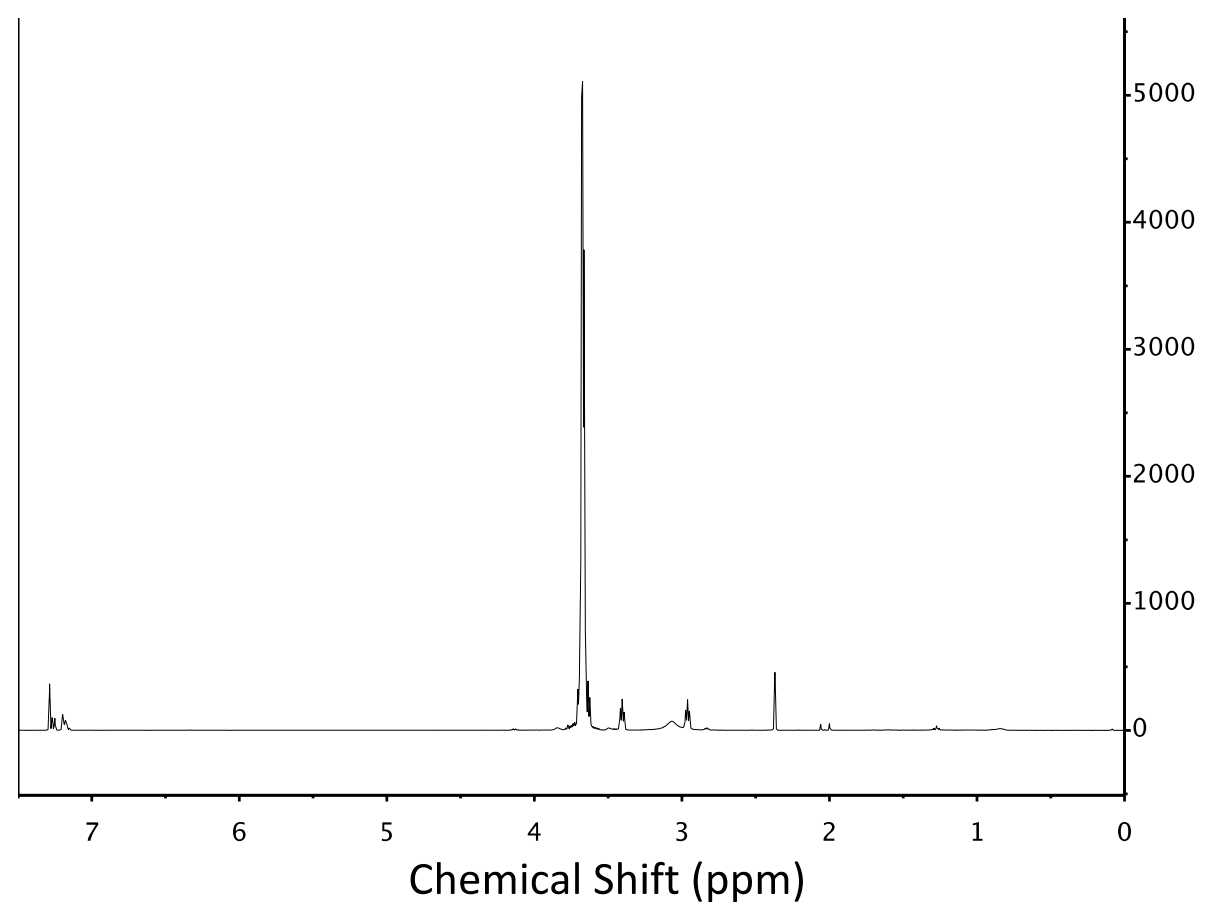

Figure S21. 1H NMR of $\mathrm{NH}_{2}-\mathrm{PEG}-\mathrm{N}_{3}$ in $\mathrm{CDCl}_{3}$.

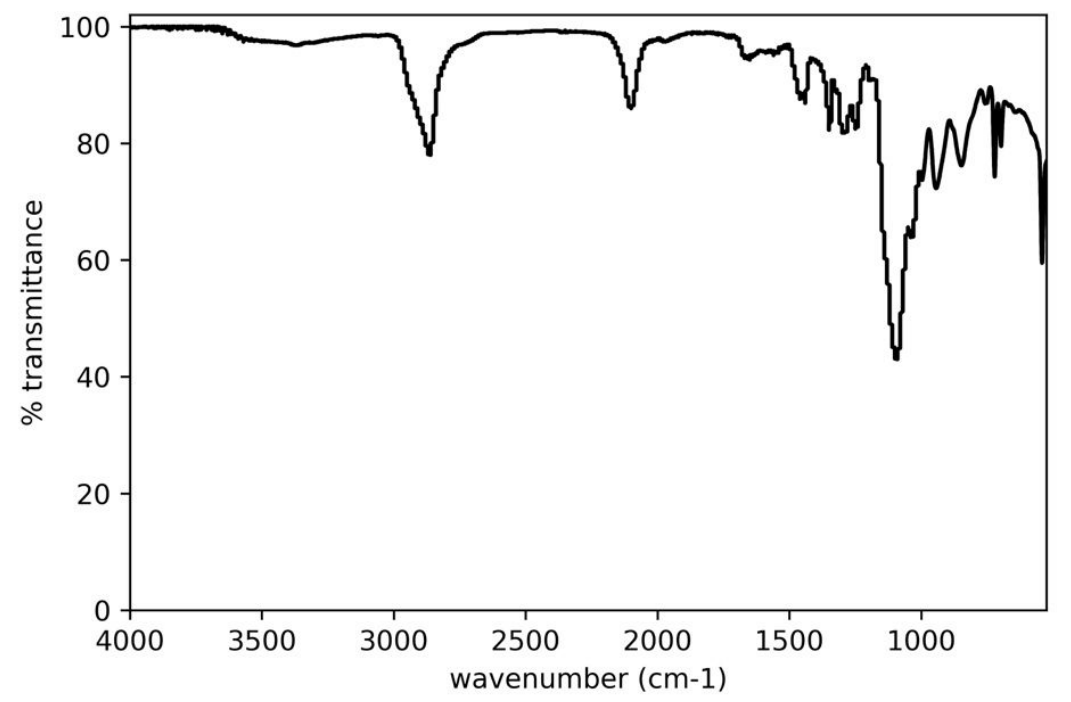

Figure S22. ATR-FTIR data for $\mathrm{NH}_{2}-\mathrm{PEG}-\mathrm{N}_{3}$ with the following peaks of interest: 3366 ( $\mathrm{N}-\mathrm{H}$ stretch), 2865 (C-H stretch), 2100 ( $\mathrm{N}_{3}$ stretch), 1654, 1438 (C-H bending), 1348, 1296, 1248, 1094 (C-O stretch), $944,847 \mathrm{~cm}^{-1}$. 
14.3 Data for BOC amine protected mono-phosphonate ester [NHBOC-PAOEt]

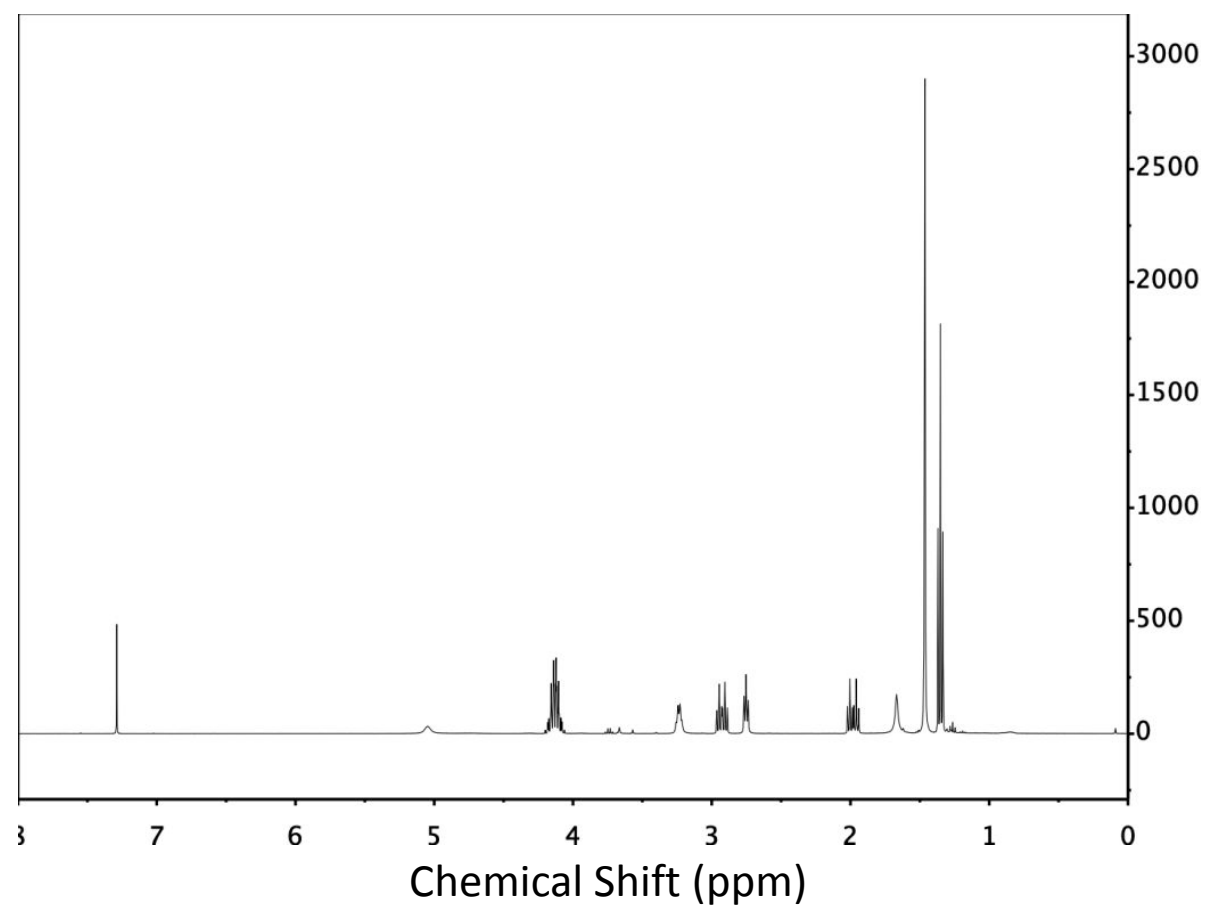

Figure $\mathrm{S} 23.1 \mathrm{H}$ NMR of amine-BOC protected mono-phosphonate ester in $\mathrm{CDCl}_{3}$.

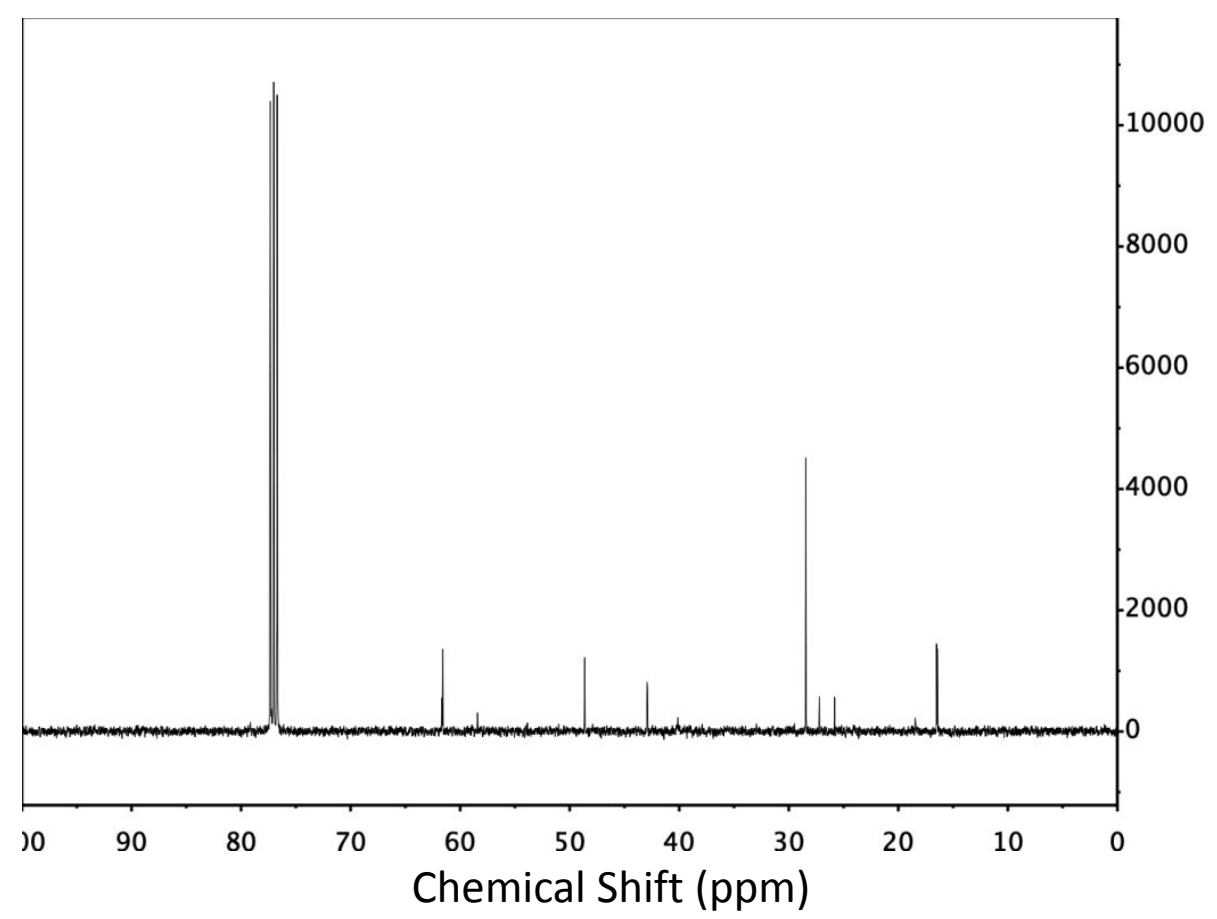

Figure S24. 13C NMR of amine-BOC protected mono-phosphonate ester in $\mathrm{CDCl}_{3}$. 


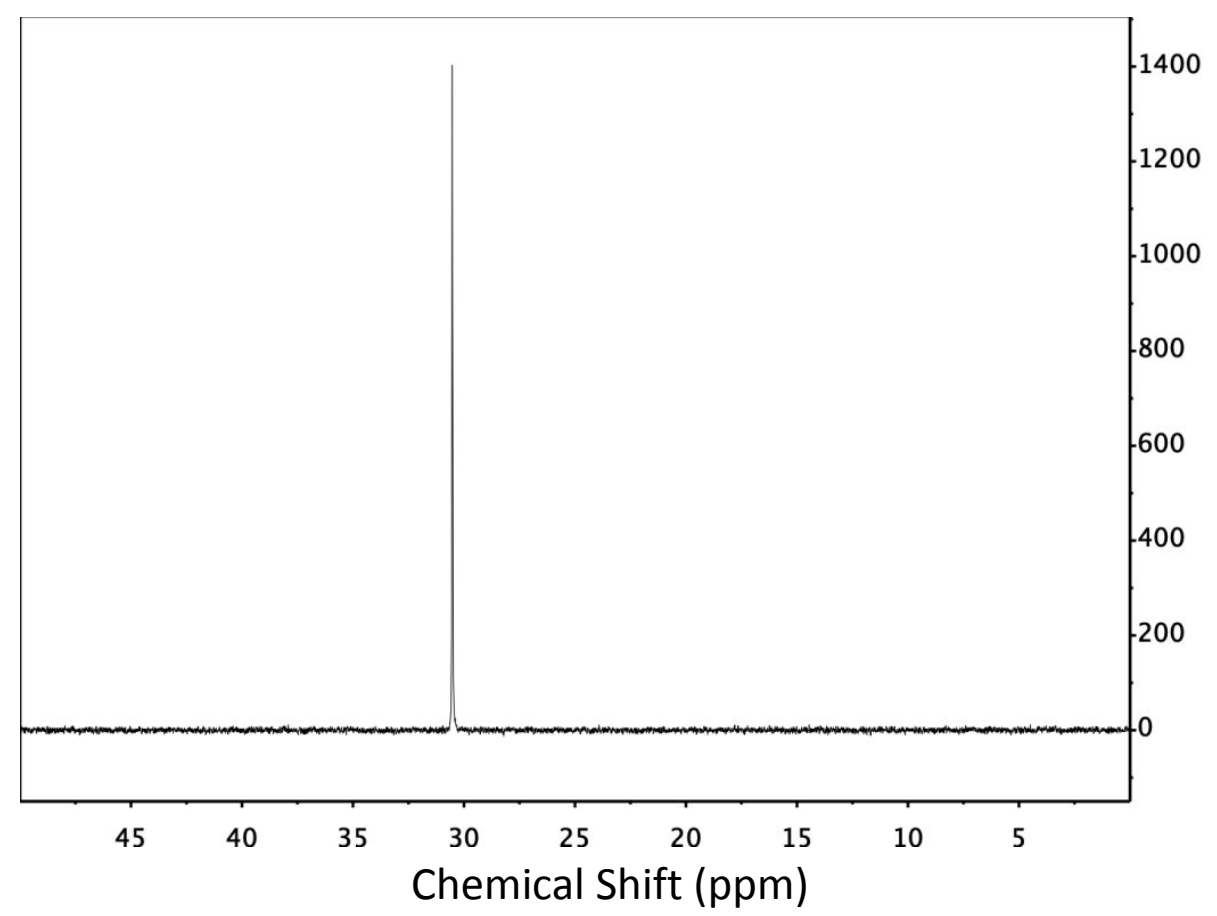

Figure S25. 31P NMR of amine-BOC protected mono-phosphonate ester in $\mathrm{CDCl}_{3}$.

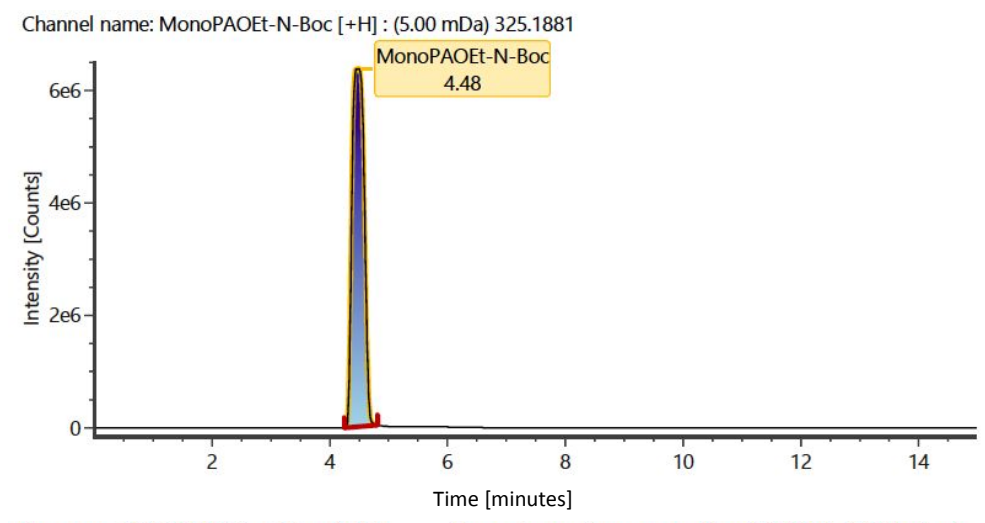

Item name: 20190618_Richard_Sample1_b Channel name: Low energy : Time 4.5350 +/- 0.0451 minutes Item description:

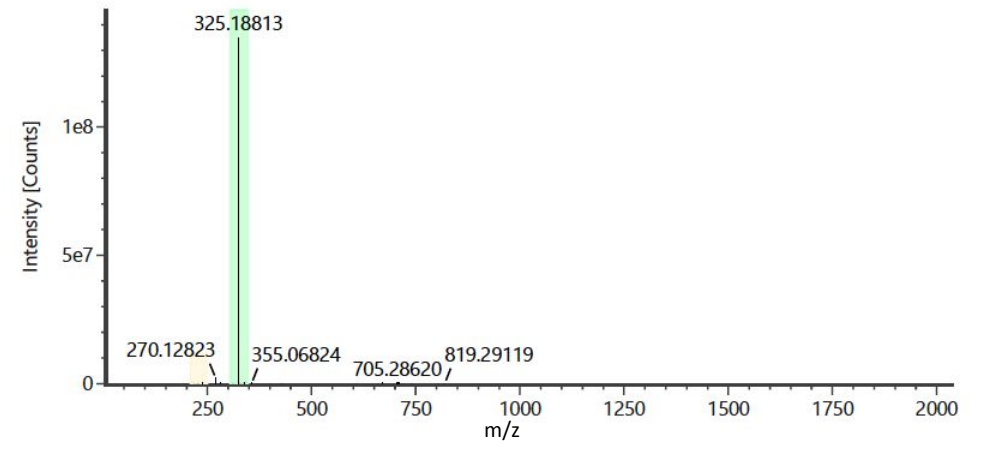

Figure S26. LC-MS/MS for amine-BOC protected mono-phosphonate ester. (Top) LC chromatogram of mono-phosphonate-NHBOC and (Bottom) HR-MS spectra. 
14.4 Data for BOC deprotection of mono-phosphonate ester [ $\left.\mathrm{NH}_{2}-\mathrm{PAOEt}\right]$

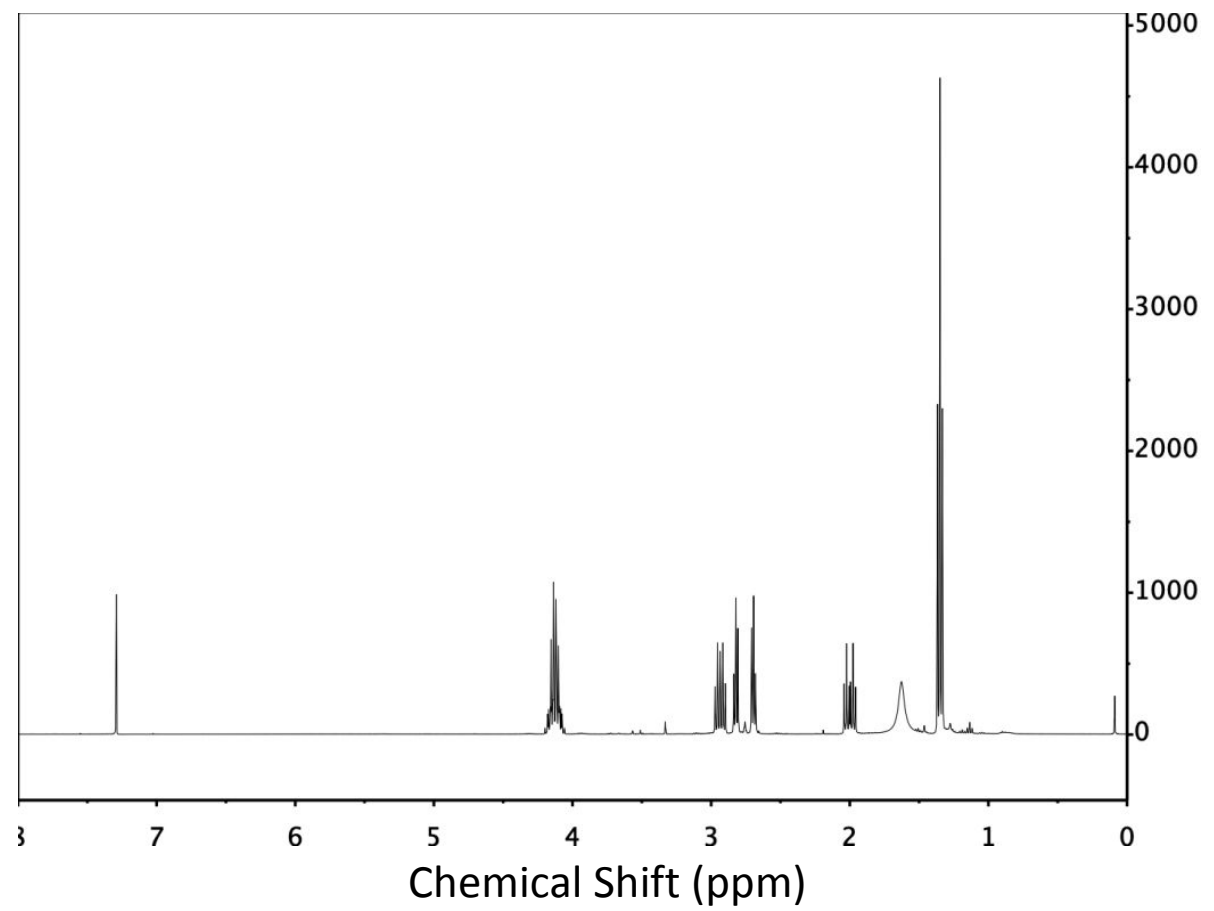

Figure S27. 1H NMR of BOC deprotected mono-phosphonate ester in $\mathrm{CDCl}_{3}$.

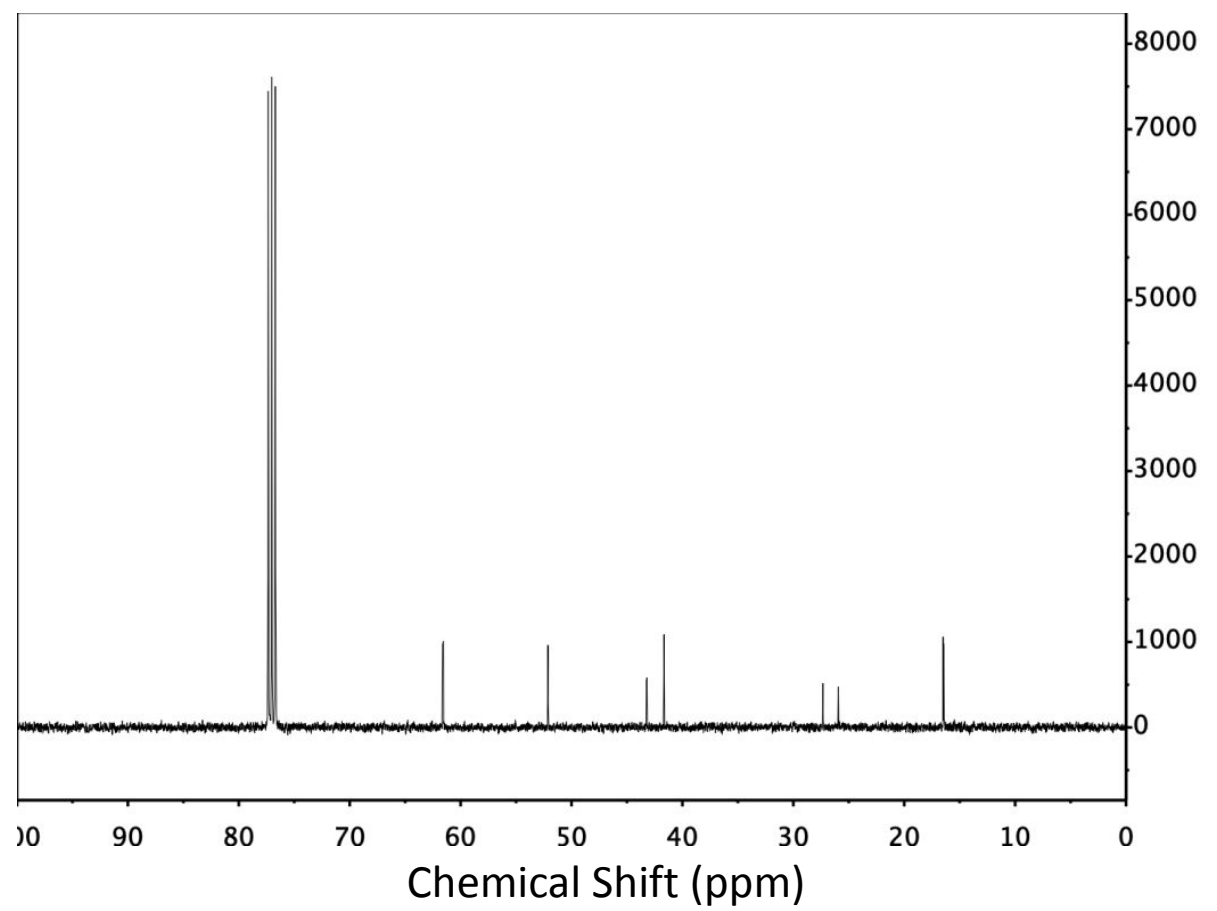

Figure S28. 13C NMR of BOC deprotected mono-phosphonate ester in $\mathrm{CDCl}_{3}$. 


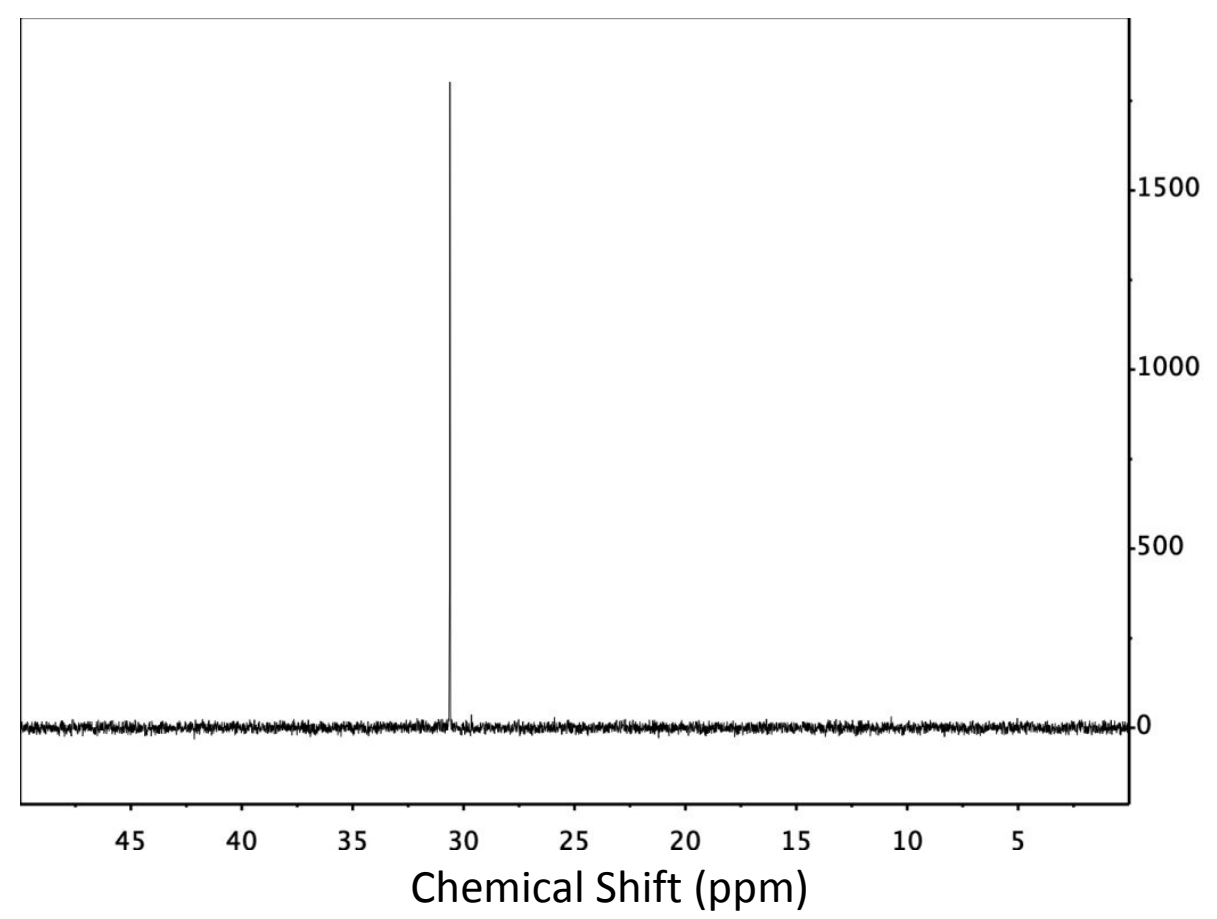

Figure S29. 31P NMR of BOC deprotected mono-phosphonate ester in $\mathrm{CDCl}_{3}$.

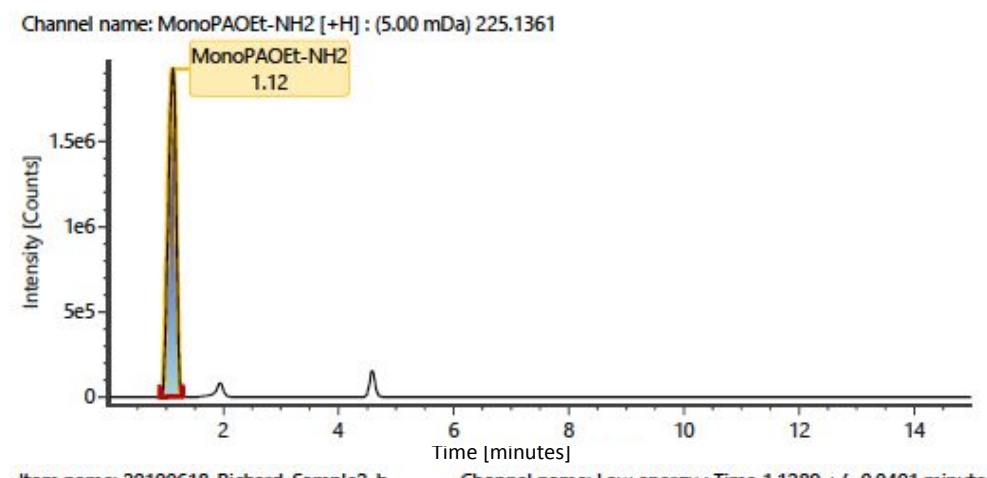

Item name: 20190618_Richard_Sample2_b Channel name: Low energy : Time 1.1289 +/- 0.0401 minutes Item description:

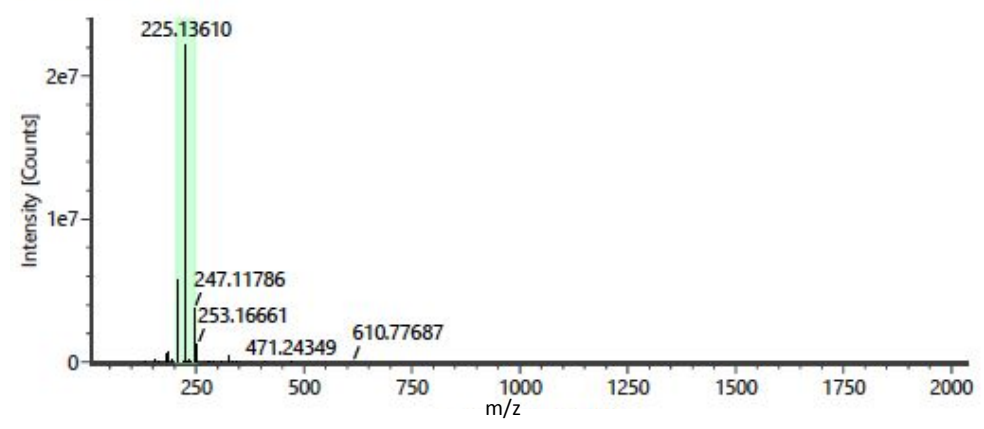

Figure S30. LC-MS/MS for BOC deprotected mono-phosphonate ester. (Top) LC chromatogram of mono-phosphonate- $\mathrm{NH}_{2}$ and (Bottom) HR-MS spectra. 
14.5 Data for BOC amine protected bis-phosphonate ester [NHBOC-(PAOEt $)_{2}$ ]

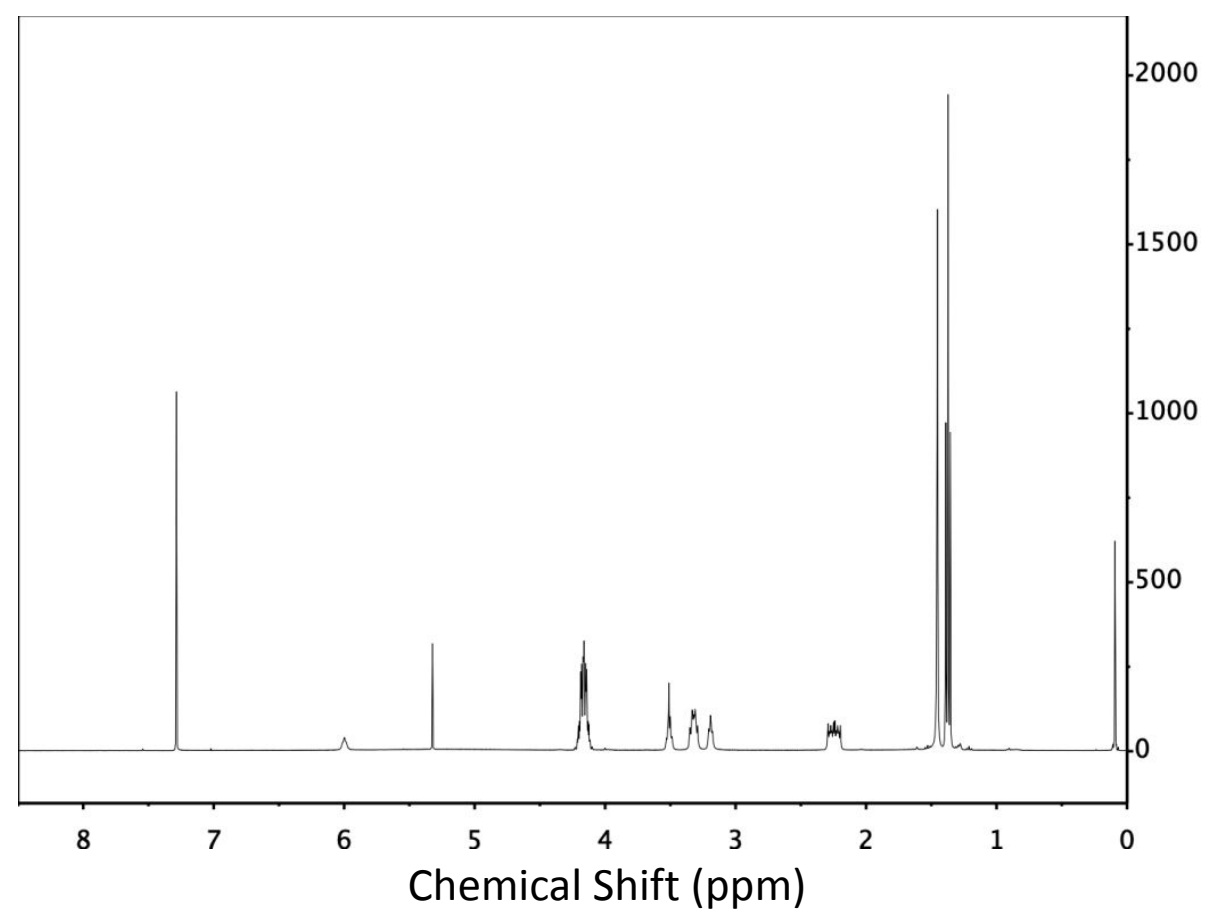

Figure S31. 1H NMR of amine-BOC protected bis-phosphonate ester in $\mathrm{CDCl}_{3}$.

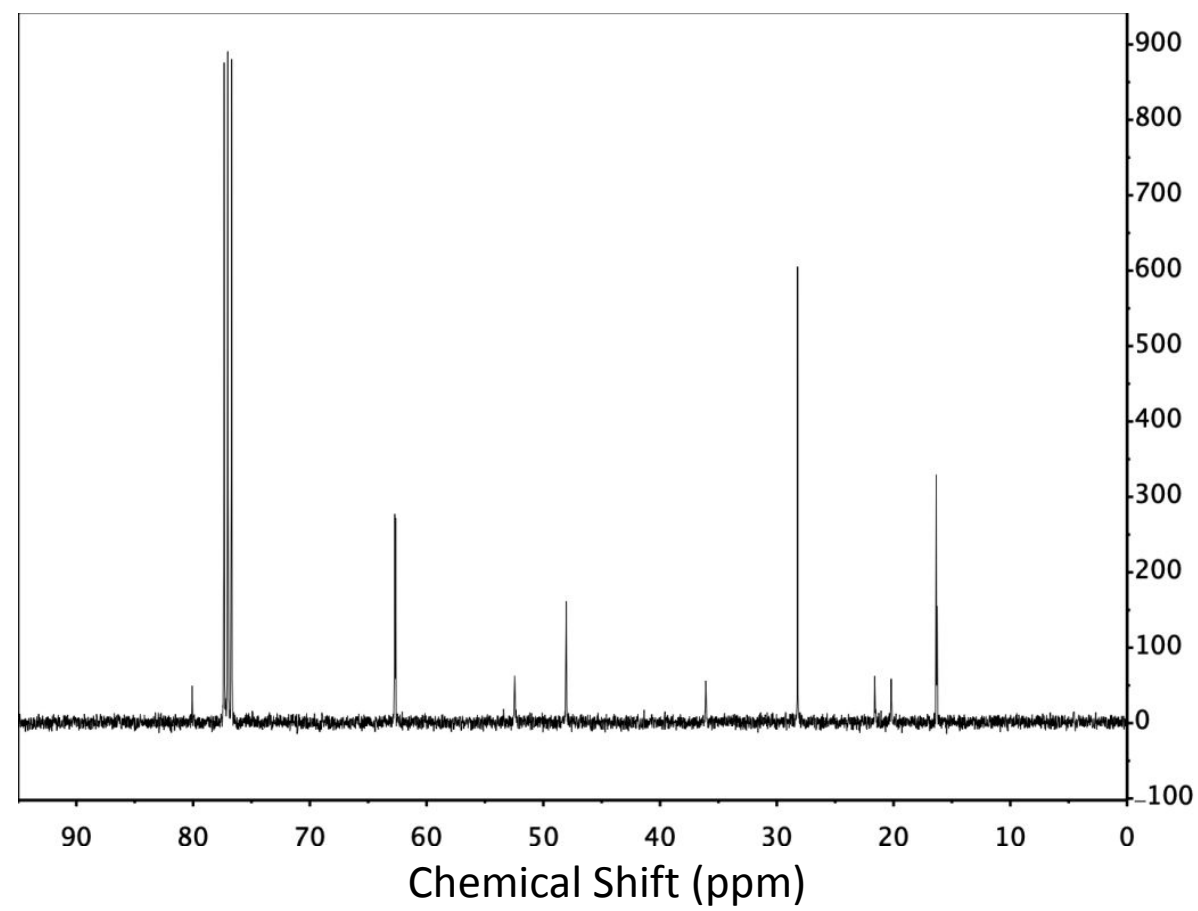

Figure S32. $13 \mathrm{C}$ NMR of amine-BOC protected bis-phosphonate ester in $\mathrm{CDCl}_{3}$. 


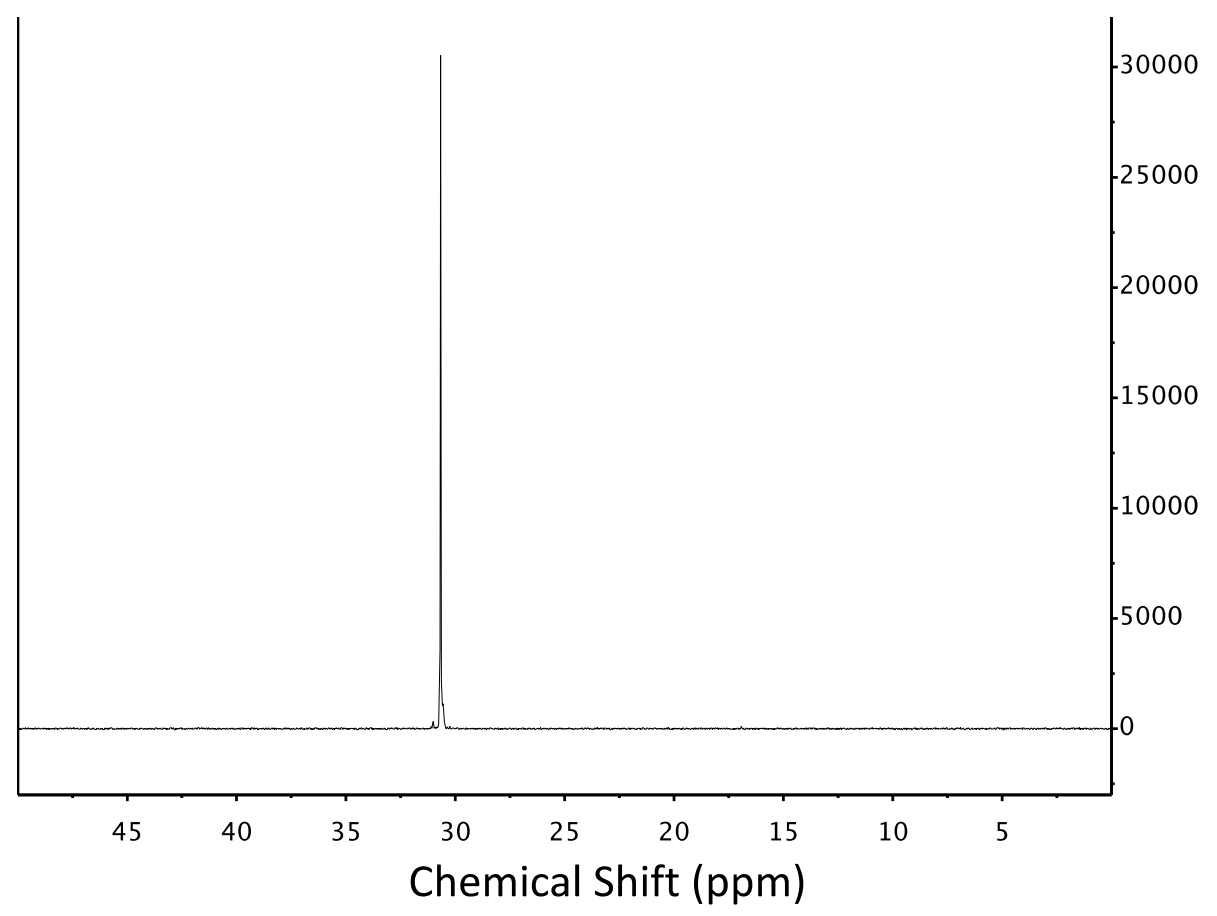

Figure S33. 31P NMR of amine-BOC protected bis-phosphonate ester in $\mathrm{CDCl}_{3}$.

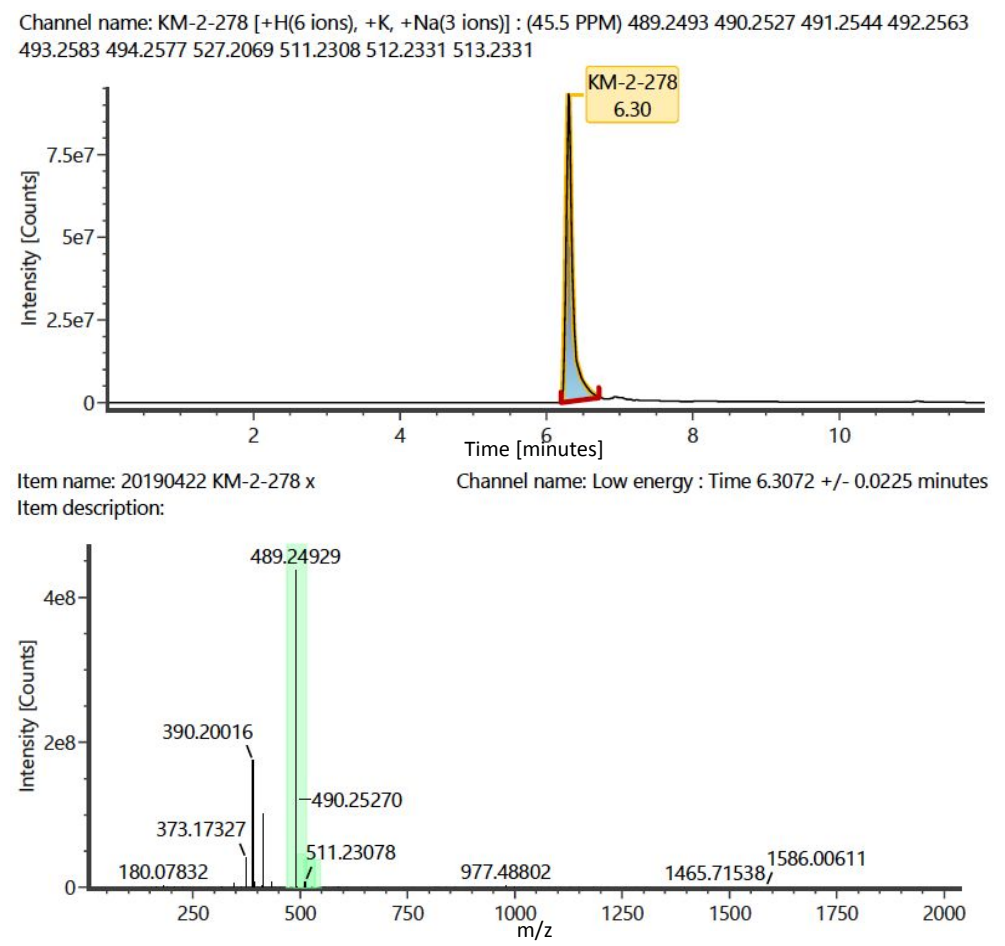

Figure S34. LC-MS/MS for amine-BOC protected bis-phosphonate ester. (Top) LC chromatogram of bis-phosphonate-NHBOC and (Bottom) HR-MS spectra. 
14.6 Data for BOC deprotected bis-phosphonate ester $\left[\mathrm{NH}_{2}-(\mathrm{PAOEt})_{2}\right]$

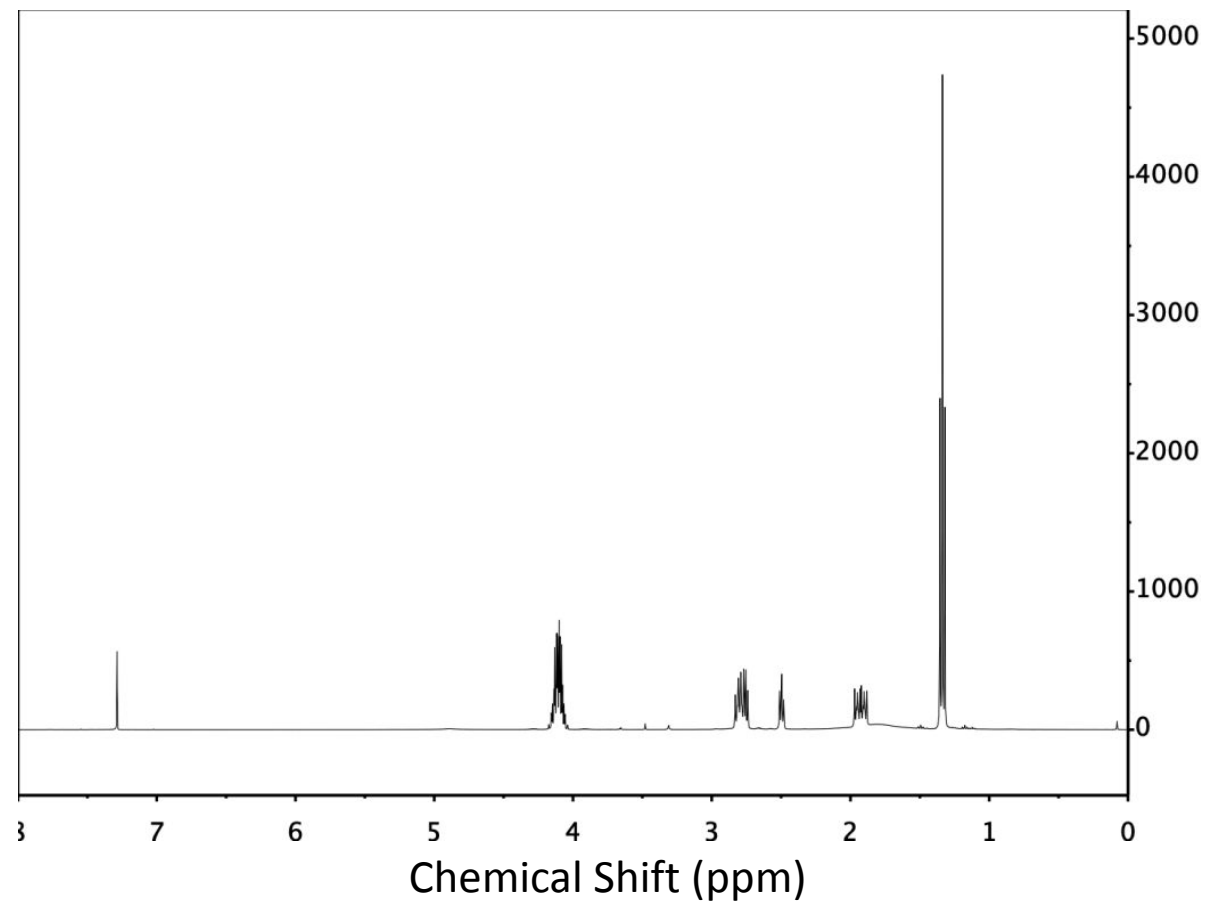

Figure S35. 1H NMR of BOC deprotected bis-phosphonate ester in $\mathrm{CDCl}_{3}$.

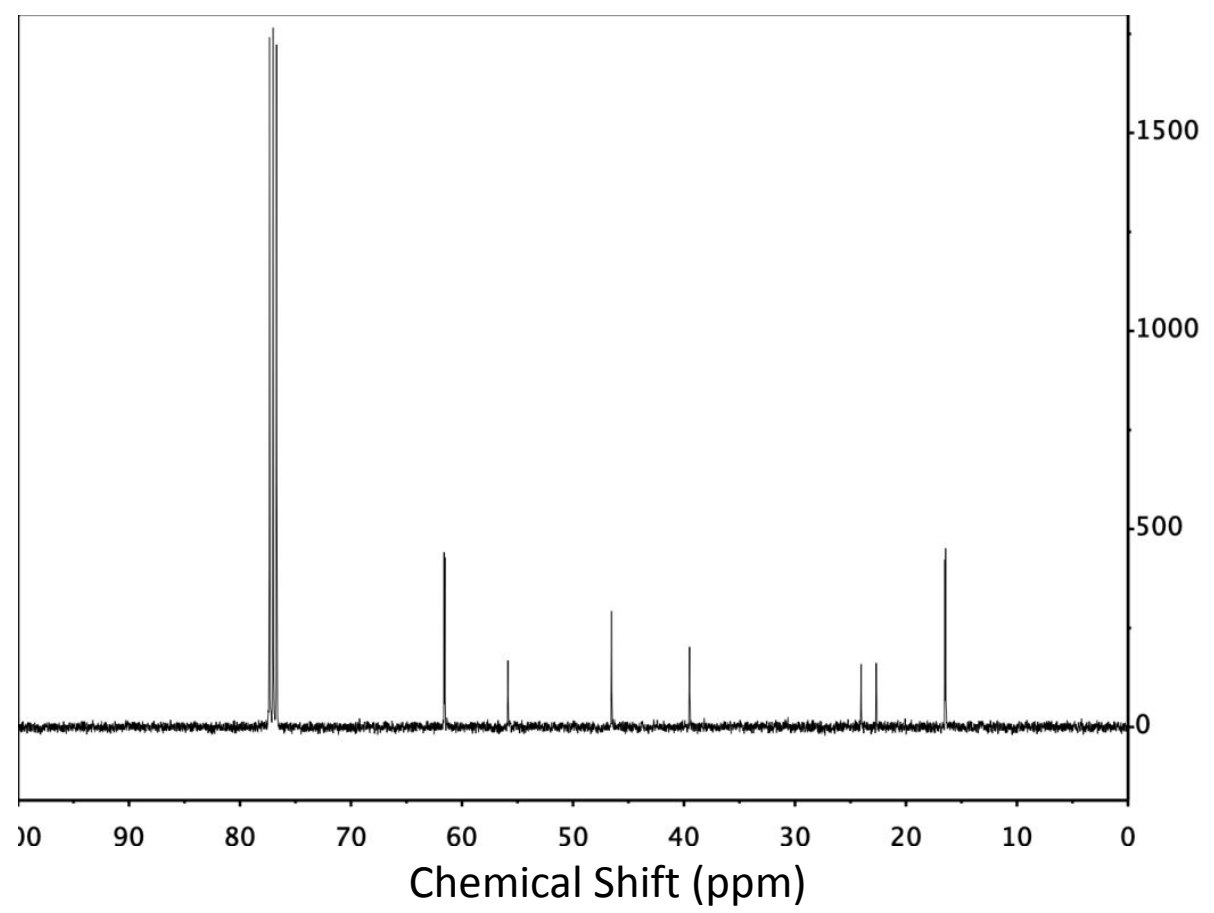

Figure S36. 13C NMR of BOC deprotected bis-phosphonate ester in $\mathrm{CDCl}_{3}$. 


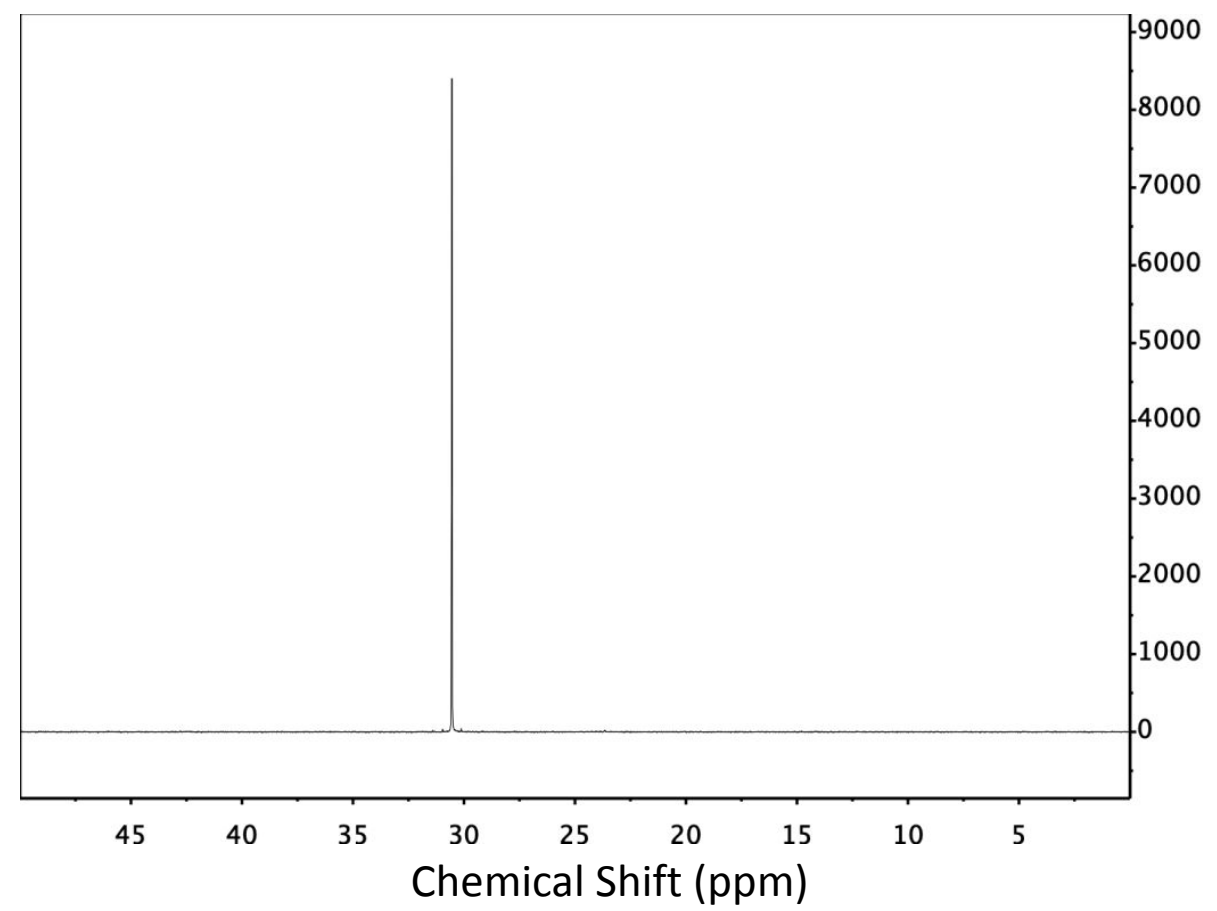

Figure S37. 31P NMR of BOC deprotected bis-phosphonate ester in $\mathrm{CDCl}_{3}$.

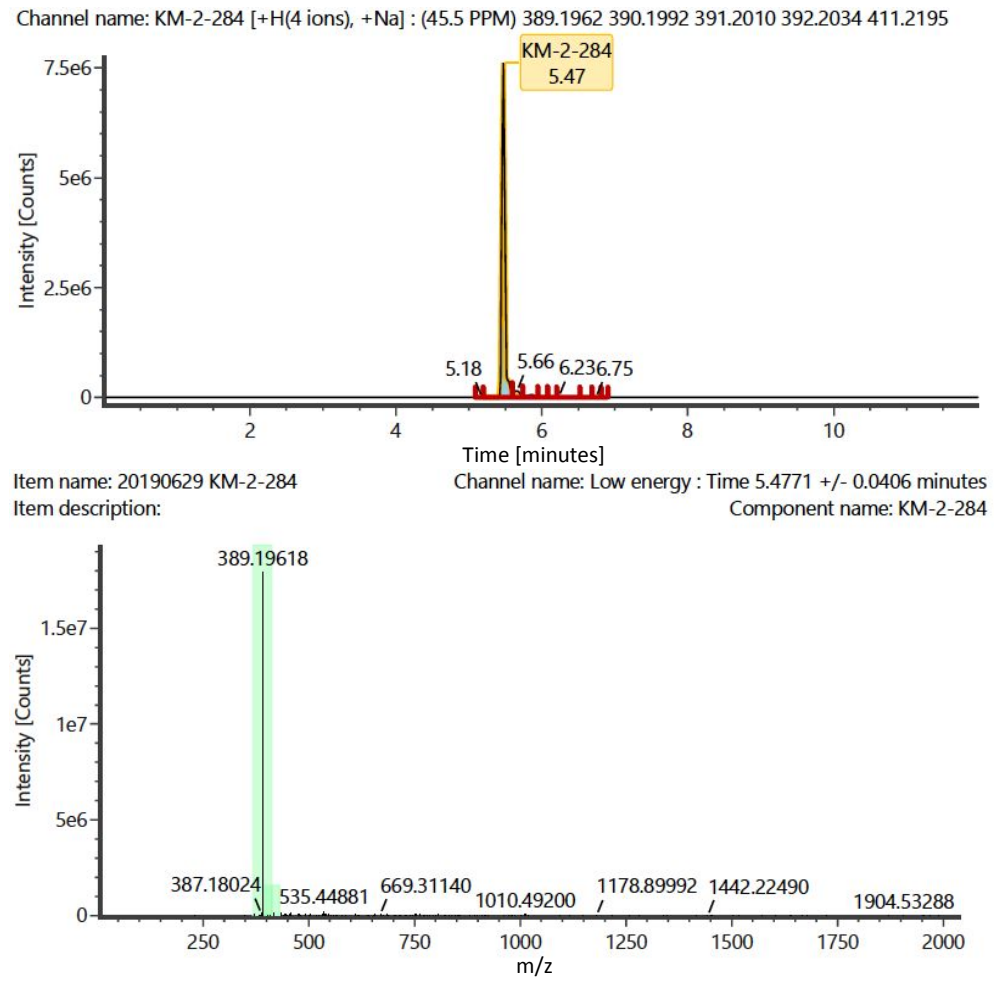

Figure S38. LC-MS/MS for BOC deprotected bis-phosphonate ester. (Top) LC chromatogram of bis-phosphonate- $\mathrm{NH}_{2}$ and (Bottom) HR-MS spectra. 
14.7 Data for MPAOEt-PIMA-PEG co-polymers (grafting ratio 25:75)

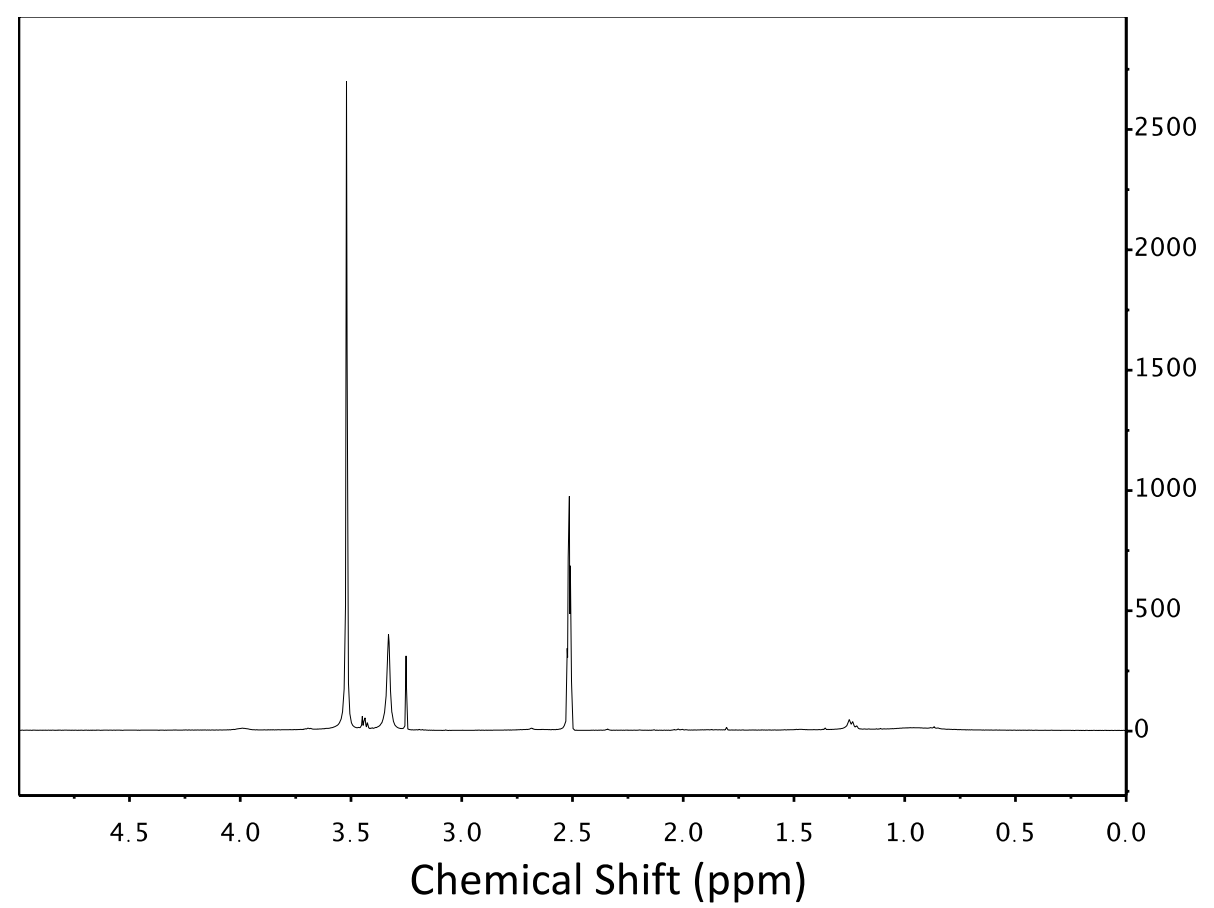

Figure S39. 1H NMR of MPAOEt-PIMA-PEG copolymer (25:75) in DMSO-d6.

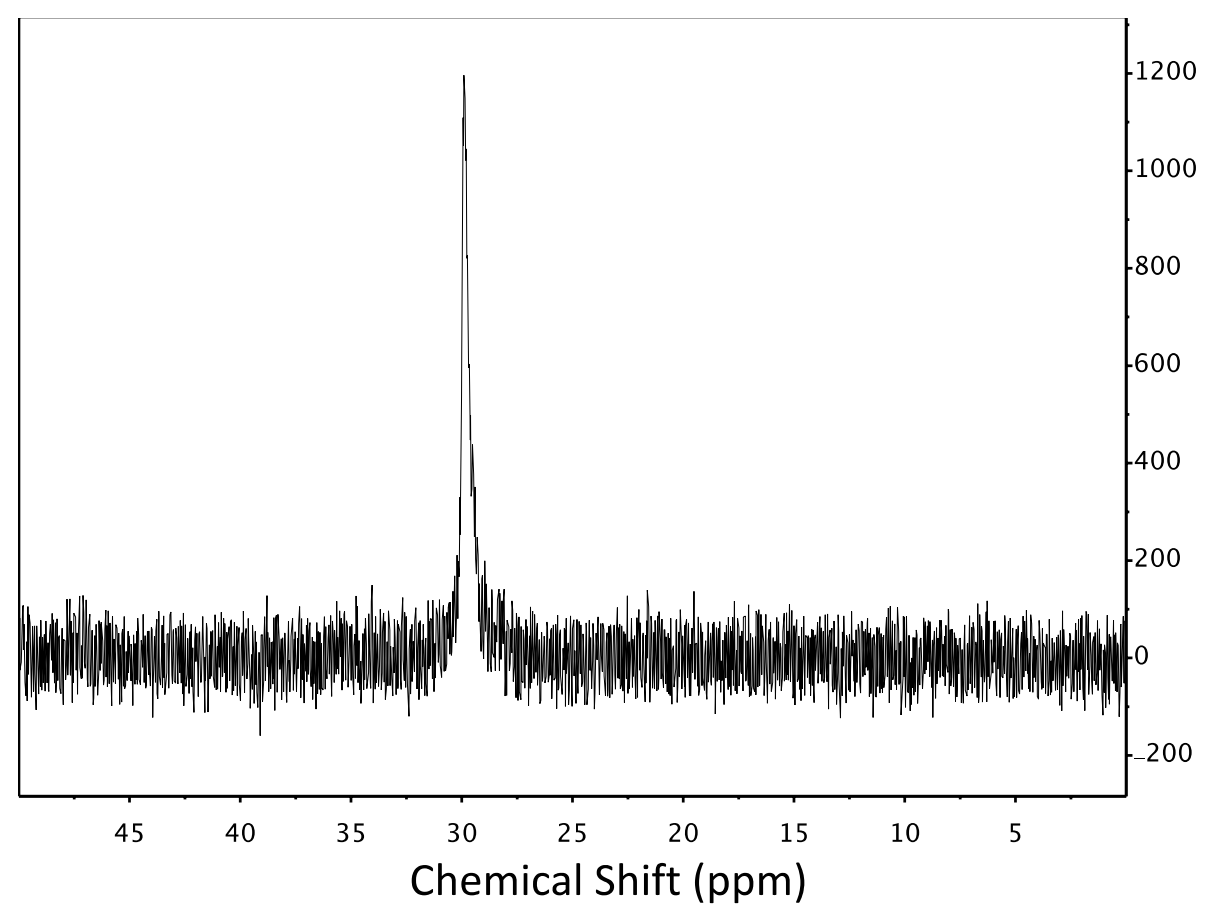

Figure S40. 31P NMR of MPAOEt-PIMA-PEG copolymer (25:75) in DMSO-d6. 


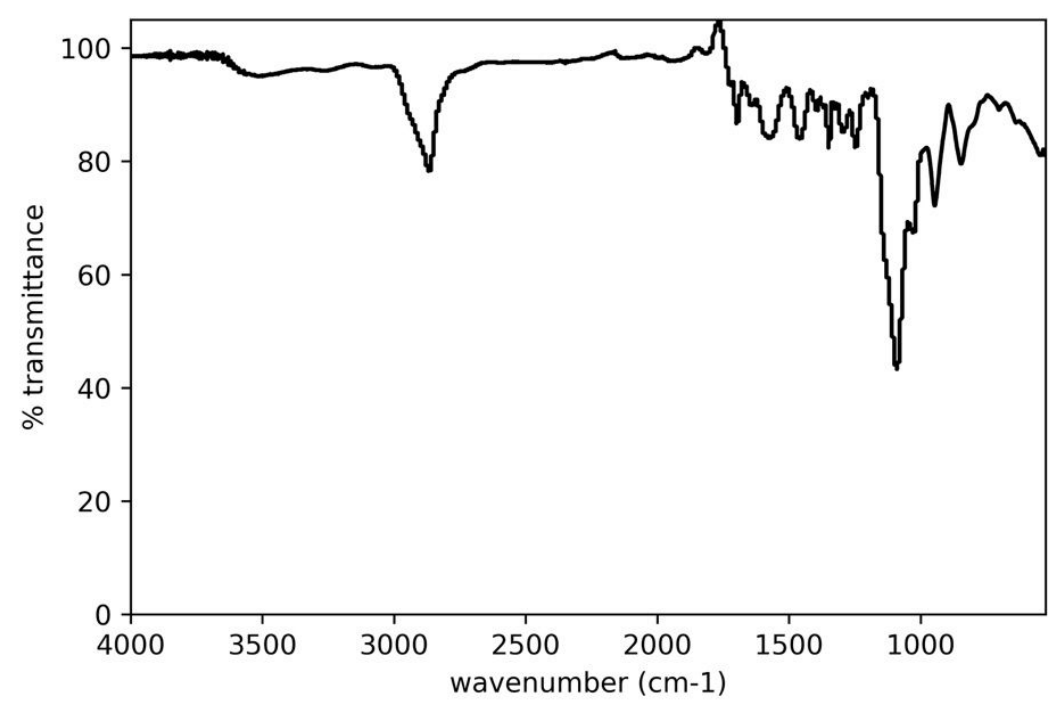

Figure S41. ATR-FTIR data for MPAOEt-PIMA-PEG (25:75) with the following peaks of interest: 3500 and 3286 (O-H stretching, carboxylic acid), 2867 (C-H stretching, alkane), 1697 (C=O stretch, cyclic imide- side product), 1575 (C=O stretching; $2 \circ \mathrm{N}-\mathrm{H}$ bending), 1456, 1394, 1296, 1247, 1091 (C-O-C stretch PEG), 1028 (C-O stretch PAOEt \& PEG), 947, $846 \mathrm{~cm}^{-1}$. 
14.8 Data for MPAOEt-PIMA-PEG co-polymers (grafting ratio 50:50)

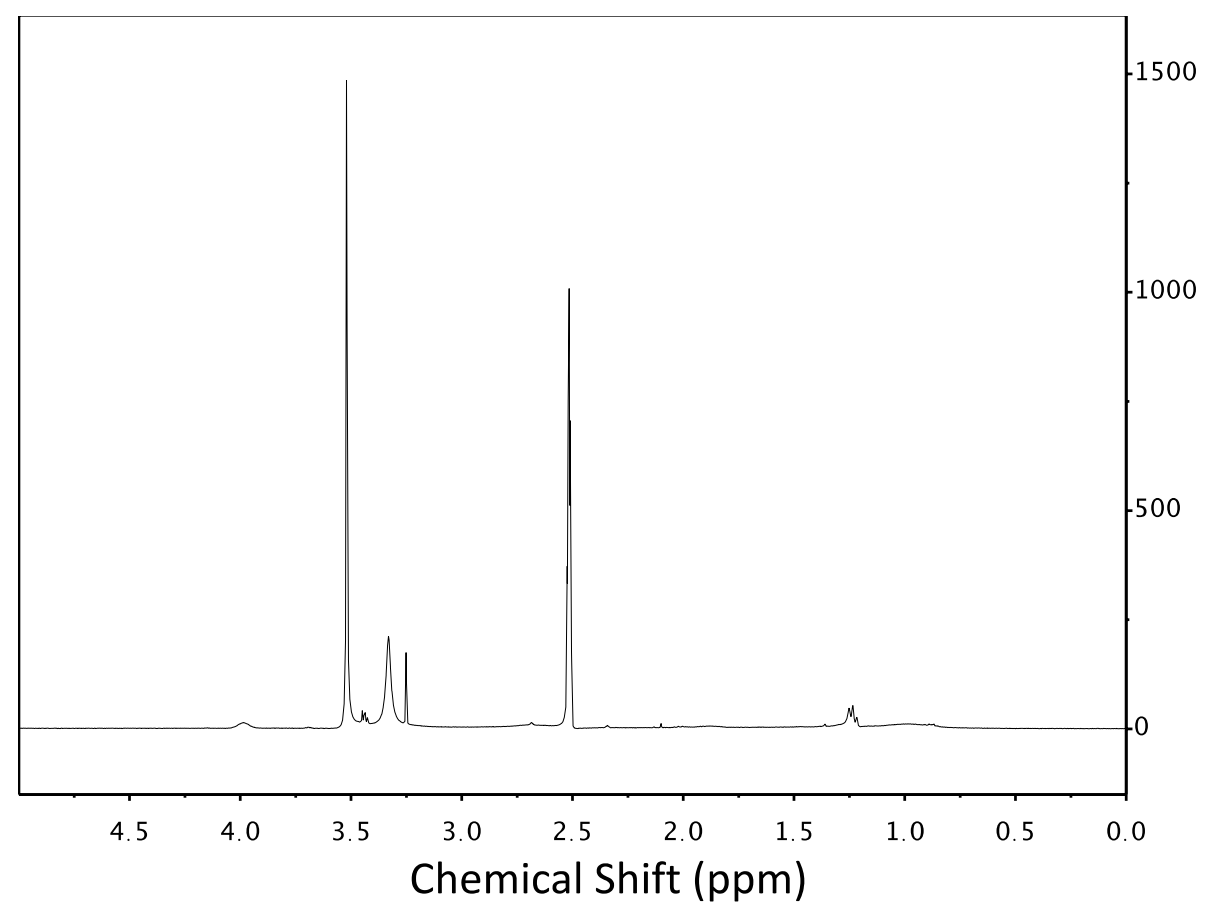

Figure S42. 1H NMR MPAOEt-PIMA-PEG copolymer (50:50) in DMSO-d6.

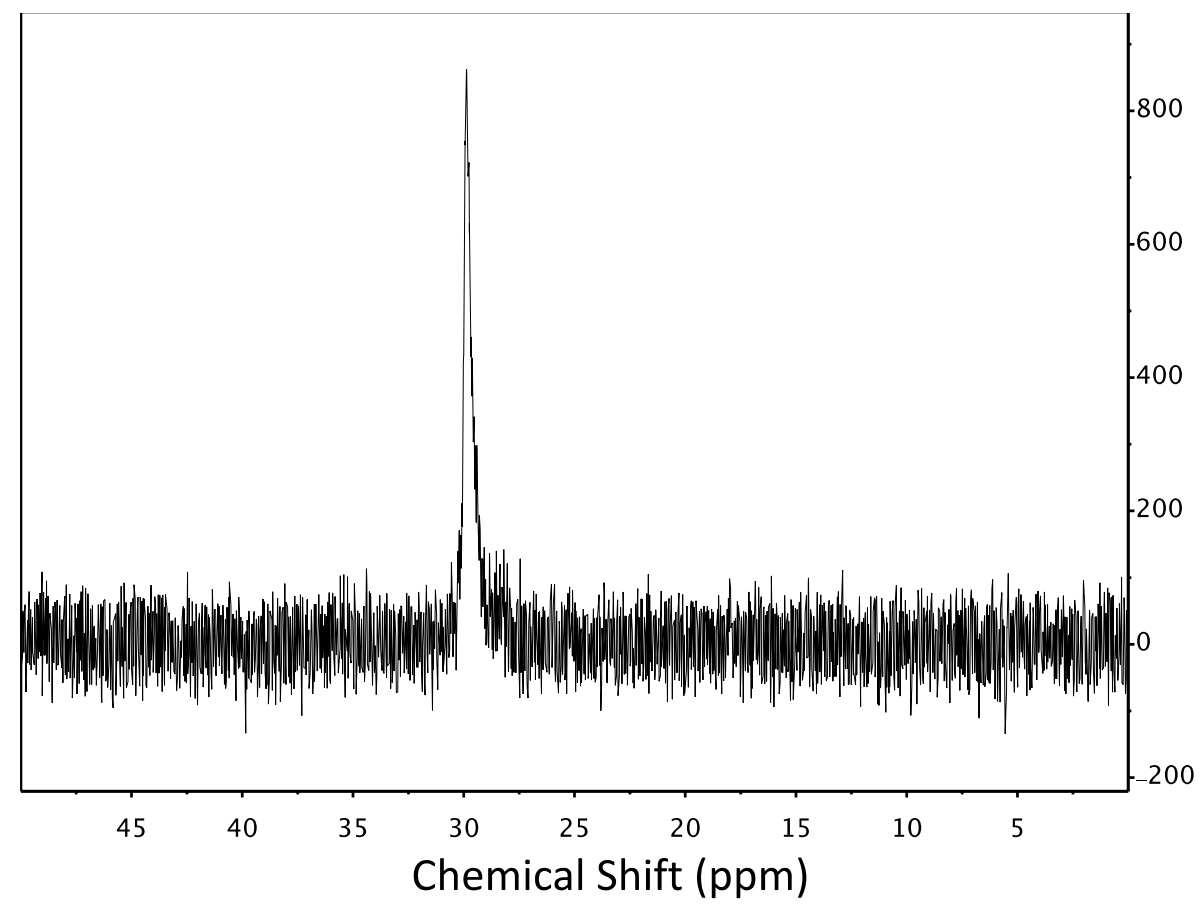

Figure S43. 31P NMR of MPAOEt-PIMA-PEG copolymer (50:50) in DMSO-d6. 


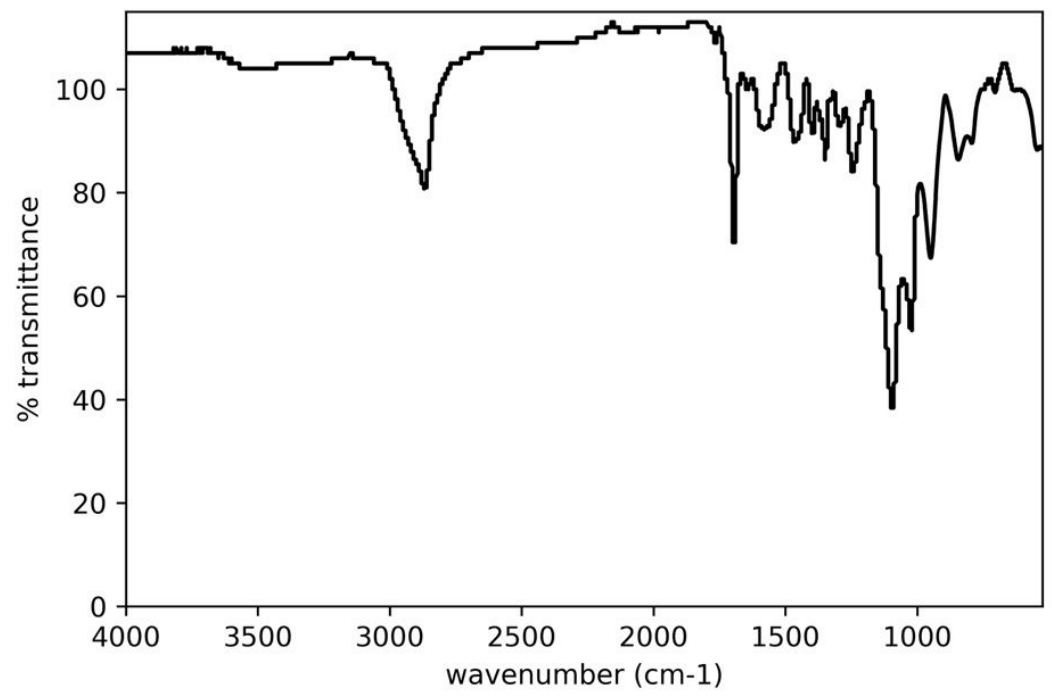

Figure S44. ATR-FTIR data for MPAOEt-PIMA-PEG (50:50) with the following peaks of interest: 3508 and 3295 (O-H stretching, carboxylic acid), 2868 (C-H stretch, alkane), 1695 (C=O stretch, cyclic imide side product), 1577 (C=O stretching; 2० N-H bending), 1466, 1395, 1348, 1292, 1245, 1095 (C-O-C stretch PEG), 1023 (C-O stretch PAOEt \& PEG), 949, $844 \mathrm{~cm}^{-1}$. 
14.9 Data for BPAOEt-PIMA-PEG co-polymers (grafting ratio 25:75)

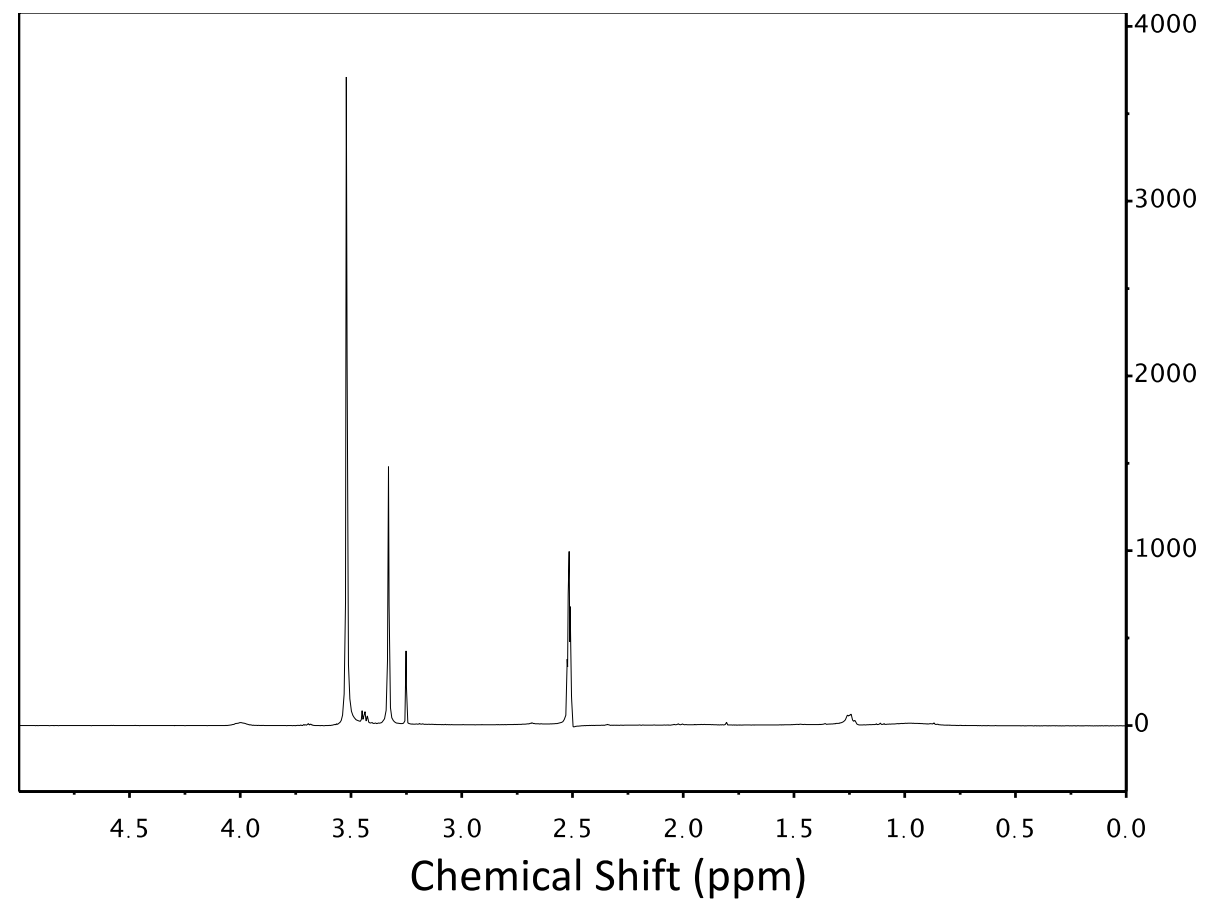

Figure S45. 1H NMR of BPAOEt-PIMA-PEG copolymer (25:75) in DMSO-d6.

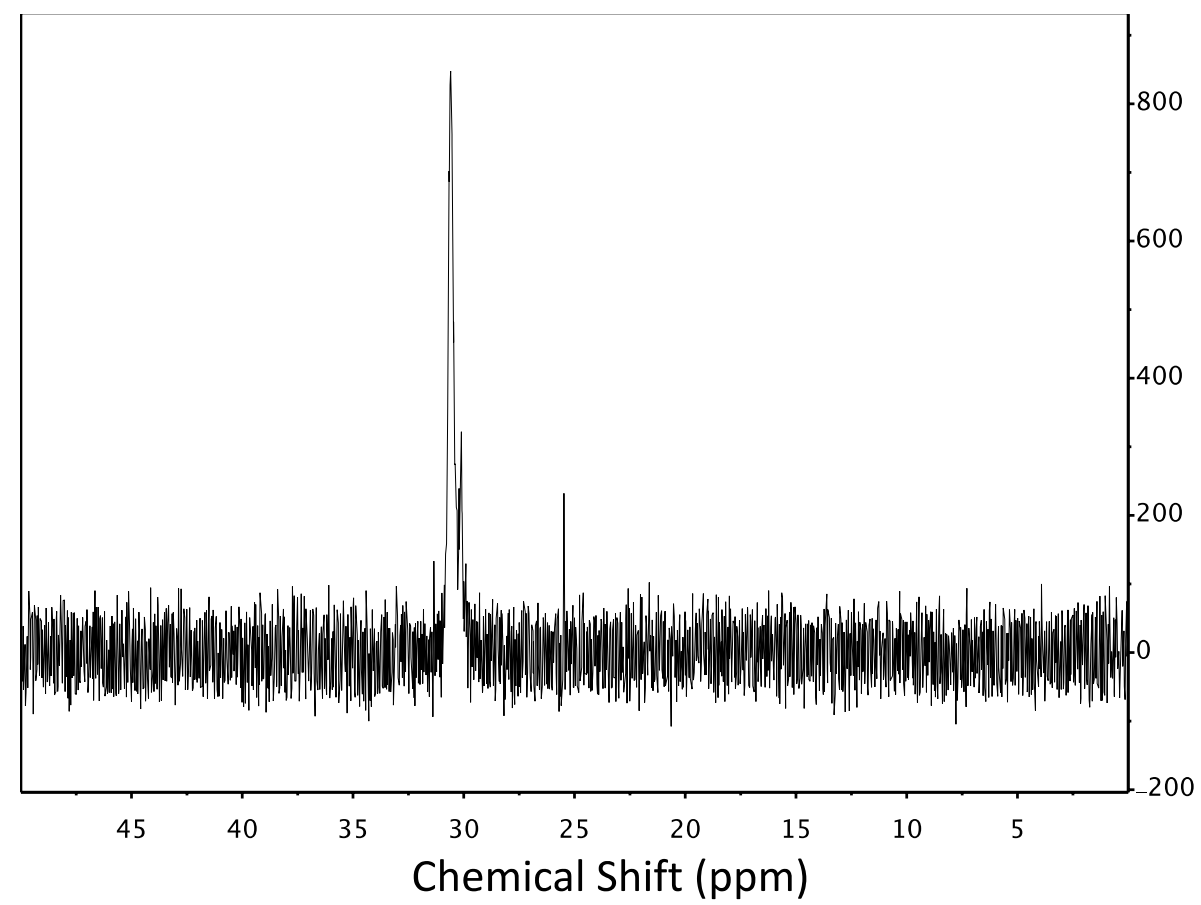

Figure S46. 31P NMR of BPAOEt-PIMA-PEG copolymer (25:75) in DMSO-d6. 


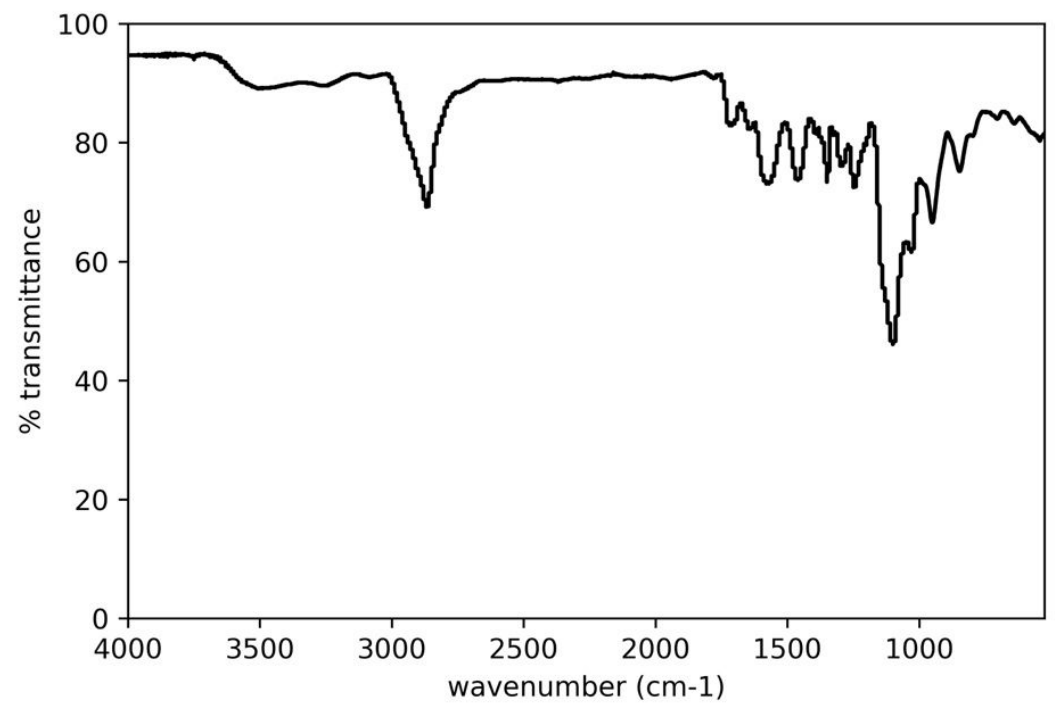

Figure S47. ATR-FTIR data for BPAOEt-PIMA-PEG (25:75) with the following peaks of interest: 3500 and 3300 (O-H stretching, carboxylic acid), 2866 (C-H stretch, alkane), 1716 (C=O stretching, carboxylic acid), 1570 (C=O stretching; 2。 N-H bending), 1458, 1348, 1295, 1246, 1099 (C-O-C stretch PEG), 1030 (C-O stretch PAOEt \& PEG), 950, $847 \mathrm{~cm}^{-1}$. 
14.10 Data for BPAOEt-PIMA-PEG co-polymers (grafting ratio 50:50)

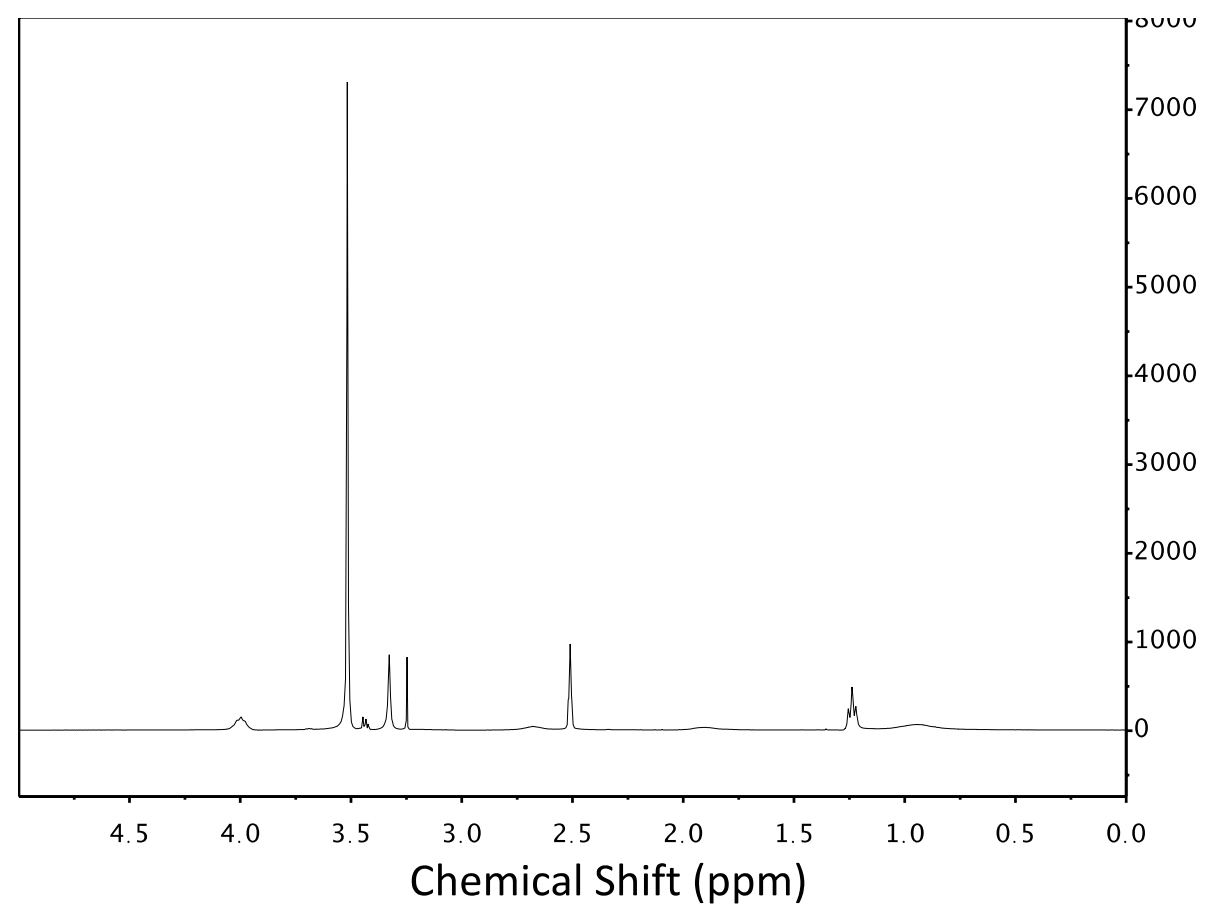

Figure S48. 1H NMR of BPAOEt-PIMA-PEG copolymer (50:50) in DMSO-d6.

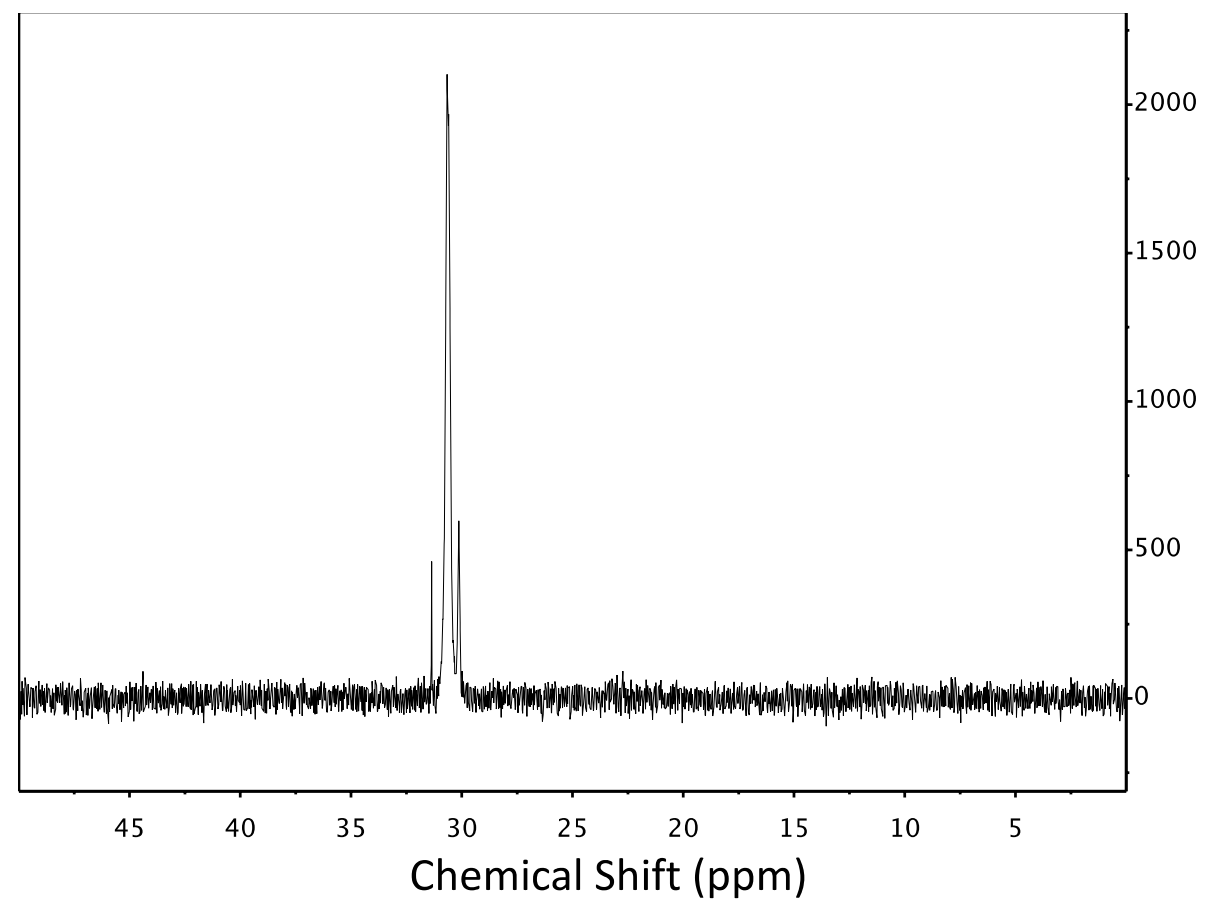

Figure S49. 31P NMR of BPAOEt-PIMA-PEG copolymer (50:50) in DMSO-d6. 


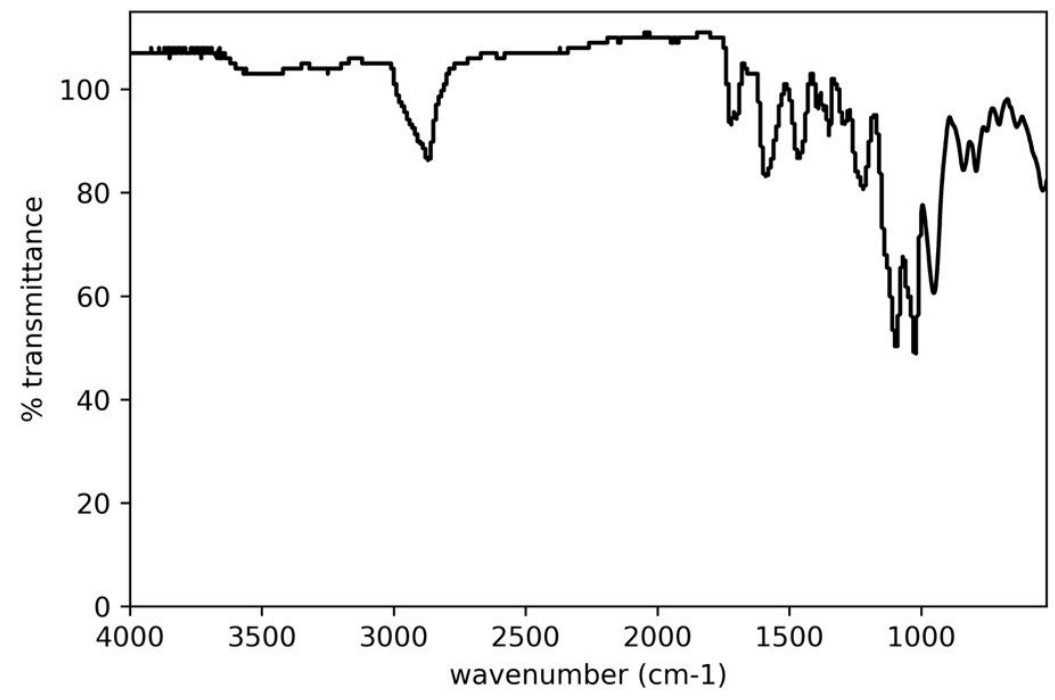

Figure S50. ATR-FTIR data for BPAOEt-PIMA-PEG (50:50) with the following peaks of interest: 3507 and 3241 (O-H stretching, carboxylic acid), 2869 (C-H stretch, alkane), 1720 (C=O stretching, carboxylic acid), 1590 (C=O stretching; $2 \circ \mathrm{N}-\mathrm{H}$ bending), 1458, 1393, 1348, 1292, 1220, 1096 (CO-C stretch PEG), 1024 (C-O stretch PAOEt \& PEG), 952, $840 \mathrm{~cm}^{-1}$. 
14.11 Data for BPAOEt-PIMA-PEG- $\mathrm{N}_{3}$ co-polymers (grafting ratio 50:45:5)

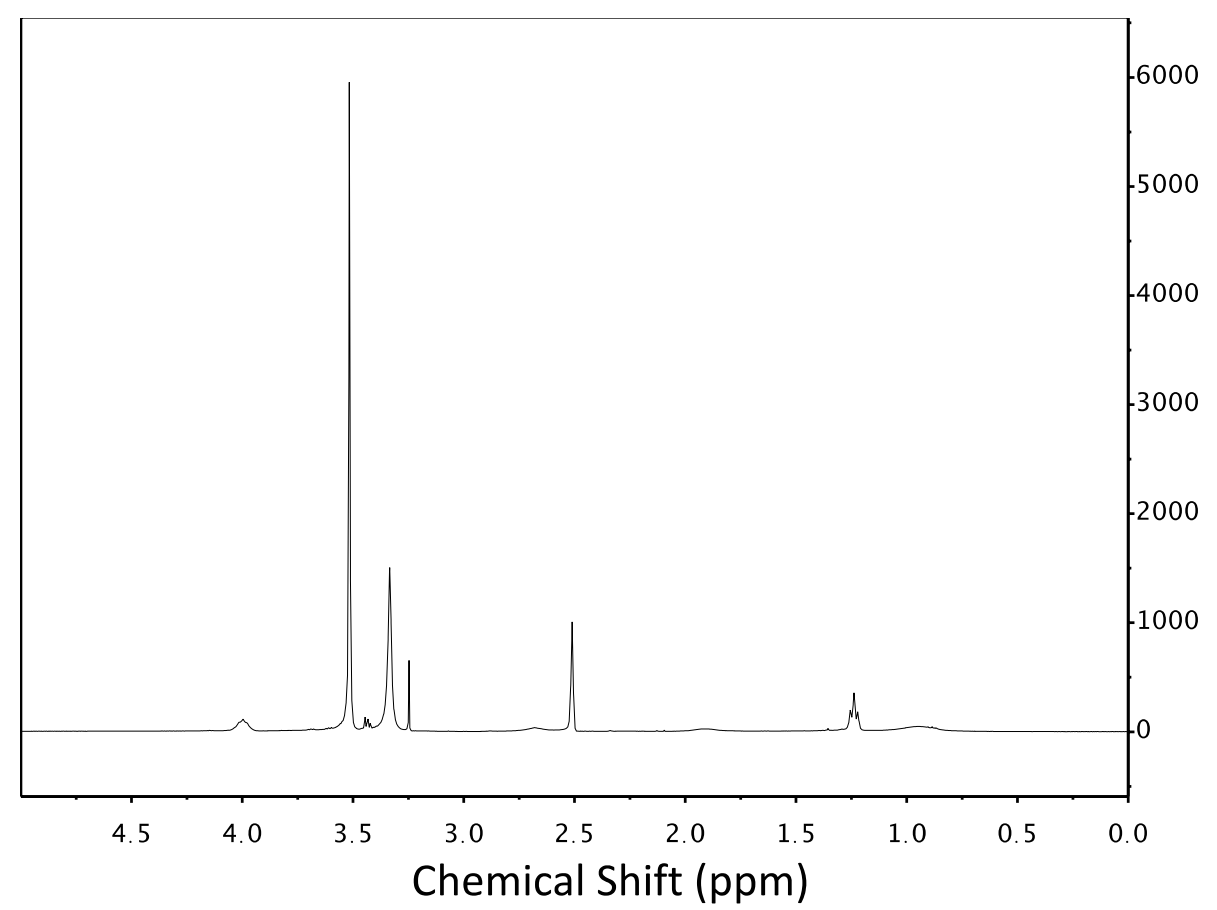

Figure S51. 1H NMR of BPAOEt-PIMA-PEG-N ${ }_{3}$ copolymer (50:45:5) in DMSO-d6.

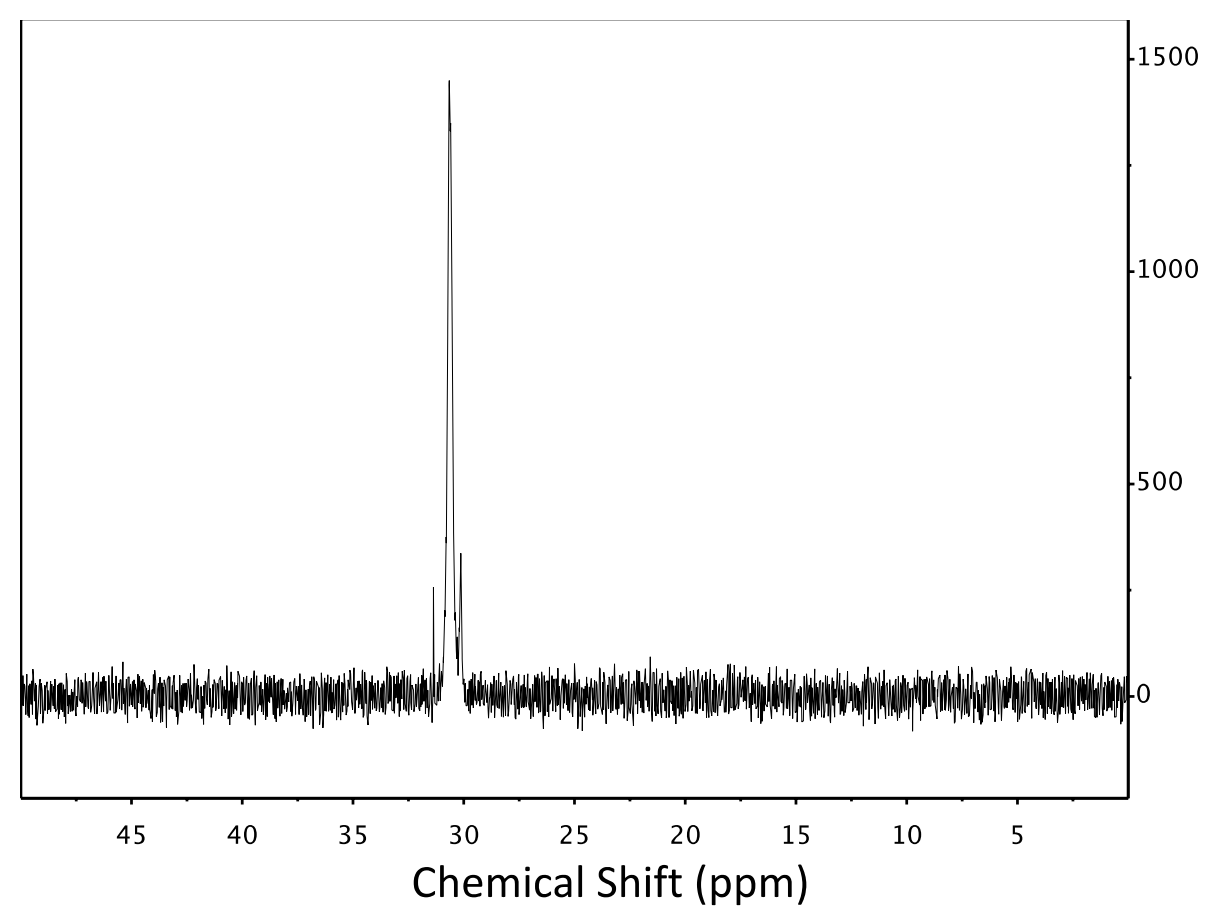

Figure S52. 31P NMR of BPAOEt-PIMA-PEG- $N_{3}$ copolymer (50:45:5) in DMSO-d6. 


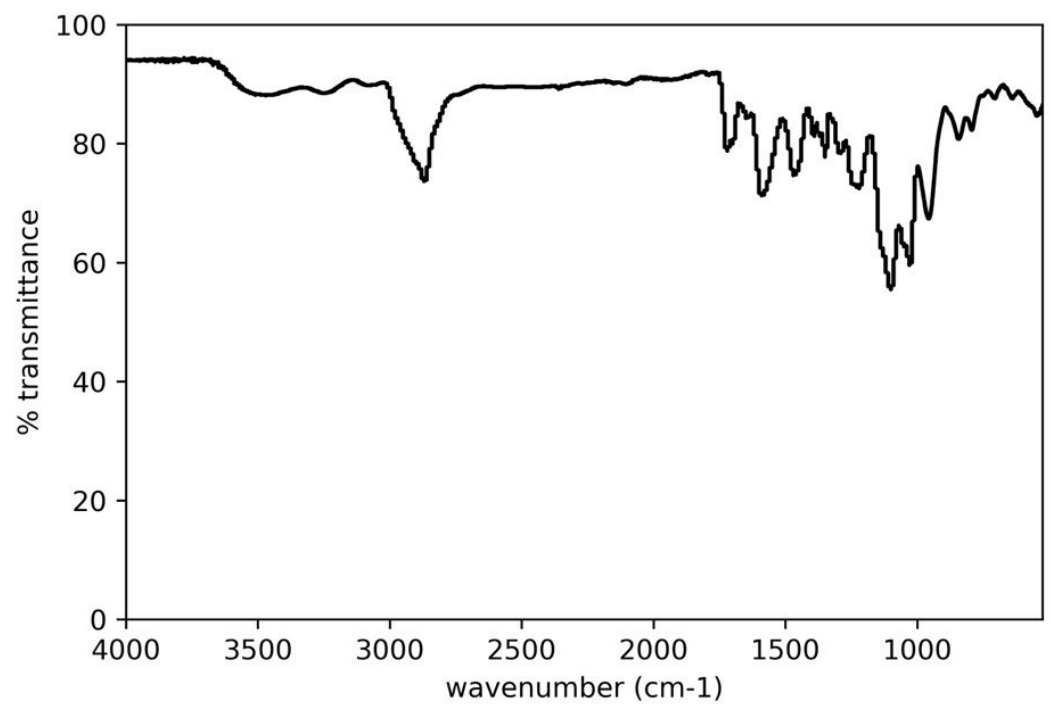

Figure S53. ATR-FTIR data for BPAOEt-PIMA-PEG-N $\mathrm{N}_{3}$ (50:45:5) with the following peaks of interest: 3472 and 3230 (O-H stretching, carboxylic acid), 2868 (C-H stretch, alkanes), 2103 ( $\mathrm{N}_{3}$ stretch), 1721 ( $\mathrm{C}=\mathrm{O}$ stretch, carboxylic acid), 1589 (C=O stretching; 2० $\mathrm{N}-\mathrm{H}$ bending), 1470, 1393, 1348, 1221, 1100 (C-O-C stretch PEG), 1028 (C-O stretch PAOEt \& PEG), 957, $843 \mathrm{~cm}^{-1}$. 
14.12 Data for MPAOH-PIMA-PEG co-polymers (grafting ratio 25:75)

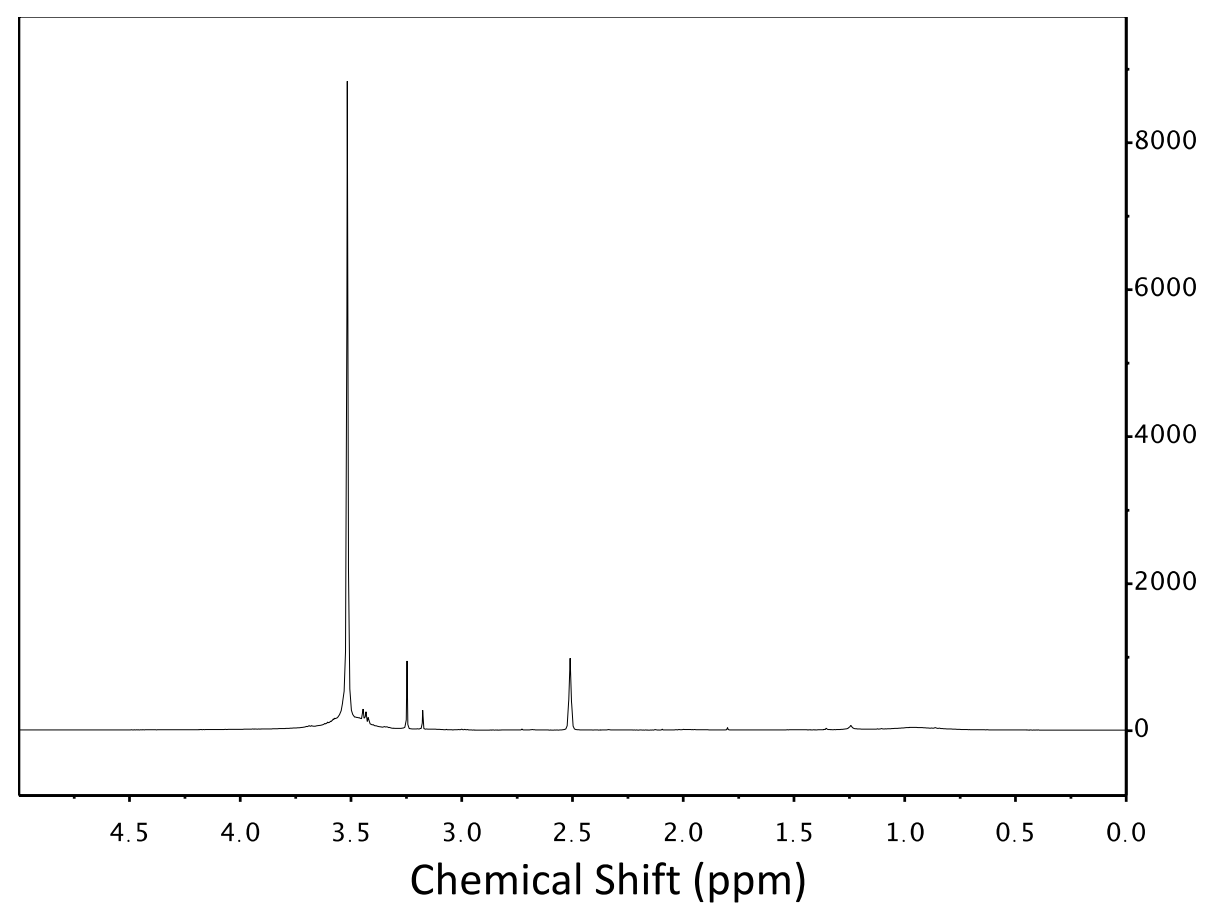

Figure S54. 1H NMR of MPAOH-PIMA-PEG copolymer (25:75) in DMSO-d6.

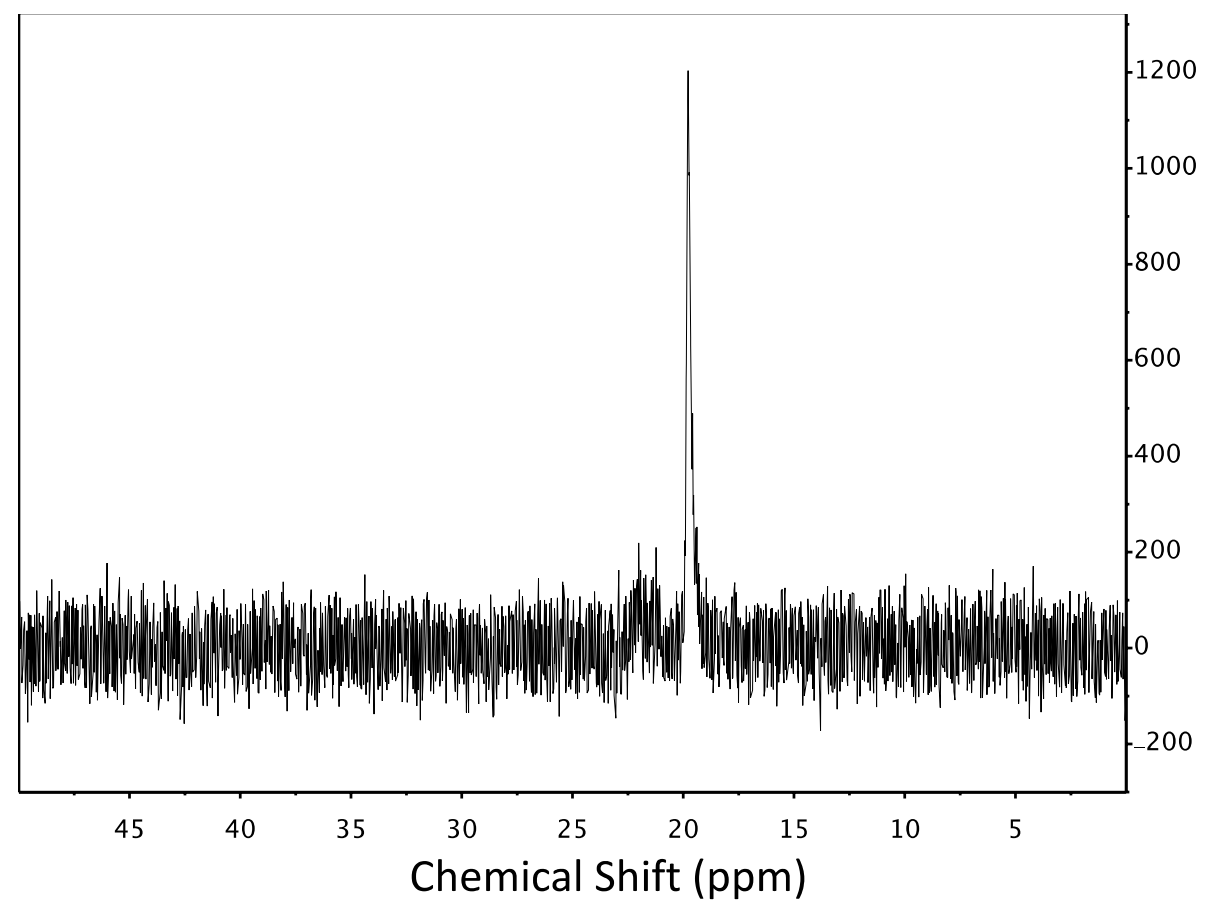

Figure S55. 31P NMR of MPAOH-PIMA-PEG copolymer (25:75) in DMSO-d6. 


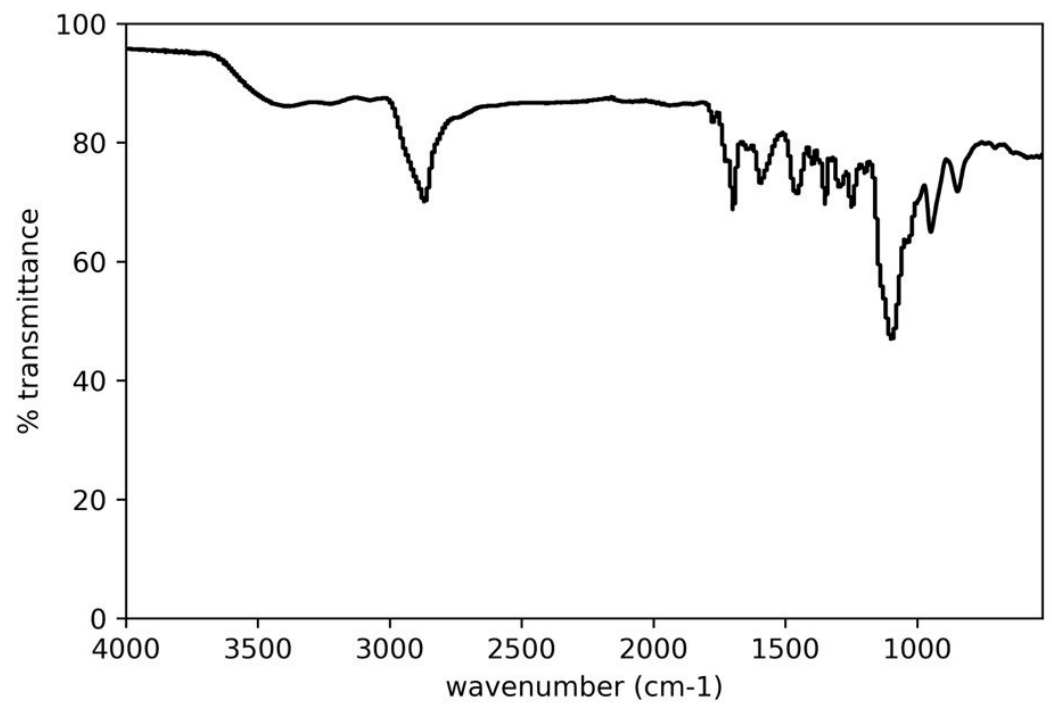

Figure S56. ATR-FTIR data for MPAOH-PIMA-PEG (25:75) with the following peaks of interest: 3400 and 3220(O-H stretching, carboxylic acid), 2868 (C-H stretch, alkanes), 1698 (C=O stretch, cyclic imide side product), 1594 (C=O stretching; $2 \circ \mathrm{N}-\mathrm{H}$ bending), 1455, 1397, 1348, 1296, 1248, 1097 (C-O-C stretch PEG), 1038 (C-O stretch PAOEt \& PEG), 949, $847 \mathrm{~cm}^{-1}$. 
14.13 Data for MPAOH-PIMA-PEG co-polymers (grafting ratio 50:50)

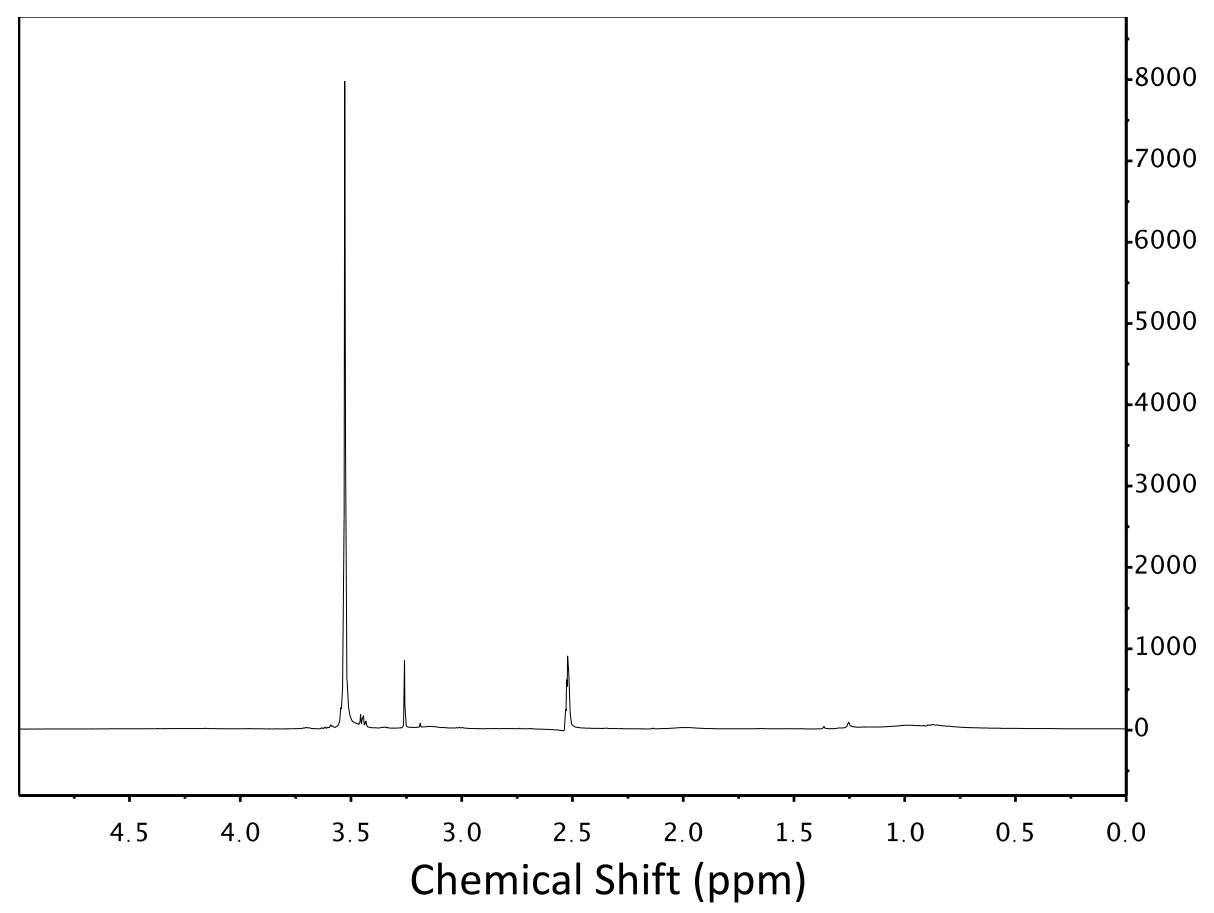

Figure S57. 1H NMR MPAOH-PIMA-PEG copolymer (50:50) in DMSO-d6.

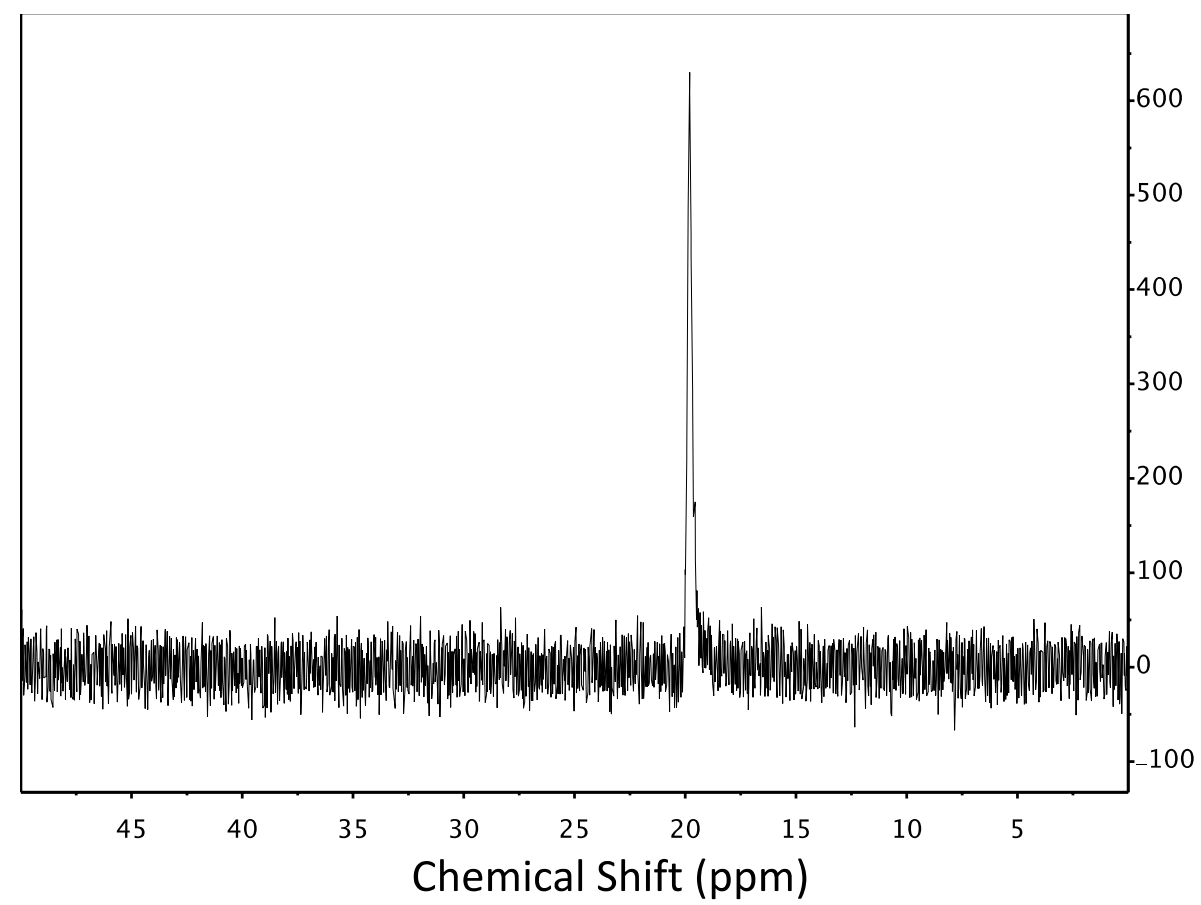

Figure S58. 31P NMR of MPAOH-PIMA-PEG copolymer (50:50) in DMSO-d6. 


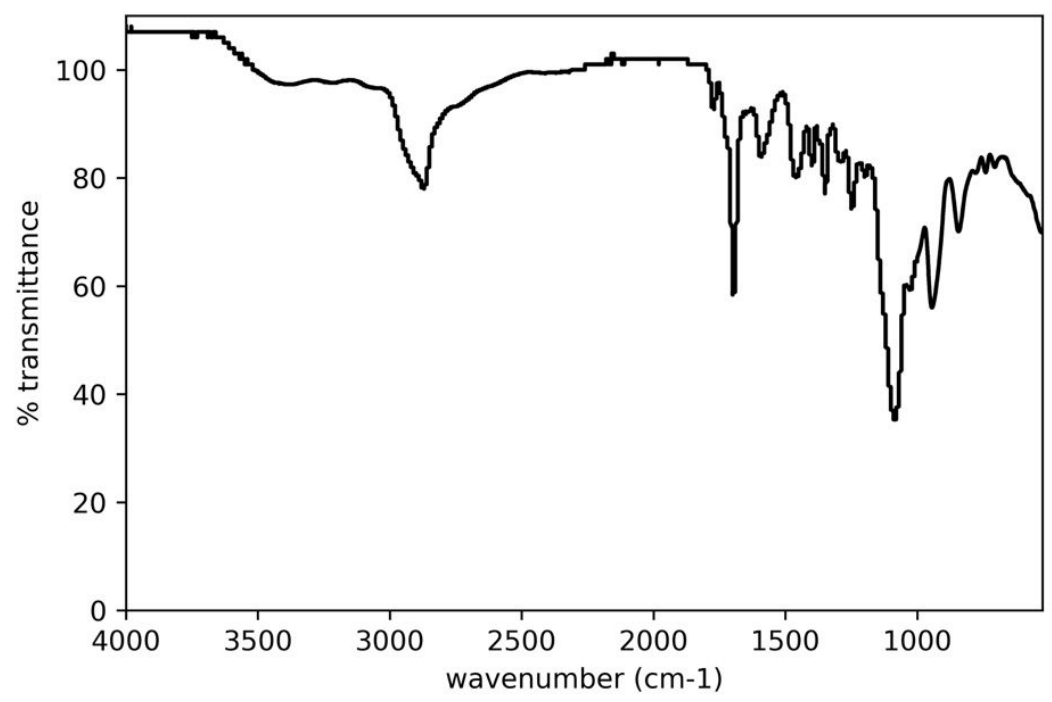

Figure S59. ATR-FTIR data for MPAOH-PIMA-PEG (50:50) with the following peaks of interest: 3421 and 3232(O-H stretching, carboxylic acid), 2872 (C-H stretch, alkanes), 1696 (C=O stretch, cyclic imide side product), 1593 (C=O stretching; $2 \circ \mathrm{N}-\mathrm{H}$ bending), 1457, 1399, 1348, 1289, 1248, 1085 (C-O-C stretch PEG), 1027 (C-O stretch PAOEt \& PEG), 944, $843 \mathrm{~cm}^{-1}$. 
14.14 Data for BPAOH-PIMA-PEG co-polymers (grafting ratio 25:75)

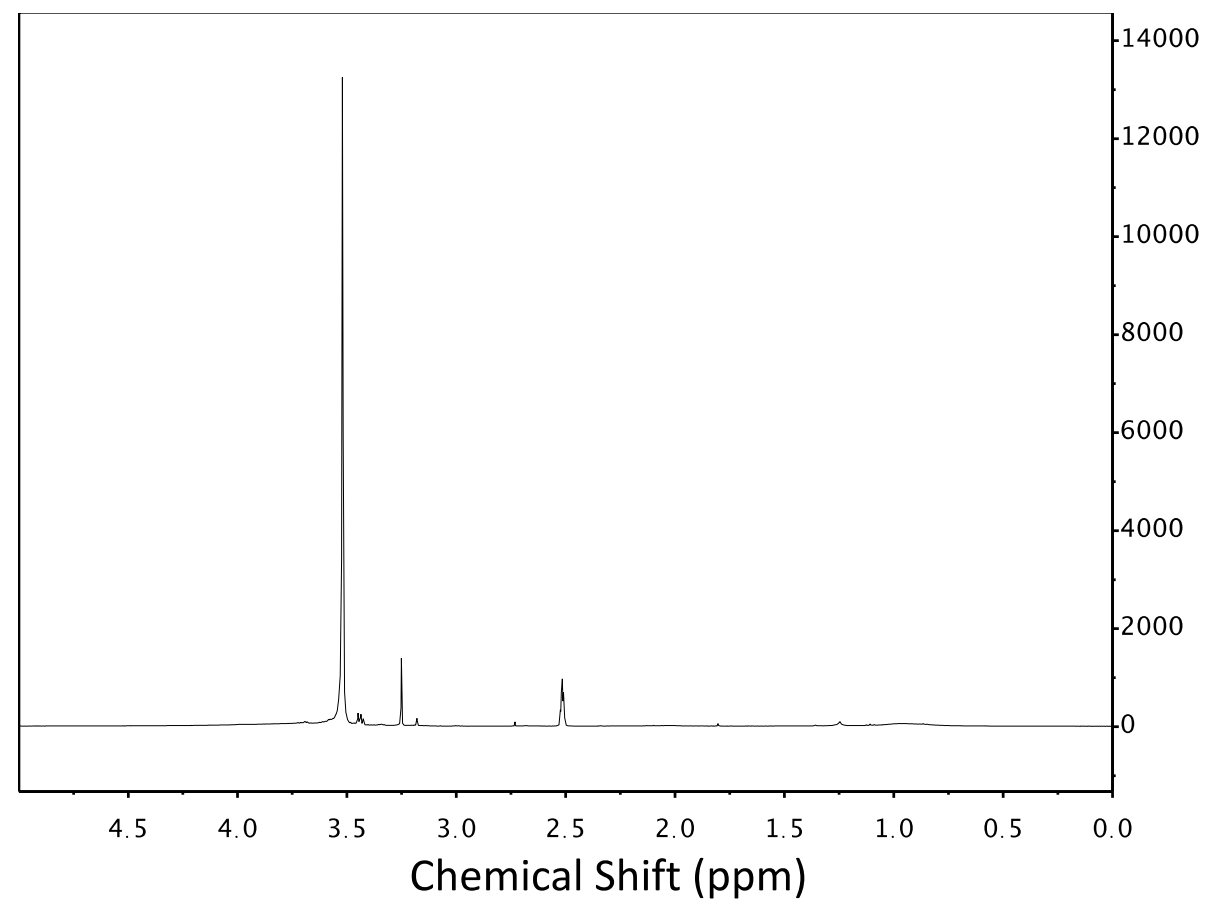

Figure S60. 1H NMR of BPAOH-PIMA-PEG copolymer (25:75) in DMSO-d6.

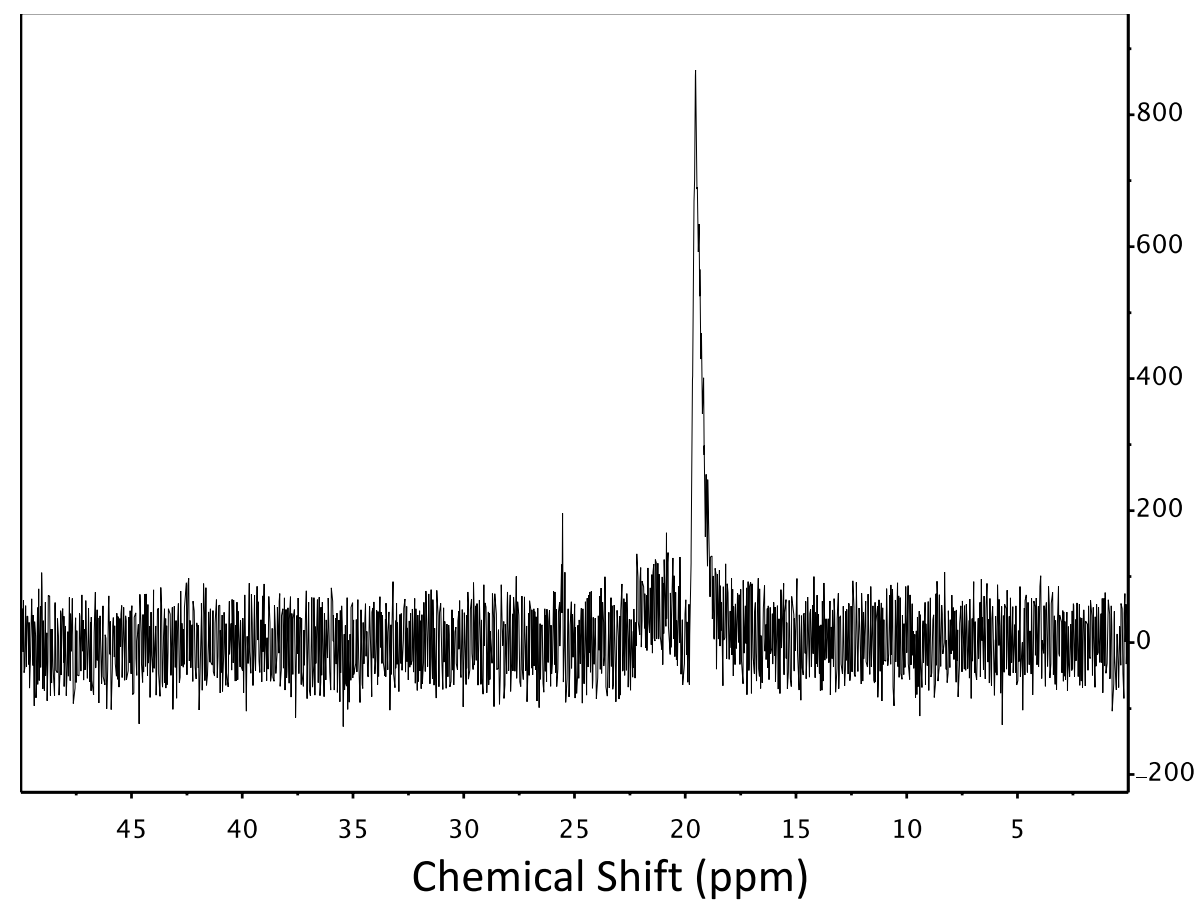

Figure S61. 31P NMR of BPAOH-PIMA-PEG copolymer (25:75) in DMSO-d6. 


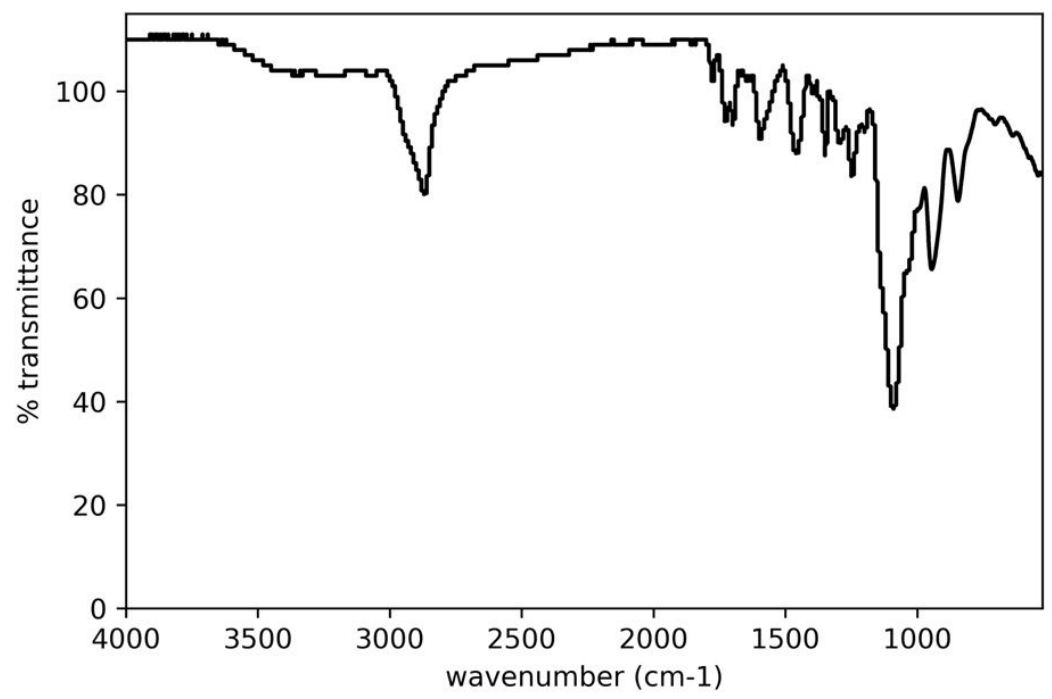

Figure S62. ATR-FTIR data for BPAOH-PIMA-PEG (25:75) with the following peaks of interest: 3419 (O-H stretching, carboxylic acid), 2868 (C-H stretch, alkanes), 1727 (C=O stretch, carboxylic acid), 1698 ( $C=$ O stretch, cyclic imide side product), 1595 ( $C=$ O stretching; $2 \circ \mathrm{N}-\mathrm{H}$ bending), 1456, 1348, 1294, 1247, 1091 (C-O-C stretch PEG), 945, $845 \mathrm{~cm}^{-1}$. 
14.15 Data for BPAOH-PIMA-PEG co-polymers (grafting ratio 50:50)

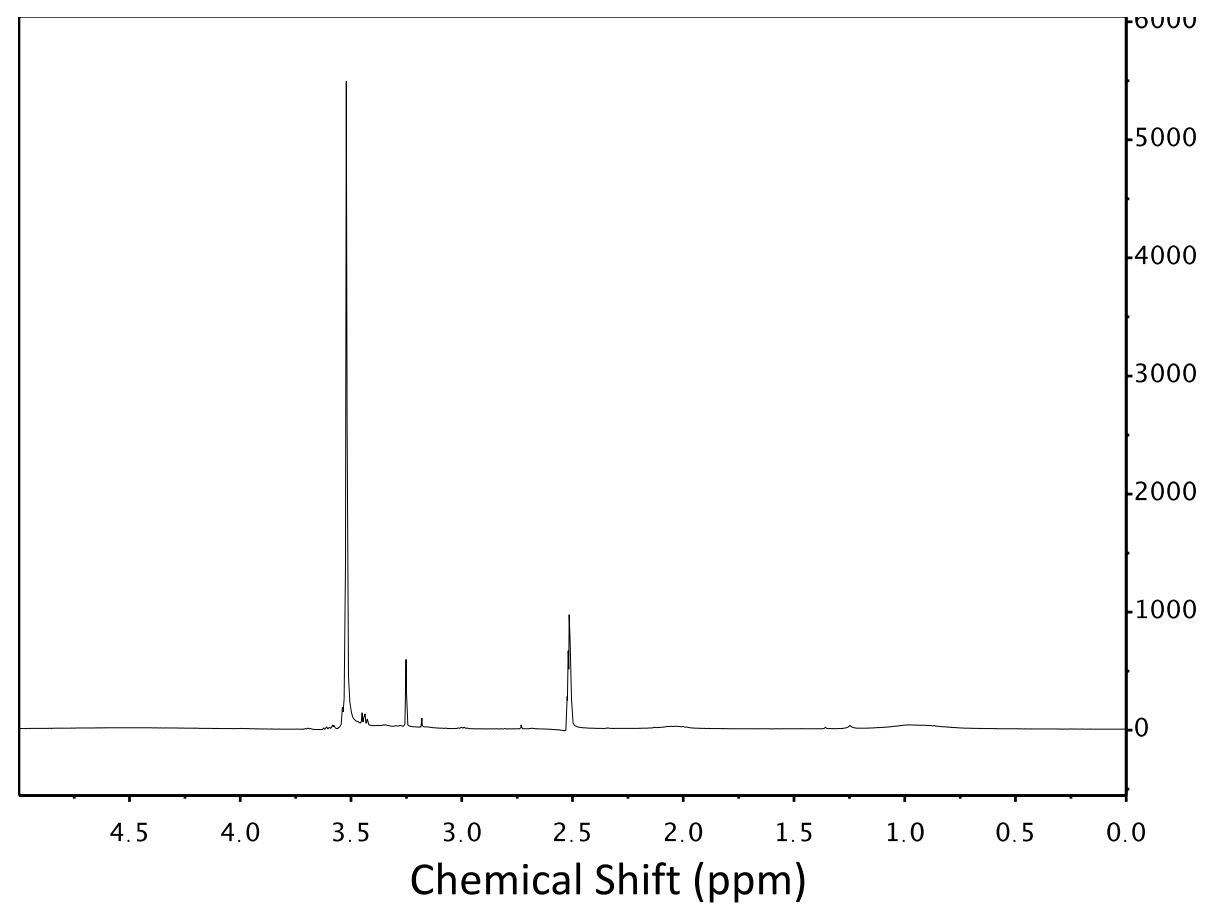

Figure S63. 1H NMR of BPAOH-PIMA-PEG copolymer (50:50) in DMSO-d6.

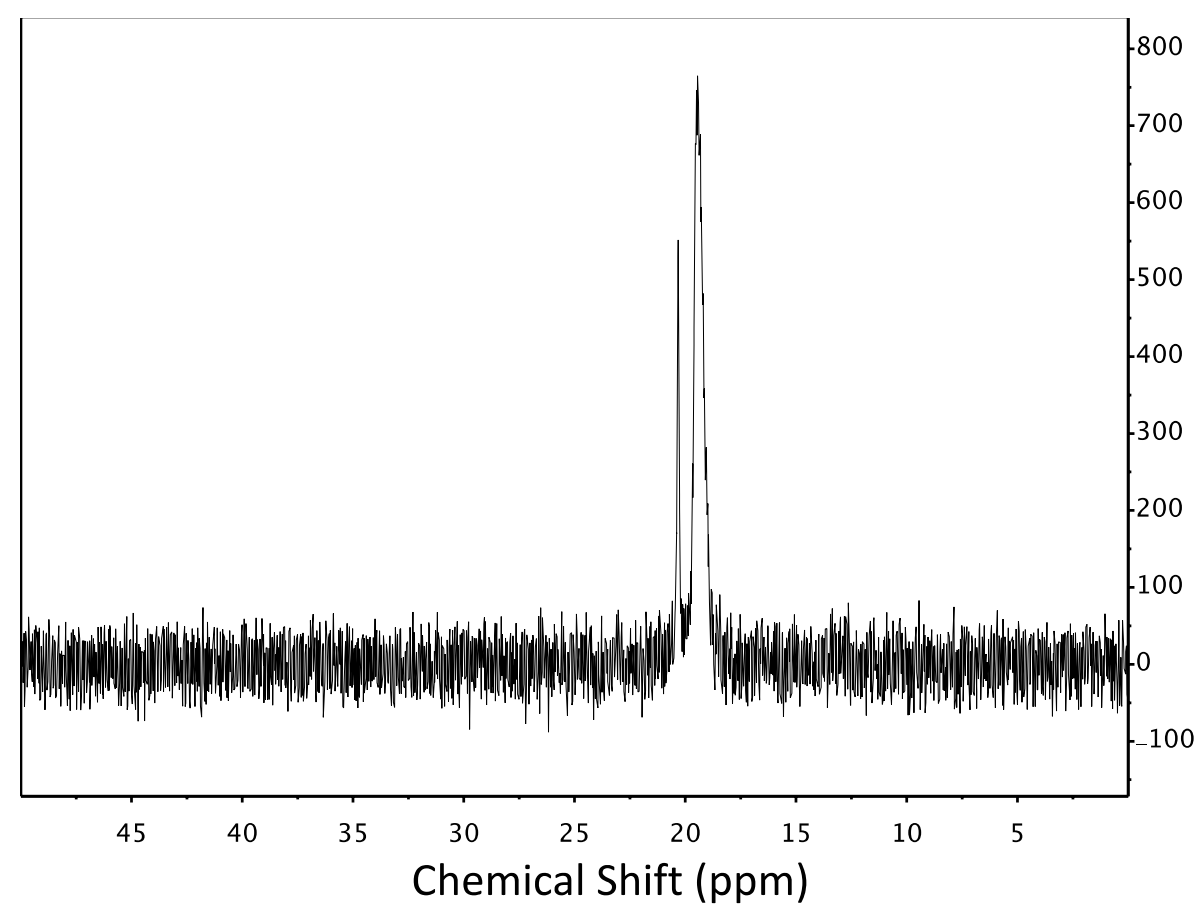

Figure S64. 31P NMR of BPAOH-PIMA-PEG copolymer (50:50) in DMSO-d6. 


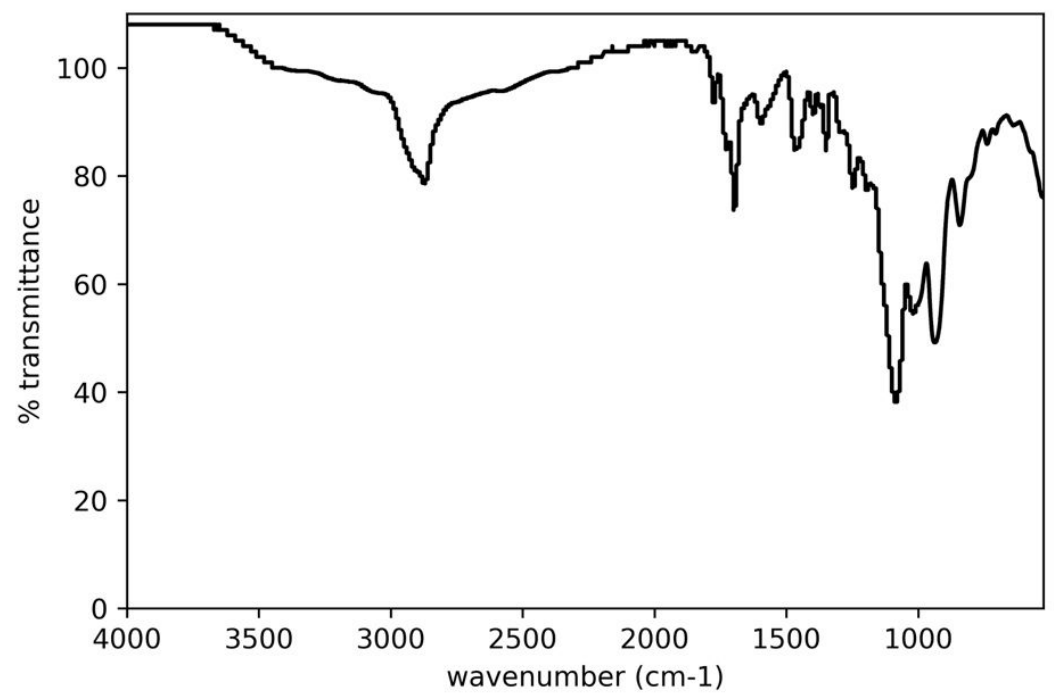

Figure S65. ATR-FTIR data for BPAOH-PIMA-PEG (50:50) with the following peaks of interest: 3420 and 3230 (O-H stretching, carboxylic acid), 2872 (C-H stretch, alkanes), 1697 (C=O stretch, cyclic imide side product), 1596 (C=O stretching; $2 \circ \mathrm{N}-\mathrm{H}$ bending), 1466, 1397, 1349, 1247, 1085 (C-OC stretch PEG), 1019 (C-O stretch PAOEt \& PEG), 938, $843 \mathrm{~cm}^{-1}$. 
14.16 Data for BPAOH-PIMA-PEG- $\mathrm{N}_{3}$ co-polymers (grafting ratio 50:45:5)

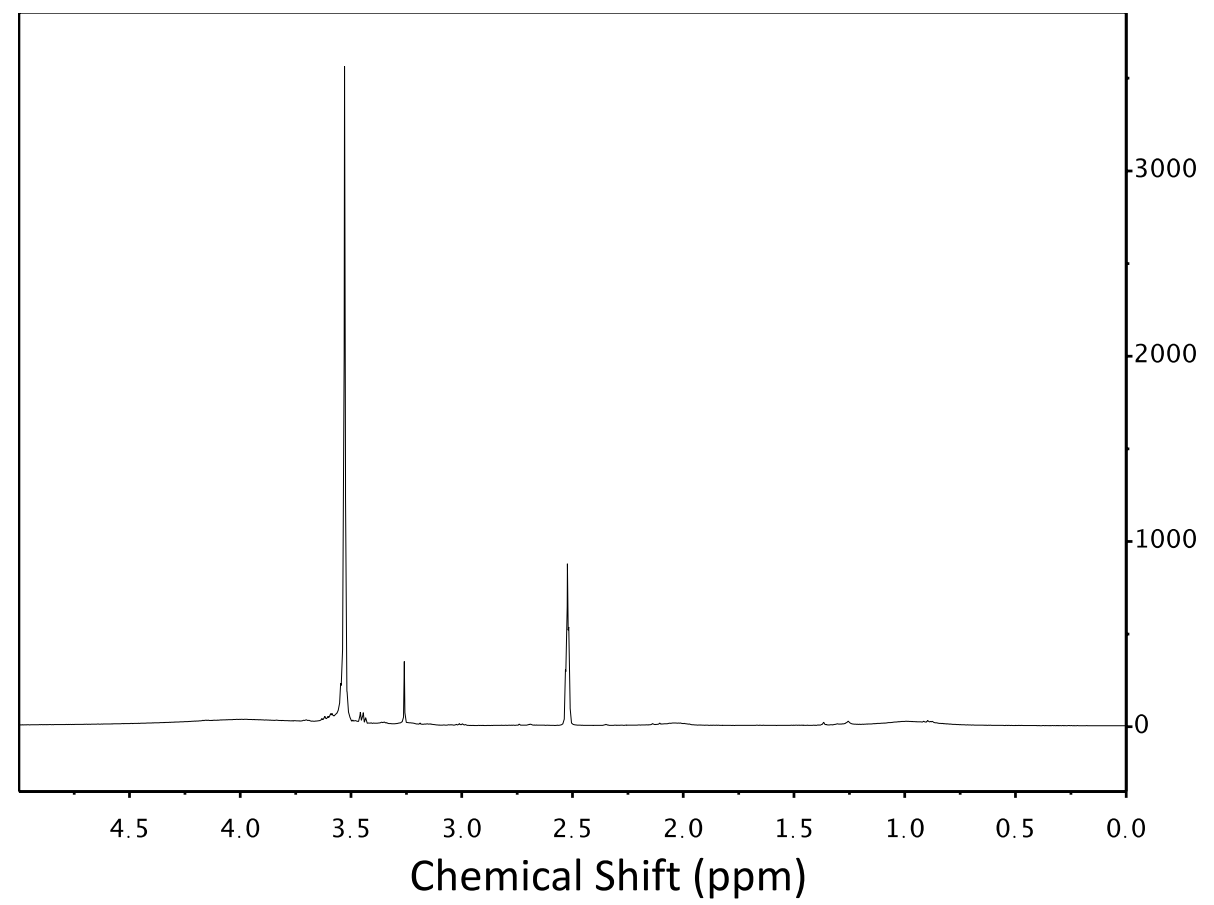

Figure S66. 1H NMR of BPAOH-PIMA-PEG-N ${ }_{3}$ copolymer (50:45:5) in DMSO-d6.

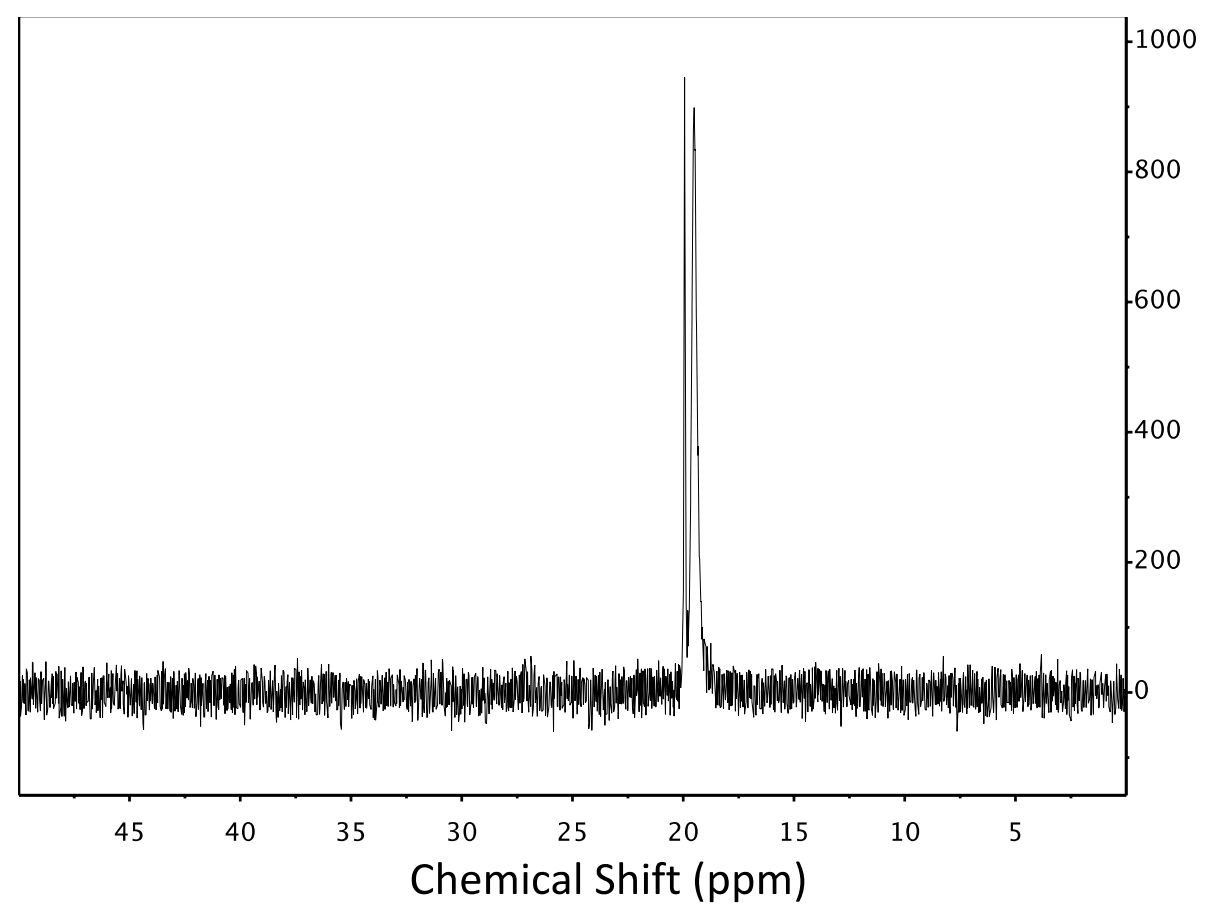

Figure S67. 31P NMR of BPAOH-PIMA-PEG- $\mathrm{N}_{3}$ copolymer (50:45:5) in DMSO-d6. 


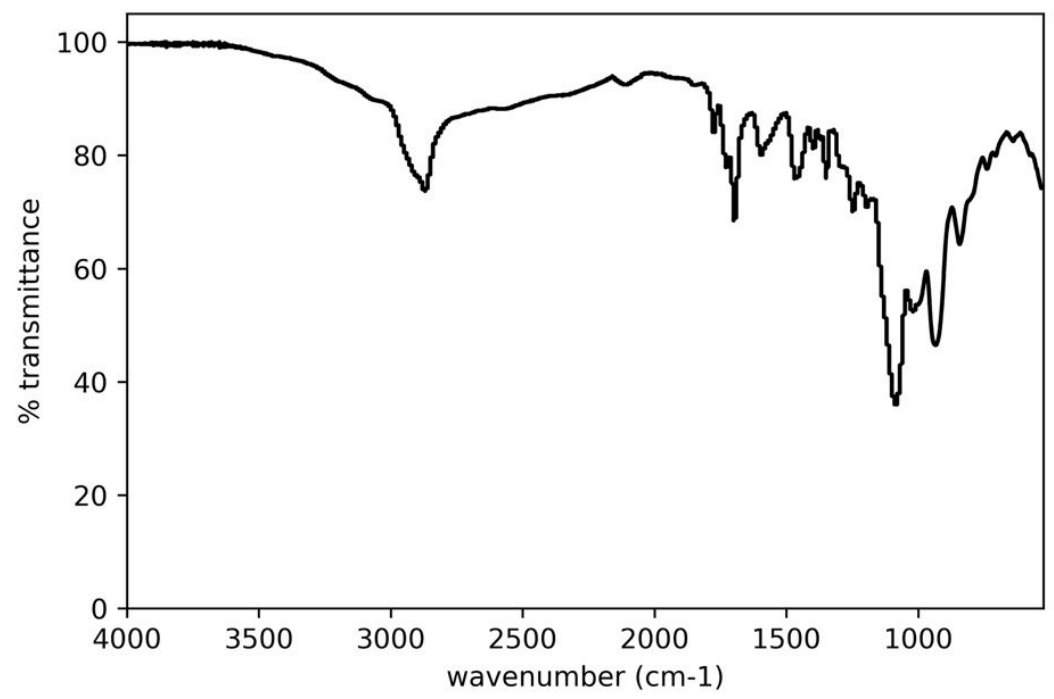

Figure S68. ATR-FTIR data for BPAOH-PIMA-PEG-N $\mathrm{N}_{3}$ (50:45:5) with the following peaks of interest:

3478 (O-H stretching, carboxylic acid), 2870 (C-H stretch, alkanes), 2112 ( $\mathrm{N}_{3}$ stretch), 1697 (C=O stretch, cyclic imide side product), 1596 (C=O stretching; 2॰ N-H bending), 1466, 1397, 1349, 1248, 1085 (C-O-C stretch PEG), 1020 (C-O stretch PAOEt \& PEG), 934, $843 \mathrm{~cm}^{-1}$. 
14.17 Summary of Characterization Data for Protected Polymers:

MPAOEt-PIMA-PEG (grafting ratio of 25:75):

31P NMR (162 MHz, DMSO-d6) $\delta$ 29.84. ATR-FTIR: 3500 and 3286 (O-H stretching, carboxylic acid), 2867 ( $\mathrm{C}-\mathrm{H}$ stretching, alkane), 1697 ( $\mathrm{C}=\mathrm{O}$ stretch, cyclic imide side product), 1575 ( $\mathrm{C=O}$ stretching; 2० $\mathrm{N}-\mathrm{H}$ bending), 1456, 1394, 1296, 1247, 1091 (C-O-C stretch PEG), 1028 (C-O stretch PAOEt \& PEG), 947, $846 \mathrm{~cm}^{-1}$.

MPAOEt-PIMA-PEG (grafting ratio of 50:50):

31P NMR (162 MHz, DMSO-d6) $\delta$ 29.85. ATR-FTIR: 3508 and 3295 (O-H stretching, carboxylic acid), 2868 ( $\mathrm{C}-\mathrm{H}$ stretch, alkane), 1695 ( $\mathrm{C}=\mathrm{O}$ stretch, cyclic imide side product), 1577 ( $\mathrm{C=0}$ stretching; 2. N-H bending), 1466, 1395, 1348, 1292, 1245, 1095 (C-O-C stretch PEG), 1023 (C-O stretch PAOEt \& PEG), $949,844 \mathrm{~cm}^{-1}$.

\section{BPAOEt-PIMA-PEG (grafting ratio of 25:75):}

31P NMR (162 MHz, DMSO-d6) 8 30.35. ATR-FTIR: 3500 and 3270 (O-H stretching, carboxylic acid), 2866 (C-H stretch, alkane), 1716 ( $\mathrm{C}=\mathrm{O}$ stretching, carboxylic acid), 1570 ( $\mathrm{C}=\mathrm{O}$ stretching; $2 \circ \mathrm{N}-\mathrm{H}$ bending), 1458, 1348, 1295, 1246, 1099 (C-O-C stretch PEG), 1030 (C-O stretch PAOEt \& PEG), $950,847 \mathrm{~cm}^{-1}$.

BPAOEt-PIMA-PEG (grafting ratio of 50:50):

31P NMR (162 MHz, DMSO-d6) $\delta$ 30.63. ATR-FTIR: 3507 and 3241 (O-H stretching, carboxylic acid), 2869 (C-H stretch, alkane), 1720 ( $\mathrm{C}=\mathrm{O}$ stretching, carboxylic acid), 1590 ( $\mathrm{C=}=\mathrm{O}$ stretching; 2。 N-H bending), 1458, 1393, 1348, 1292, 1220, 1096 (C-O-C stretch PEG), 1024 (C-O stretch PAOEt \& PEG), $952,840 \mathrm{~cm}^{-1}$. 
BPAOEt-PIMA-PEG-N3 (grafting ratio of 50:45:5):

31P NMR (162 MHz, DMSO-d6) $\delta$ 30.36. ATR-FTIR: 3472 and 3230 (O-H stretching, carboxylic acid), 2868 (C-H stretch, alkanes), 2103 ( $\mathrm{N}_{3}$ stretch), 1721 (C=O stretch, carboxylic acid), 1589 (C=O stretching; $2 \circ \mathrm{N}-\mathrm{H}$ bending), 1470, 1393, 1348, 1221, 1100 (C-O-C stretch PEG), 1028 (C-O stretch PAOEt \& PEG), 957, $843 \mathrm{~cm}^{-1}$. 


\subsection{Summary of Characterization Data for Final Polymers:}

\section{MPAOH-PIMA-PEG (grafting ratio of 25:75):}

31P NMR (162 MHz, DMSO-d6) $\delta$ 19.77. ATR-FTIR: 3400 and 3220(O-H stretching, carboxylic acid), 2868 (C-H stretch, alkanes), 1698 (C=O stretch, cyclic imide side product), 1594 (C=O stretching; 2० N-H bending), 1455, 1397, 1348, 1296, 1248, 1097 (C-O-C stretch PEG), 1038 (C-O stretch PAOEt \& PEG), 949, $847 \mathrm{~cm}^{-1}$.

MPAOH-PIMA-PEG (grafting ratio of 50:50):

31P NMR (162 MHz, DMSO-d6) $\delta$ 19.80. ATR-FTIR: 3421 and 3232(O-H stretching, carboxylic acid), 2872 (C-H stretch, alkanes), 1696 (C=O stretch, cyclic imide side product), 1593 (C=O stretching; 2० N-H bending), 1457, 1399, 1348, 1289, 1248, 1085 (C-O-C stretch PEG), 1027 (C-O stretch PAOEt \& PEG), $944,843 \mathrm{~cm}^{-1}$.

\section{BPAOH-PIMA-PEG (grafting ratio of 25:75):}

31P NMR (162 MHz, DMSO-d6) $\delta$ 19.45. ATR-FTIR: 3419 (O-H stretching, carboxylic acid), 2868 (CH stretch, alkanes), 1727 ( $\mathrm{C}=\mathrm{O}$ stretch, carboxylic acid), 1698 ( $\mathrm{C}=\mathrm{O}$ stretch, cyclic imide side product), 1595 (C=O stretching; 2॰ N-H bending), 1456, 1348, 1294, 1247, 1091 (C-O-C stretch PEG), 945, $845 \mathrm{~cm}^{-1}$.

BPAOH-PIMA-PEG (grafting ratio of 50:50):

31P NMR (162 MHz, DMSO-d6) $\delta$ 19.86. ATR-FTIR: 3420 and 3230 (O-H stretching, carboxylic acid), 2872 (C-H stretch, alkanes), 1697 (C=O stretch, cyclic imide side product), 1596 (C=O stretching; 2० N-H bending), 1466, 1397, 1349, 1247, 1085 (C-O-C stretch PEG), 1019 (C-O stretch PAOEt \& PEG), 938, $843 \mathrm{~cm}^{-1}$.

BPAOH-PIMA-PEG-N3 (grafting ratio of 50:45:5): 
31P NMR (162 MHz, DMSO-d6) $\delta$ 19.72. ATR-FTIR: 3478 (O-H stretching, carboxylic acid), 2870 (CH stretch, alkanes), 2112 ( $\mathrm{N}_{3}$ stretch), 1697 ( $\mathrm{C}=\mathrm{O}$ stretch, cyclic imide side product), 1596 ( $\mathrm{C}=0$ stretching; $2 \circ \mathrm{N}-\mathrm{H}$ bending), 1466, 1397, 1349, 1248, 1085 (C-O-C stretch PEG), 1020 (C-O stretch PAOEt \& PEG), 934, $843 \mathrm{~cm}^{-1}$. 


\section{References}

1. Uddayasankar, U; Shergill, R. T.; Krull, U. J.; Evaluation of Nanoparticle-Ligand Distributions to Determine Nanoparticle Concentration. Anal. Chem., 2015, 87, 1297 - 1305.

2. Mei, B. C., Susumu, K., Medintz, I. L., Delehanty, J. B., Mountziaris, T. J., Mattoussi, H.; Modular poly(ethylene glycol) ligands for biocompatible semiconductor and gold nanocrystals with extended pH and ionic stability. J. Mater. Chem., 2008, 18, $4949-4958$.

3. Semple, J. E., Sullivan, B., Vojkovsky, T., Still, K. N.; Synthesis and facile end-group quantification of functionalized PEG azides. J. Polym. Sci. A., 2016, 54, 2888 - 2895.

4. Mei, B. C.; Susumu, K., Medintz, I. L., Mattoussi, H.; Polyethylene glycol-based bidentate ligands to enhance quantum dot and gold nanoparticle stability in biological media. Nat. Protoc., 2009, 4, 3, $412-423$.

5. Susumu, K., Mei, B. C., Mattoussi, H.; Multifunctional ligands based on dihydrolipoic acid and polyethylene glycol to promote biocompatibility of quantum dots. Nat. Protoc., 2009, 4, 3, $424-436$.

6. Du, L.; Wang, W.; Zhang, C.; Jin, Z.; Palui, G.; Mattoussi, H. A Versatile Coordinating Ligand for Coating Semiconductor, Metal, and Metal Oxide Nanocrystals. Chem. Mater. 2018, 30 (20), 7269-7279.

7. Frens, G.; Controlled Nucleation for the Regulation of the Particle Size in Monodisperse Gold Suspensions. Nature, Phys. Sci. 1973, 241, 20-22.

8. Grabolle, M.; Spieles, M; Lesnyak, V.; Gaponik, N.; Eychmuller, A.; Resch-Genger, U.; Determination of the Fluorescence Quantum Yield of Quantum Dots: Suitable Procedures and Achievable Uncertainties. Anal. Chem., 2009, 81, 6285 - 6294. 